\title{
Multiple Working Mode Based Door Opening Control And Fault Detection of Mobile Modular And Reconfigurable Robot
}

\author{
by \\ Saleh Ahmad Ali \\ Master of Science in Engineering, Lakehead University, 2008
}

\author{
A dissertation \\ presented to Ryerson University \\ in partial fulfillment of the \\ requirements for the degree of \\ Doctor of Philosophy \\ in the Program of \\ Aerospace Engineering
}

Toronto, Ontario, Canada, 2013

(c)Saleh Ahmad Ali 2013 

I hereby declare that I am the sole author of this dissertation. This is a true copy of the dissertation, including any required final revisions, as accepted by my examiners.

I authorize Ryerson University to lend this dissertation to other institutions or individuals for the purpose of scholarly research

I further authorize Ryerson University to reproduce this dissertation by photocopying or by other means, in total or in part, at the request of other institutions or individuals for the purpose of scholarly research.

I understand that my dissertation may be made electronically available to the public. 

Multiple Working Mode Based Door Opening Control And Fault Detection of Mobile

Modular And Reconfigurable Robot

Doctor of Philosophy 2013

Saleh Ahmad Ali

Aerospace Engineering

Ryerson University

\begin{abstract}
The study in this thesis addresses the problem of opening a door with a modular and reconfigurable robot (MRR) mounted on a wheeled mobile robot platform. The foremost issue with door opening problems is the prevention of occurrence of large internal forces that arise due to position errors or imprecise modeling of the robot or its environment, i.e. the door parameters, specifically.

Unlike previous methods that relied on compliance control, making the control design rather complicated, this thesis presents a new concept that utilizes the multiple working modes of the MRR modules. The control design is significantly simplified by switching selected joints of the MRR to work in passive mode during door opening operation. As a result, the occurrence of large internal forces is prevented. Different control schemes are used for control of the joint modules in different working modes. For passive joint modules, a feedforward torque control approach is used to compensate the joint friction to ensure passive motion. For the active joint modules, a distributed control method, based on torque sensing, is used to facilitate the control of joint modules working under this mode. To enable autonomous door opening, an online
\end{abstract}


door parameter estimation algorithm is proposed on the basis of the least squares method; and a path planning algorithm is developed on the basis of Hermite cubic spline functions, with consideration of motion constraints of the mobile MRR. The theory is validated using simulations and experimental results, as presented herein.

A distributed fault detection scheme for MRR robots with joint torque sensing is also proposed in this thesis. The proposed scheme relies on filtering the joint torque command and comparing it with a filtered torque estimate that is derived from the nonlinear dynamic model of MRR with joint torque sensing. Common joint actuator faults are considered with fault detection being performed independently for each joint module. The proposed fault detection scheme for each module does not require motion states of any other module, making it an ideal modular approach for fault detection of modular robots. Experimental results have attested the effectiveness of the proposed fault detection scheme. 


\section{Acknowledgements}

I would like to thank all the wonderful people of Systems and Control Lab at Ryerson University. The completion of this thesis would have been impossible without the inspiration and continuous support of my supervisor, Dr. Guangjun Liu, who not just provided me ideal balance of encouragement, guidance, and opportunities, but also granted me an exceptional degree of freedom in pursuing my own ideas.

I would also like to thank Hongwei Zhang for insightful discussions, productive collaborations, late-night paper writing sessions, and our joint works on the MRR control design and implementation.

Next, I would like to thank Jahanzeb Tariq Khan for his friendship and for the perceptive discussions and support that he provided me during my Ph.D. studies.

Lastly, my deepest gratitude goes to my family for their unconditional support and love during every period of my life.

"If I have seen further, it is by standing on the shoulders of giants"

- Sir Isaac Newton, 1676.

Toronto, Ontario

Saleh Ahmad

Wednesday $8^{\text {th }}$ May, 2013 



\section{Dedication}

This thesis is dedicated to my family. 



\section{Contents}

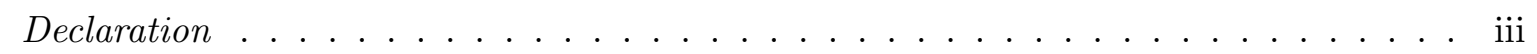

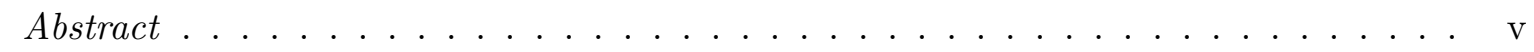

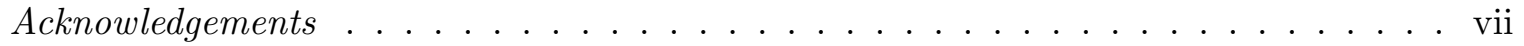

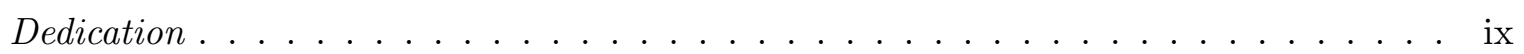

List of Tables . . . . . . . . . . . . . . . . . . . . x

List of Figures . . . . . . . . . . . . . . . . . . . . . . xvii

List of Appendices . . . . . . . . . . . . . . . . . . . xix

\begin{tabular}{lll}
\hline & Introduction & 1
\end{tabular}

1.1 Mobile Robot Manipulators Applications . . . . . . . . . . . . . . . . . . 3

1.1 .1 Service Robots . . . . . . . . . . . . . . . . . . . . . 3

1.1 .2 Security Applications $\ldots \ldots \ldots \ldots$. . . . . . . . . . . . . 3

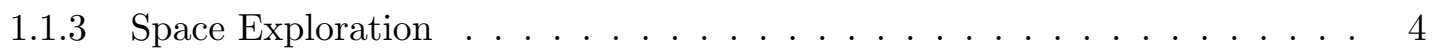

1.2 Thesis Contributions . . . . . . . . . . . . . . . . . . . . . . . 4

1.3 Outline of the Thesis $\ldots \ldots \ldots \ldots \ldots \ldots \ldots$

1.4 Publications . . . . . . . . . . . . . . . . . . . . . 6

$1.4 .1 \quad$ Journal Papers $\ldots \ldots \ldots \ldots \ldots \ldots$

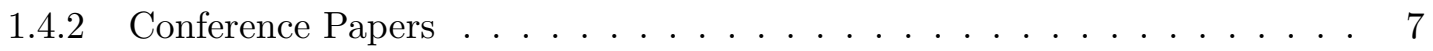

\begin{tabular}{|lll}
\hline 2 & System Diagnosis & 9
\end{tabular}

2.1 Introduction . . . . . . . . . . . . . . . . . . . . . . . . . . . . . . . 9

2.2 System Models $\ldots \ldots \ldots \ldots \ldots \ldots$

2.3 Fault Models $\ldots \ldots \ldots \ldots \ldots \ldots \ldots$. . . . . . . . . . . . . . . . . . 11

2.4 Fault Indicator Generation $\ldots \ldots \ldots \ldots \ldots \ldots$

2.5 Parameter Estimation . . . . . . . . . . . . . . . . . . . . . . . . . . . 12

2.5.1 Scalar Example with One Unknown Parameter . . . . . . . . . . . . . . . 14

2.5 .2 Parameter Drift $\ldots \ldots \ldots \ldots \ldots \ldots$

$2.6 \quad$ Fault Detection and Isolation $\ldots \ldots \ldots \ldots \ldots \ldots$ 
2.7 Robotic Joint Friction Models . . . . . . . . . . . . . . . . . . . . . . . . . . . . . . . 19

2.7 .1 Basics of Tribology $\ldots \ldots \ldots \ldots \ldots \ldots$

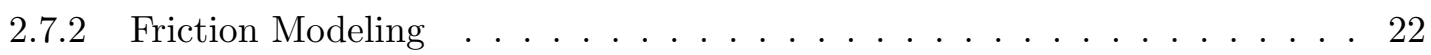

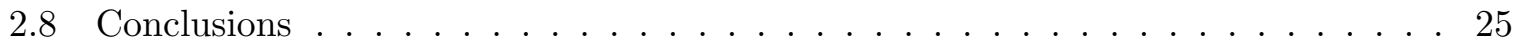

\begin{tabular}{|lll}
3 & Multiple Working Mode Control Design & 27
\end{tabular}

3.1 Introduction . . . . . . . . . . . . . . . . . . . . . . 27

$3.2 \quad$ Dynamic Model of MRRs with Joint Torque Sensing $\ldots \ldots \ldots \ldots . \ldots . \quad \ldots$

3.3 Passive Mode . . . . . . . . . . . . . . . . . . . . . . . . . . . . . . . . . . . . . . . 29

3.4 Active Mode . . . . . . . . . . . . . . . . . . . . . . . . . . . 31

$3.4 .1 \quad$ Control Design . . . . . . . . . . . . . . . . . . . . . . . 32

3.4 .2 Active Working Mode Experimental Results . . . . . . . . . . . . . 36

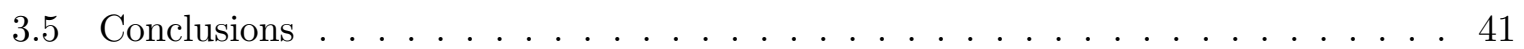

$4 \quad$ Multiple Working Mode Control of Door Opening with Mobile MRR 43

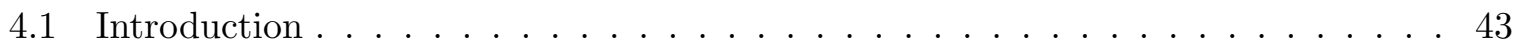

4.2 Related Work . . . . . . . . . . . . . . . . . . . . . . . . . . . 44

4.3 Problem Formulation . . . . . . . . . . . . . . . . . . . . . . . . . 46

4.4 Description of the Mobile MRR . . . . . . . . . . . . . . . . 47

$4.4 .1 \quad$ Modular and Reconfigurable Robot. . . . . . . . . . . . . . . 47

4.4 .2 Locomotion Base . . . . . . . . . . . . . . . . . . . . . . . . 48

4.5 Door Opening . . . . . . . . . . . . . . . . . . . . . . . 48

$4.5 .1 \quad$ Door Opening Method $\ldots \ldots \ldots \ldots$

4.5 .2 Door Parameters Estimation $\ldots \ldots \ldots \ldots \ldots$

4.5 .3 Path Planning . . . . . . . . . . . . . . . . . . 54

4.6 Simulation and Experiment Results . . . . . . . . . . . . . . . . . . 57

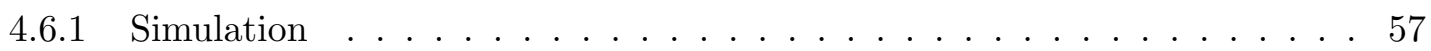

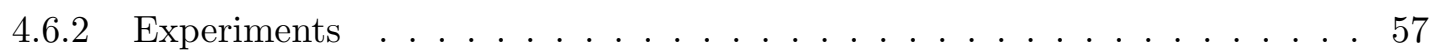

4.7 Conclusions $\ldots \ldots \ldots \ldots \ldots \ldots \ldots$

5 Distributed Fault Detection for Modular and Reconfigurable Robots $\quad 65$

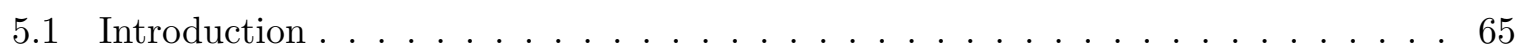

5.2 MRR Dynamic Model with Consideration of Faults . . . . . . . . . . . . 67

5.3 Prediction Error Based Fault Detection $\ldots \ldots \ldots$

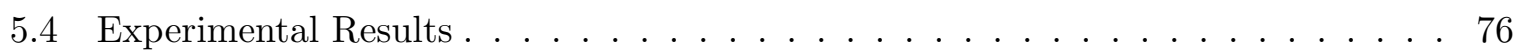

5.5 Conclusions $\ldots \ldots \ldots \ldots \ldots \ldots$ 
$\begin{array}{lll}6 & \text { Conclusions and Future Research } & 91\end{array}$

6.1 Conclusions . . . . . . . . . . . . . . . . . . . . . . . . . . . 91

$6.1 .1 \quad$ Strengths of the Proposed Approaches . . . . . . . . . . . . . . . . . 92

6.1 .2 Limitations of the Proposed Approaches . . . . . . . . . . . . . . . 93

6.2 Future Work . . . . . . . . . . . . . . . . . . . . . . . 93

\begin{tabular}{ll}
\hline References & 105
\end{tabular} 



\section{List of Tables}

$3.1 \quad$ External torque for MRR joint in passive mode. . . . . . . . . . . . . . . . . . 31

3.2 Physical parameters of the 3 -DOF MRR. . . . . . . . . . . . . . . . . 41

5.1 Physical parameters of the two-link MRR robot $\ldots \ldots \ldots$. . . . . . . 76

$5.2 \quad$ Delay times between the actuator faults occurrence and the fault detection. . . . 80 



\section{List of Figures}

1.1 The DESIRE robot; reprinted from $($ Prassler 2013). . . . . . . . . . . . . . . . 3

1.2 NASA's Mars rover Curiosity; reprinted from (Greicius 2012). . . . . . . . . . . . 4

2.1 Block diagram of a general system . . . . . . . . . . . . . . . . . . 10

2.2 Block diagram shows the implementation of the scalar adaptive, (2.8). . . . . . 15

2.3 Structure of model-based FDI system. . . . . . . . . . . . . . . . . . . . 19

2.4 Friction curve and the lubrication regimes. . . . . . . . . . . . . . . . . . 21

2.5 LuGre model simulation with different acceleration. . . . . . . . . . . . . . . . . 24

3.1 Schematic diagram of a joint module. . . . . . . . . . . . . . . . 28

3.2 Plots of the friction model shows the constant part of the friction. . . . . . . . . 30

3.3 3-DOF modular and reconfigurable robot. . . . . . . . . . . . . . . 36

3.4 Position tracking for the 3 -DOF MRR. . . . . . . . . . . . . . . . . . . 37

3.5 Position tracking errors for the 3-DOF MRR. . . . . . . . . . . . . . . . . 38

3.6 Torque sensor readings for the 3 -DOF MRR. . . . . . . . . . . . . . . . . . . . 39

3.7 Torque commands for the 3 -DOF MRR. . . . . . . . . . . . . . . . . 40

$4.1 \quad$ A planar model of door opening. . . . . . . . . . . . . . . . . . 47

4.2 5-DOF Mobile modular and reconfigurable robot. . . . . . . . . . . . . . . . . . 48

4.3 Comparison between traditional and proposed door opening method. . . . . . . . 51

4.4 Initial configuration of the mobile MRR. . . . . . . . . . . . . . . . . 52

4.5 Trajectory of the mobile base and the margin of allowed motion. . . . . . . . . . 57

4.6 Simulation results for $2 \mathrm{~cm}$ position error. . . . . . . . . . . . . . . . . . 58

4.7 Simulation results for $10 \mathrm{~cm}$ position error. . . . . . . . . . . . . . . . . 59

4.8 Schematic diagram of the door simulator. . . . . . . . . . . . . . . 60

4.9 Pictures of the mobile MRR pulling door open. ～. . . . . . . . . . . . . . . 61

4.10 Force measurements at the MRR end-effector. . . . . . . . . . . . . . . . . . 62

4.11 DAUJ pitch and yaw positions and torques. . . . . . . . . . . . . . . . . 62 
4.12 Torque sensor readings and joint angle of the MRR joint 1. . . . . . . . . . 62

4.13 End-effector forces with $2^{o}$ heading angle error. . . . . . . . . . . . . . 63

4.14 DAUJ angles and torques with $2^{\circ}$ heading angle error. . . . . . . . . . . . . 63

4.15 Joint 1 angle and torque sensor readings with $2^{\circ}$ heading angle error. . . . . . . . 64

5.1 Block diagram of distributed fault detection of one MRR joint module. . . . . . . 72

5.2 Two-link MRR robot. . . . . . . . . . . . . . . . . . 77

5.3 Fault free with nominal values, joint 1. . . . . . . . . . . . . . . . 79

5.4 Fault free, joint $1 . \ldots \ldots \ldots \ldots \ldots$. . . . . . . . . . . . . . . . . . .

5.5 Free-swinging actuator fault, joint $1 . \ldots \ldots \ldots \ldots$. . . . . . . . . . . . . . . . . . . . .

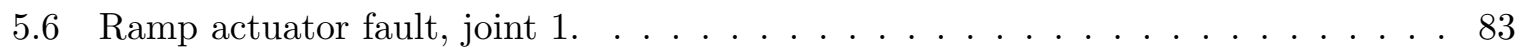

5.7 Saturated actuator fault, joint $1 . \ldots \ldots \ldots \ldots \ldots$

5.8 Fault free, joint $2 . \ldots \ldots \ldots \ldots \ldots \ldots \ldots$

5.9 Free-swinging actuator fault, joint $2 . \ldots \ldots \ldots \ldots$

5.10 Ramp actuator fault, joint $2 . \ldots \ldots \ldots \ldots$. . . . . . . . . 87

5.11 Saturated actuator fault, joint $2 . \ldots \ldots \ldots$. . . . . . . . 88

1.1 Example of two cubic splines. . . . . . . . . . . . . . . . 96 


\section{List of Appendices}

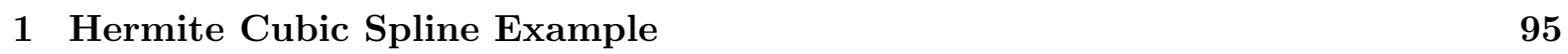

\begin{tabular}{|lll}
2 & Selection of the Upper Bound for the Dead-zone Residual Function & 97
\end{tabular} 



\section{Chapter 1}

\section{Introduction}

'Mobile manipulator' is nowadays a widespread term used to refer to robot systems built using a robotic arm that is mounted on a mobile platform. Such systems amalgamate the advantages of mobile platforms and robotic arms while reducing their individual drawbacks. For instance, an arm offers several operational functionalities and the mobile platform extends the workspace of the arm, which ultimately extends the reach of operational functionalities. Mobile robot manipulators promise to be the next frontier in robotics. Even though the need of fixed robots will remain perpetual in manufacturing sector, augmenting robot manipulators with mobile robots promises additional flexibility to end-users in new applications. Examples of such applications include personal assistance, medical and surgical uses, security, warehouse and distribution applications, as well as ocean and space exploration. The ability of one mobile robot to serve several locations and perform a greatly expanded range of tasks is greatly appealing for specialized applications.

Recently, mobile robots have found their way into other non-industrial applications, a success owed to the reduced cost of deployment and ownership associated with mobile robots. The current generation of mobile robots is serving hospitals, laboratories, and some offices where they replace skilled labor for mundane transportation tasks. Mobility is already the norm in service applications and this sector is primed for tremendous growth. Service robotics is expected to overtake the industrial robots sector in a matter of a few years. However, mobile robots are capable of performing only ordinary transportation tasks. To be able to carry out most of the other tasks, like flitching things, rescue applications, or home service applications, mobile robots have to be combined with robotic $\operatorname{arm}(\mathrm{s})$. At the moment, mobile manipulation topic is being focused upon significantly in development and research environments. Very few mobile manipulators, mostly teleoperated, are used scantly here and there like in space exploration, military operations, home-care and health-care. Many challenges and problems associated with 
autonomy are hindering the growth of mobile robot manipulators.

In the mobile manipulators' literature, traditional manipulators have been integrated with mobile platforms; however, traditional manipulators are position controlled with a fixed configuration and joints working in a single active mode. While traditional manipulators have been very successful at manipulating in controlled environments like a factory, they have performed sophisticated manipulation tasks in uncontrolled environments only when operated by a human. In addition, when integrated with mobile robots, they substantially limit the application potential of mobile manipulators. Within controlled environments, the world can be adapted to the capabilities of the robot. In uncontrolled environments, the robot has to adapt to the world consisting of only partially known objects and tasks, and real-time constraints. There are still many challenges to develop mobile manipulators for working in such uncontrolled environments or human environments. A typical example is that, opening a door is still a difficult task for robots.

This research aims to tackle such challenges by developing a mobile modular and reconfigurable robot that supports multiple working modes and utilizes this capability to find solutions to the problems of mobile manipulation in uncontrolled environments. A modular and reconfigurable robot can be defined as a 'robotic system constructed from a set of building blocks that are a collection of individual link and joint components'. By changing the configuration of these building blocks, a wide variety of specialized robots can be constructed. The development of a modular and reconfigurable robot (MRR) consisting of modular rotary joints is a part of ongoing research at our Systems and Control Lab. A unique feature of the locally developed MRR robotic joints is the implementation of both active and passive working modes on the same MRR modules. The work presented in this thesis utilizes this feature in solving problems that arise when working in uncontrolled environments, specifically the door opening problem.

In addition, in some applications, mobile manipulators are expected to operate in unknown environments where human intervention is not always possible and can be very expensive or even dangerous. It is therefore essential for the mobile MRR to independently detect and isolate internal failures and utilize the remaining functional capabilities to overcome the limitations forced by the failures.

The scope of this thesis is two folds. The first part addresses a key challenge in almost all mobile manipulator applications: the door opening problem. The second part of this thesis is dedicated to the design of distrusted fault detection scheme for modular and reconfigurable robots. 


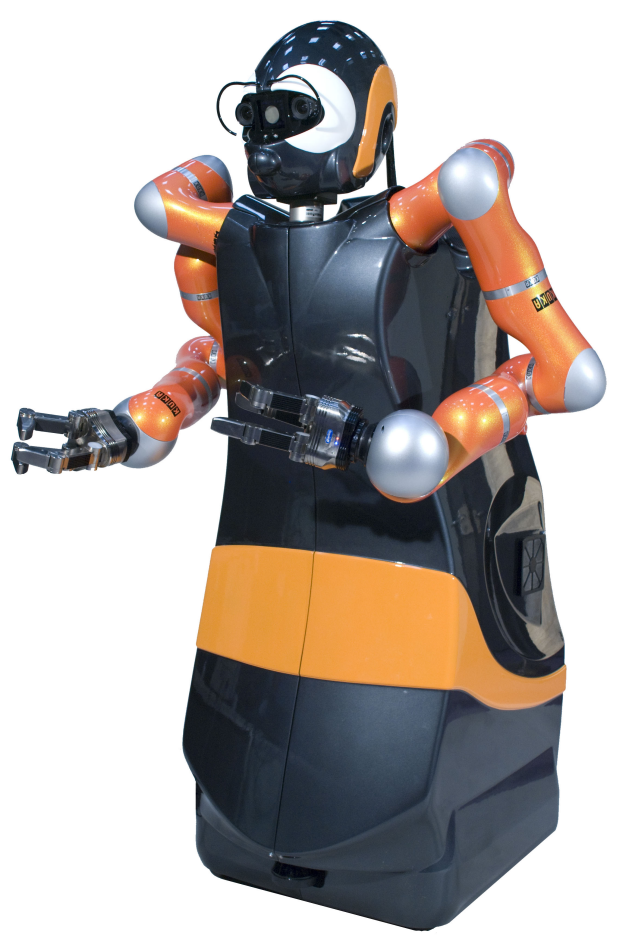

Figure 1.1: The DESIRE robot; reprinted from (Prassler 2013).

\section{$1.1 \quad$ Mobile Robot Manipulators Applications}

\subsubsection{Service Robots}

Service-robotics research has seen a rapid growth in recent years as robot control, programming, hardware and sensors mature. Service robots might provide day-to-day support in homes, doing laundry or dishes, assist in the care of elderly, or act as a caretaker for individuals within a home. One example of service robot is the DESIRE robot shown in Fig. 1.1. DESIRE is German service-robot initiative project, which was funded by the German Ministry of Education and Research. The development of a platform that can serve as a reference for daily use householdservice robots was the goal of the project.

\subsubsection{Security Applications}

Mobile robots may provide access to areas deemed dangerous to human beings such as nuclear power plants containing levels of radiation, especially during disaster times or potentially dis- 


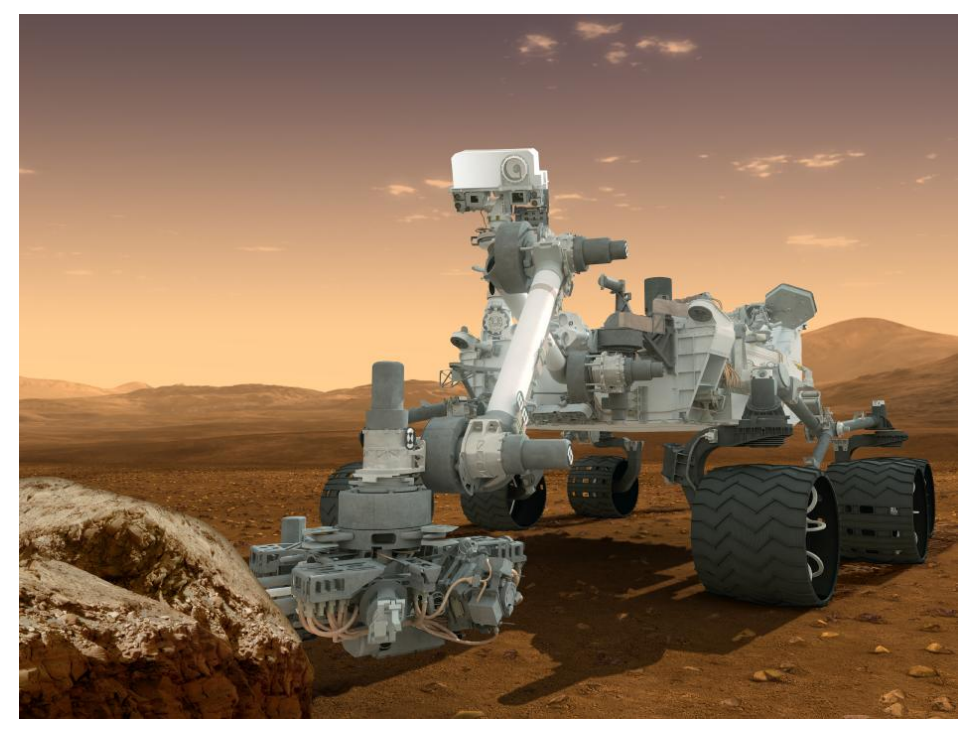

Figure 1.2: NASA's Mars rover Curiosity; reprinted from (Greicius 2012).

astrous times. Domains like monitoring, security and patrolling have seen significant expansion of applications of mobile robotics. Patrolling applications provide users with the ability to monitor intrusion on vacant properties or warehouse spaces. It also facilitates the monitoring of thermal and other environmental conditions. This additional ability is due to the reliability and low costs attributed to autonomous vehicle patrol capabilities.

\subsubsection{Space Exploration}

The rising interest in space exploration missions has also spurred growth in mobile robot manipulators, a few examples of which are the Spirit, Opportunity, and the Curiosity rover. The Curiosity shown in Fig. 1.2 is the most recent Mars exploration project, a part of NASA's Mars Science Laboratory mission, the aim of which is to explore Gale Crater on Mars. The main scientific goals of this car-sized robotic rover are to investigate the possibility of existence of human life on the red planet, determine the role of water, and to study the climate and geology of Mars. The mission will also pave the way for human exploration.

\subsection{Thesis Contributions}

As mentioned previously, the research work presented in this thesis is concentrated on two objectives: development of door opening control strategy, and fault detection algorithm for MRRs with joint torque sensing. More highlights of thesis contributions are as follows: 
- An autonomous control strategy for door opening with mobile MRR based on the framework of the multiple working mode control

- A novel door opening strategy

- The proposed door opening method does not require prior knowledge about the door parameters

- Door parameter estimation algorithm

- Path planning method for the mobile MRR

- Design of decentralized fault detection for modular and reconfigurable robots with joint torque sensing

- The proposed distributed fault detection scheme does not require acceleration measurements

- The proposed fault detection of each joint module is independent of the motion states of other joints

\subsection{Outline of the Thesis}

The thesis is divided into six chapters, and a brief outline of the next five chapters is as follows:

Chapter 2 The second chapter is meant to introduce the basics of system diagnosis. The material covered in this chapter tends to focus on reviewing various methods of system diagnosis, with special emphasis put on analytical fault detection methods because these methods form the roots of the proposed fault detection algorithm in Chapter 5 . The chapter also presents an overview of friction in robotic joints from both empirical and phenomenological perspectives. Both static and dynamic friction models are described. These models have their due importance not just in the design and validation of the proposed distributed fault detection algorithm but also in the design of multiple mode control of the MRR.

Chapter 3 The multiple working mode control design concepts are described in this chapter. The first part of this chapter discusses the dynamic model, utilized in the active working mode control design, of modular and reconfigurable robots with joint torque sensing. The later subsections describe, in detail, the passive and active working modes used to devise the door opening method. Related experimental results for both working modes are also documented in this chapter. 
Chapter 4 One of the two primary contributions of this research work, the proposed door opening method by a mobile modular and reconfigurable robot, is detailed in this chapter. The chapter opens with the foremost requirement of any research work, literature review, which in this case covers the topic of door opening with mobile robot manipulators. The subsequent sections of this chapter detail the formulation of door opening task using the mobile MRR. The theoretically proposed door opening method was endorsed through simulations and experimental results, both of which are also documented in the later subsections of this chapter. Concluding remarks close the chapter.

Chapter 5 The purpose of this chapter is twofold: i) describe the proposed distributed fault detection scheme for modular and reconfigurable robots, and ii) to show how the proposed method can be applied to detect actuator faults. A brief literature review of previous research works on fault detection of robot manipulators commences this chapter. The following section describes the dynamic model of MRR with joint torque sensing that may undergo actuator fault. The proposed distributed prediction error based fault detection approach is detailed in the third section. Experimental results attesting the theoretical contributions along with the concluding remarks are the subject of last two sections of this chapter.

Chapter 6 The last chapter summarizes the contributions and achievements of this thesis, hints at directions for possible future research, and discusses the open challenges related to the proposed door opening method and fault detection scheme.

\subsection{Publications}

\subsubsection{Journal Papers}

1. S. Ahmad, H. Zhang, and G. Liu, "Multiple Working Mode Control of Door Opening with a Mobile Modular and Reconfigurable Robot", IEEE/ASME Trans. on Mech., vol. 18, no. 3, pp. 833-844, Jun., 2013.

2. S. Ahmad, H. Zhang, and G. Liu, "Distributed Fault Detection for Modular and Reconfigurable Robots with Joint Torque Sensing: A Prediction Error Based Approach", Revision submitted to Mechatronics, Jan., 2013.

3. H. Zhang, S. Ahmad, and G. Liu, "Modeling and Analysis of Compliance and Hysteresis in Harmonic Drives", Revision submitted to IEEE/ASME Trans. on Mech., Jan., 2013. 


\subsubsection{Conference Papers}

1. G. Liu, S. Ahmad, and L. Ren, "Hybrid Control of Door-opening by Modular Reconfigurable Robots", in Proc. IEEE/RSJ Int. Conf. Intell. Robots Syst., St. Louis, MO, Oct. 11-15, 2009, pp. 1480-1485.

2. S. Ahmad, and G. Liu, "A Door Opening Method by Modular Re-configurable Robot with Joints Working on Passive and Active Modes", in Proc. IEEE Int. Conf. Robot. Autom., Anchorage, AK, May 3-8, 2010, pp. 1480-1485.

3. H. Zhang, S. Ahmad, G. Liu, "Torque Estimation of Robot Joint with Harmonic Drive Transmission", in Proc. IEEE Int. Conf. Robot. Autom., Karlsruhe, Germany, May 6-10, 2013, Accepted for publication.

4. S. Ahmad, G. Liu, "Model-based Fault Detection of Modular and Reconfigurable Robots with Joint Torque Sensing", in Proc. IEEE/ASME Int. Conf. Adv. Intell. Mechatron., Wollongong, Australia, July 9-12, 2013, Accepted for publication. 



\section{Chapter 2}

\section{System Diagnosis}

\section{$2.1 \quad$ Introduction}

Diagnosis is a multidisciplinary science covering a wide spectrum of applications across different fields. Owing to its relevance in different fields of applications, extensive literature is available on the system diagnosis topic, some examples of which are (Zeigler 1992), (Basseville and Nikiforov 1993), (Liu et al. 2000), (Gustafsson 2000), (Yongli et al. 2006), and (Bishop 2007). Because of its diversity, it is challenging to find a standard presentation and categorization code for different methods and solutions. An important contribution towards a common framework was presented in a special issue of the IEEE Transactions on Systems, Man and Cybernetics journal, in 2004 (Biswas et al.). System diagnosis was considered as an area of research for two communities primarily, which are:

- The Fault Detection and Isolation (FDI) community, a community that borrows concepts largely from control theory and statistical decision making, and

- The Diagnosis (DX) community, having roots in computer science and artificial intelligence.

However, diagnosis is not a research topic limited to the above mentioned fields only. Professionals of other fields focus on system diagnosis as well, but have their own terminology to refer to it. For example, the mechanical engineers call it 'condition monitoring' while the computer scientists specializing in machine learning deal within the context of classification problems. It is beyond the scope of this thesis to provide a comprehensive overview of the various diagnosing solutions and terminology but an interested reader may refer to the work reported in (Venkatasubramanian et al. 2003, Nandi et al. 2005). It is aimed that the contents of this 
chapter introduce the problems to a reader and facilitate the contextualization of the methods developed in this work.

\section{$2.2 \quad$ System Models}

A thorough understanding of the system behavior and its dependencies on the faults is critical for choosing the appropriate diagnosing algorithm. Models may be used for this purpose. For the purposes of this research work, a system is a mechanism that generates data with which a diagnosis can be performed. Consider the block diagram shown in Fig. 2.1, where the system represents the mechanism that takes in two inputs, deterministic input ' $z$ ' and random inputs ' $v$ ' (e.g. noise), and outputs measurable data $y$. ' $v$ ' inputs are assumed to be unknown, while $z$ could have partially known components. The set of deterministic inputs, $z$, is further broken down into three distinct categories: $u, d$ and $f$ with $u$ being known input (e.g. control inputs), $d$ being unknown and uninteresting input (e.g. disturbances), and $f$ being unknown but an input of interest (e.g. faults). The signals $y$ and $u$ are known and critical for the design of diagnosis solutions. Inputs $v, d$ and $f$ all have an aggregate effect on $y$ and $u$, and segregating the effects of these inputs is important to determine their individual effects on the output. Generally, a system model is a map from $z$ and $v$ to $y$, i.e.

$$
y=g(z, v)
$$

When this map is parameterized with parameters $\theta$, it is called a model structure, $M$,

$$
M: y=g(z, v, \theta)
$$

A particular parameter choice, $\bar{\theta}$, leads to a model instance, $M(\bar{\theta})$, of the model structure $M$. A system model structure can be obtained through either careful modeling that is based on physical principles and well-established relations or by determining the model structure directly from available data. For the earlier case, the parameters in such models are related to some physical meaning. If there are known parameters, the system model may either be called white

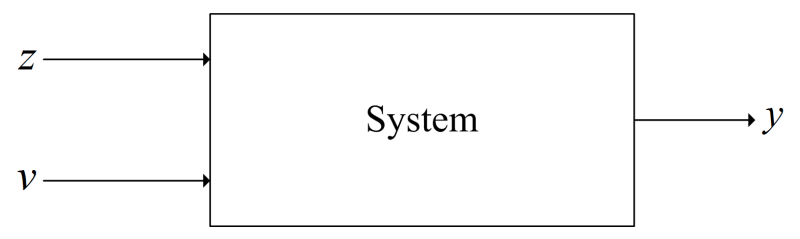

Figure 2.1: Block diagram of a general system 
box or gray box; white if all parameters are known, or gray box if some parameters are known. In the case of gray box models, the unknown parameters must be determined, e.g. by using an identification procedure. In the latter case, the resulting model is called black box and its parameters have no obvious physical interpretation. System models that are based on physical principles have larger generalization capacity comparing to those obtained from data.

Remark 1 The term generalization capacity refers to the degree to which a model can simulate the actual behavior of a system. Generalization capacity is an important characteristic that typically has larger values for models derived from physical principles than black box models.

\subsection{Fault Models}

Fault modeling is of special importance. The chosen fault model must mock up the physical effects of a fault and the way it is reflected in the available data. Faults can be categorized either by their time behavior or by the manner they affect the system. With respect to the time behavior, a fault might be:

- Abrupt, a fault that affect the system abruptly, stepwise. Abrupt fault may represent bias in the monitored signal.

- Incipient, a fault that develop gradually with time. Incipient faults are modeled by using ramp signals. Incipient fault may represent drift of the monitored signal.

- Intermittent, a fault that affect the system with interruptions. It could be described by combination of impulses with different amplitudes.

Depending upon the kind of affect a fault may has on the system, it may be:

- Additive, i.e. faults that are effectively added to the system's inputs or outputs. Additive faults influences a variable of the system by an addition of the fault itself. They may represent, e.g., offsets of sensors.

- Multiplicative, i.e. faults acting on a parameter of the system. For example, changing a parameter $\theta$ of the model structure $M$.

- Structural, i.e. faults introducing new governing terms to the describing equations of the system. For example, changing the model structure $M$.

Additive faults and multiplicative faults are typically used to model sensor faults and system faults respectively. 


\subsection{Fault Indicator Generation}

Fault indicator generation task has the purpose of signalling a fault's occurrence by means of a user designed algorithm that operates on dataset $(u, y)$. In other words, it is a sort of a virtual sensor that outputs a signal only upon perceiving a fault. In case if there is any available information about the unknowns $(v, d, f)$, it can be used to design fault indicator. The system model structure $M$ is a key indicative of system behavior and as such its importance in designing fault indicators is unquestionable. Two fault indication methods are presented below, both of which are based on the assumption that the actual model instance generating the data is given by $M\left(\theta_{0}\right)$.

Output observer: In this case, the model instance, $M\left(\theta_{0}\right)$, is assumed to be known and is used to reconstruct the output from the data $(u, y)$, thereby creating an analytical redundancy $\hat{y}\left(\theta_{0}\right)$ that provides a fault indication given by $e\left(\theta_{0}\right)=y-\hat{y}\left(\theta_{0}\right)$, also known as residual.

It should be observed that residual indicates a non-zero difference between the observations and the reference behavioral mode related to $\theta_{0}$. In other words, the residual $e\left(\theta_{0}\right)$ can be considered as a test quantity which for nominal parameters, $\theta_{0}$, indicates deviation from a faultless reference behavioral mode. Additional test quantities are also obtainable by further processing the residuals $e\left(\theta_{0}\right)$ if multiple reference modes are available.

Parameter estimation: The parameter $\theta_{0}$ has its estimate $\hat{\theta}$ obtained through parameter estimation techniques that rely on measured data $(u, y)$ and knowledge of model structure $M$. Parameter estimation technique is a natural choice for multiplicative faults. In case if external knowledge about $\theta_{0}$ is available, a test quantity can be defined as $\hat{\theta}-\theta_{0}$ to indicate deviations from the reference behavior related to $\theta_{0}$.

$\hat{\theta}$ can also be used to provide an output estimate $\hat{y}(\hat{\theta})$ and subsequently the residual $e(\hat{\theta})=$ $y-\hat{y}(\hat{\theta})$ since both are functions of $\hat{\theta}$. Residual $e(\hat{\theta})$ serves as a test quantity for diagnosing structural faults. Additional test quantities can also be defined provided that other reference behavioral modes are known just like in the output observer case.

Remark 2 Whereas, the parameter estimation techniques are a natural choice for multiplicative and structural faults, the output observers are more suitable for additive faults. Nevertheless, these methods can be used interchangeably, (Isermann 2006).

\subsection{Parameter Estimation}

Parameter estimation is the technique of estimating the unknown parameters of a known model structure by using measured data. The solutions provided by the parameter estimation algo- 
rithm depend upon the model structure and the necessity for a real-time method Gustafsson 2000). To illustrate the concepts of parameter estimations, the prediction error method, adapted from (Ioannou and Tsakalis 1986), is described next. Let us consider systems described by ordinary differential equations of the form:

$$
\dot{x}=f(t, x) \quad x\left(t_{0}\right)=x_{0}
$$

where $x \in \Re^{n}, f: J \times B(r) \mapsto R, J=\left[t_{0}, \infty\right)$ and $B(r)=\left\{x \in \Re^{n}|| x \mid<r\right\}$. We assume that $f$ is of such nature that for every $x_{0} \in B(r)$ and every $t_{0} \in \Re^{+}, 2.3$ possesses one and only one solution $x\left(t ; t_{0}, x_{0}\right)$.

Definition 1 A continuous function $\varphi:[0, r] \mapsto \Re^{+}$(or a continuous function $\varphi:[0, \infty) \mapsto$ $\Re^{+}$) is said to belong to the class $\mathcal{K}$, i.e., $\varphi \in \mathcal{K}$ if

i) $\varphi(0)=0$

ii) $\varphi$ is strictly increasing on $[0, r]$ or on $(0, \infty)$.

Definition 2 A continuous function $\varphi:[0, \infty) \mapsto \Re^{+}$is said to belong to the class $\mathcal{K} \mathcal{R}$, i.e., $\varphi \in \mathcal{K} \mathcal{R}$ if,

i) $\varphi(0)=0$

ii) $\varphi$ is strictly increasing on $[0, \infty)$

iii) $\lim _{r \rightarrow \infty} \varphi(r)=\infty$.

Theorem 1 Assume that 2.3 possesses unique solutions for all $x_{0} \in \Re^{n}$. If there exists a function $V(t, x)$ defined on $|x| \geq R$ (where $R$ may be large) and $t \in[0, \infty)$ with continuous first-order partial derivatives with respect to $x, t$ and if there exist $\varphi_{1}, \varphi_{2} \in \mathcal{K} \mathcal{R}$ such that:

i) $\varphi_{1}(|x|) \leq V(t, x) \leq \varphi_{2}(|x|)$

ii) $\dot{V}(t, x) \leq 0$

for all $|x| \geq R$ and $t \in[0, \infty)$, then, the solutions of 2.3 are $u . b$. If in addition there exists $\varphi_{3} \in \mathcal{K}$ defied on $[0, \infty)$ and

iii) $\dot{V}(t, x) \leq-\varphi_{3}(|x|)$ for all $|x| \geq R$ and $t \in[0, \infty)$

then, the solutions of 2.3 are u.u.b.

Lemma 1 if $f, \dot{f} \in \mathcal{L}_{\infty}$ and $f$ belongs to $\mathcal{L}_{p}$ for some $p \in[1: \infty)$, then $f(t) \rightarrow 0$ as $t \rightarrow \infty$.

Lemma 2 (Barbălate's Lemma) if $\lim _{t \rightarrow \infty} \int_{0}^{t} f(\tau) d \tau$ exists and finite, and $f(t)$ is uniformly continuous function, then $\lim _{t \rightarrow \infty} f(t) \rightarrow 0$. 


\subsubsection{Scalar Example with One Unknown Parameter}

Consider the system described by the following algebraic equation:

$$
y(t)=\theta u(t)
$$

where $y(t)$ is the output, $u \in \mathcal{L}_{\infty}$ is the scalar input, and $\theta$ is an unknown parameter. With the assumption that $u(t), y(t)$ are measured, it is desired to obtain an estimate of $\theta$ at each time $t$. We are interested in online method to generate $\hat{\theta}(t)$. A differential equation, which depends on signals that are measured, whose solution is $\hat{\theta}(t)$ and its equilibrium state is $\theta_{e}=\theta$ is looked-for. The method for developing such a differential equation is described next.

Using $\hat{\theta}(t)$ as the estimate of $\theta(t)$ at time $t$, one can generate the estimated or predicted value $\hat{y}(t)$ of the output $y(t)$ as:

$$
\hat{y}(t)=\hat{\theta}(t) u(t)
$$

The estimation error $\mathbf{e}_{1}$, which reflects the parameter uncertainty, is formed as the discrepancy between $\hat{y}$ and $y$, i.e.,

$$
\mathbf{e}_{1}=y-\hat{y}=y-\hat{\theta} u(t)
$$

The prediction error, $\mathbf{e}_{1}$, is dependent on the parameter estimation error $\tilde{\theta} \triangleq \hat{\theta}-\theta$. This fact becomes clear if one substitute 2.4 into (2.6), i.e.,

$$
\mathbf{e}_{1}=\theta u(t)-\hat{\theta} u(t)=-\tilde{\theta} u(t)
$$

The differential equation that generate $\hat{\theta}(t)$ can be developed by minimizing various cost criteria of the prediction error $\mathbf{e}_{1}$ with respect to $\hat{\theta}$. For example, one can use the gradient or Newton's method to achieve this objective. For the purpose of illustration of this example, the following simple cost criterion is considered,

$$
J(\hat{\theta})=\frac{\mathbf{e}_{1}^{2}}{2}=\frac{(y-\hat{\theta} u)^{2}}{2}
$$

The function $J(\hat{\theta})$ is convex over $\Re$ which indicate that any local minimum of $J$ is also global and satisfies $\nabla J(\hat{\theta})=0$. One can solve $\nabla J(\hat{\theta})=-(y-\hat{\theta} u) u=0$ for $\hat{\theta}$ and use the gradient method to form the recursive scheme as:

$$
\dot{\hat{\theta}}=-\gamma \nabla J(\hat{\theta})=\gamma(y-\hat{\theta} u) u=\gamma \mathbf{e}_{1} u, \quad \hat{\theta}(0)=\hat{\theta}_{0}
$$

where $\gamma>0$ is a scaling constant, which is usually referred to as the adaptive gain. 


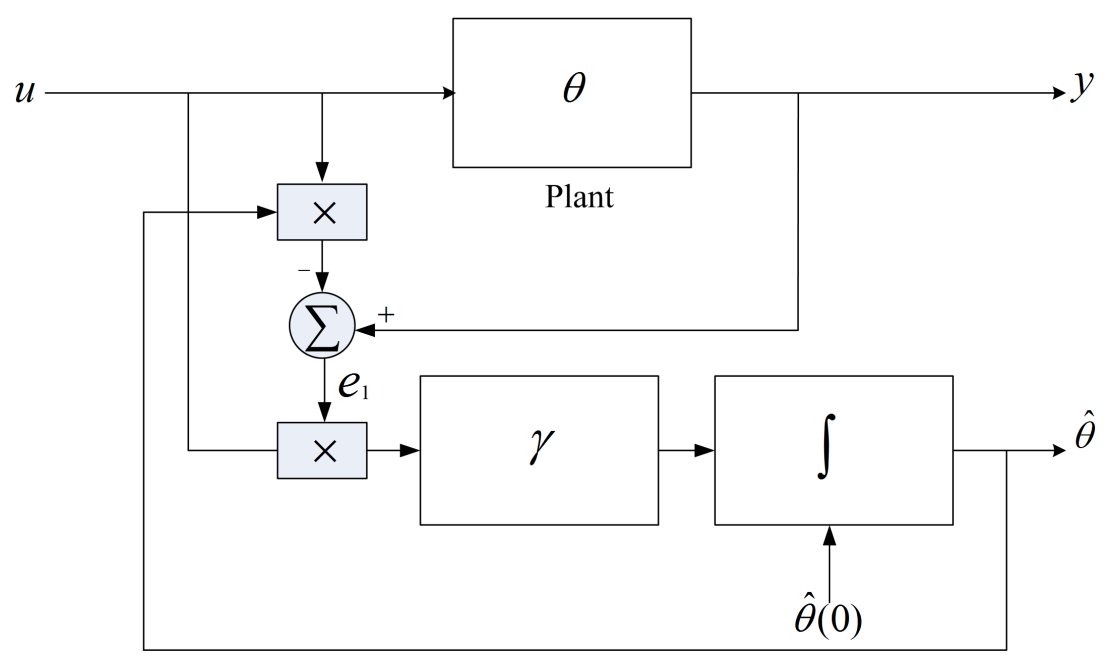

Figure 2.2: Block diagram shows the implementation of the scalar adaptive, (2.8).

The differential equation (2.8) is usually referred to as the update/adaptive law or the estimator. In this thesis, we refer to (2.8) as the adaptive law for updating $\hat{\theta}(t)$ or estimating $\theta$ online. Figure 2.2 shows the implementation of the adaptive given in (2.8). The stability properties of 2.8 are analyzed by rewriting the same equation in terms of the parameter error $(\tilde{\theta}=\hat{\theta}-\theta)$, as:

$$
\dot{\tilde{\theta}}=\dot{\hat{\theta}}-\dot{\theta}=\gamma \mathbf{e}_{1} u-\dot{\theta}
$$

Because $\mathbf{e}_{1}=\theta u-\hat{\theta} u=-\tilde{\theta} u$ and $\theta$ is constant, one has:

$$
\dot{\tilde{\theta}}=-\gamma u^{2} \tilde{\theta}, \quad \tilde{\theta}(0)=\hat{\theta}(0)-\theta
$$

It should be emphasized that 2.9 is used only for analysis. This equation cannot be used to generate $\hat{\theta}(t)$ because given an initial estimate $\hat{\theta}(0)$ of $\theta$, the initial value $\tilde{\theta}(0)=\hat{\theta}(0)-\theta$, which is required for implementing 2.9 is unknown due to the unknown $\theta$.

We can analyze (2.9) by choosing the following Lyapunov function:

$$
V(\tilde{\theta})=\frac{\tilde{\theta}^{2}}{2 \gamma}
$$

The time derivative of $V(\tilde{\theta})$ along the solution of 2.9 is given by:

$$
\dot{V}=\frac{\tilde{\theta}^{T} \dot{\tilde{\theta}}}{\gamma}
$$


After substitution of $\dot{\tilde{\theta}}$ from 2.9 , the last equation becomes:

$$
\dot{V}=-u^{2} \tilde{\theta}^{2}=-\mathbf{e}_{1}^{2} \leq 0
$$

This result implies that the equilibrium $\tilde{\theta}_{e}=0$ of 2.9$)$ is u.s.

Since no further information about $u(t)$ is assumed other than $u \in \mathcal{L}_{\infty}$, one cannot guarantee that $\dot{V}<0$ (e.g., take $u(t)=0$ ) and, therefore, cannot establish that $\tilde{\theta}_{e}=0$ is a.s. or e.s. One can, however, use the properties of $V, \dot{V}$ to establish convergence for the estimation error and other signals in (2.8). For example, because $V \geq 0$ is a nonincreasing function of time, the $\lim _{t \rightarrow \infty} V(\tilde{\theta}(t))=V_{\infty}$ exists. Therefore, from 2.10 one has:

$$
\int_{0}^{\infty} \mathbf{e}_{1}^{2}(\tau) d \tau=-\int_{0}^{\infty} \dot{V}(\tau) d \tau=V_{0}-V_{\infty}
$$

where $V_{0}=V(\tilde{\theta}(0))$, which implies that $\mathbf{e}_{1} \in \mathcal{L}_{2}$. From 2.8$)$ and $u \in \mathcal{L}_{\infty}$, it can be concluded that $\dot{\hat{\theta}} \in \mathcal{L}_{\infty} \cap \mathcal{L}_{2}$. It is well known that a square integrable function may not have a limit, let alone tend to zero with time, therefore, one cannot establish that $\mathbf{e}_{1}(t), \dot{\hat{\theta}}(t) \rightarrow 0$ as $t \rightarrow \infty$ without satisfying additional conditions. However, if one assume that $\dot{u} \in \mathcal{L}_{\infty}$, then it follows that $\dot{\mathbf{e}}_{1}=-\dot{\tilde{\theta}} u-\tilde{\theta} \dot{u} \in \mathcal{L}_{\infty}$, therefore, from Lemma 2 one has $\mathbf{e}_{1}(t) \rightarrow 0$ as $t \rightarrow \infty$, which is implied by $\mathbf{e}_{1} \in \mathcal{L}_{2}, \dot{\mathbf{e}}_{1} \in \mathcal{L}_{\infty}$. This, in turn, leads to:

$$
\lim _{t \rightarrow \infty} \dot{\tilde{\theta}}(t)=\lim _{t \rightarrow \infty} \dot{\hat{\theta}}(t)=0
$$

It can be concluded from this analysis that, for any $u, \dot{u} \in \mathcal{L}_{\infty}$, the adaptive law 2.8 guarantees that the estimated output $\hat{y}(t)$ converges to the actual output $y(t)$ and the speed of adaptation, i.e., the rate of change of the parameters $\dot{\hat{\theta}}$, decreases with time and converges to zero asymptotically. However this does not mean $\hat{\theta}(t)$ converges as $t \rightarrow \infty$.

\subsubsection{Parameter Drift}

Consider the same system describe in the example in subsection (2.5.1), but the system output is now corrupted by an unknown bounded disturbance $d(t)$, i.e.,

$$
y=\theta u+d
$$

The adaptive law for estimating $\theta$ that was derived in the previous example for $d(t)=0 \forall t \geq 0$ is given by:

$$
\dot{\hat{\theta}}=\gamma \mathbf{e}_{1} u, \mathbf{e}_{1}=y-\hat{\theta} u
$$


where $\gamma>0$ and $\hat{\theta}(t)$ is the online estimate of $\theta$. In the previous example, it was shown that for $d(t)=0$ and $u, \dot{u} \in \mathcal{L}_{\infty}, 2.12$ guarantees that $\hat{\theta}, \mathbf{e}_{1} \in \mathcal{L}_{\infty}$ and $\mathbf{e}_{1} \rightarrow 0$ as $t \rightarrow \infty$. Let us now analyze 2.12 when $d(t) \neq 0$.

Substituting for $\tilde{\theta} \triangleq \hat{\theta}-\theta$ into the prediction error equation, one obtains:

$$
\mathbf{e}_{1}=-\tilde{\theta} u+d
$$

and,

$$
\dot{\tilde{\theta}}=-\gamma u^{2} \tilde{\theta}+\gamma d u
$$

Now, we can analyze 2.13 by considering the following function:

$$
V(\tilde{\theta})=\frac{\tilde{\theta}^{2}}{2 \gamma}
$$

The time derivative of $V(\tilde{\theta})$ along the trajectory of 2.13 is:

$$
\begin{aligned}
\dot{V} & =-\tilde{\theta}^{2} u^{2}+d \tilde{\theta} u \\
& =-\frac{\tilde{\theta}^{2} u^{2}}{2}-\frac{1}{2}(\tilde{\theta} u-d)^{2}+\frac{d^{2}}{2}
\end{aligned}
$$

For the class of input that is considered here, i.e., $u \in \mathcal{L}_{\infty}$ one cannot conclude that $\tilde{\theta}$ is bounded from consideration of 2.14, 2.15), i.e., one cannot find a constant $V_{0}>0$ such that for $V>V_{0}, \dot{V} \leq 0$.

\subsection{Fault Detection and Isolation}

Fault detection and isolation (FDI) can be performed through either:

- Direct pattern recognition of sensor readings that can indicate a fault, or

- By analyzing the discrepancy between the sensor readings and expected values derived from the system model.

For the latter case, the fault detection algorithm declares a fault if the discrepancy or residual exceeds a certain threshold, after which the fault isolation algorithm subsequently categorizes the type of fault and its location in the system. 


\section{Model-based Fault Detection Approach}

Model-based FDI can be defined as the detection, isolation and characterization of faults on a system by means of methods which extract features from measured signals and use system mathematical models. Faults are therefore detected by setting fixed or variable thresholds on residual signals generated from the difference between actual measurements and their estimates obtained by using the system model. The attention received by the model-based fault detection algorithms has been on the rise for the past two decades. As such, a great variety of techniques that relies on using mathematical models of the system under investigation can be found in the literature. Model-based fault detection algorithms require accurate modeling of the behavior of the monitored system, and any discrepancy in that model form the actual system behavior may cause problems to the FDI scheme. As such, the modeling inaccuracy issue forms the core of research that has been carried out on this topic in recent years. Modeling uncertainty arises from the impossibility of obtaining complete knowledge and understanding of the monitored process, as well as from the presence of noises on process measurements.

Keeping in mind that one of the goals of this research work is to develop and design a model-based FDI algorithm for MRRs, the basic principles of model-based fault detection are introduced below. Multiple different residuals can be designed to individually detect faults occurring at various locations of a system. The analysis of each residual, upon exceeding of threshold, subsequently leads to fault isolation. A general block diagram of the model-based FDI system, comprising of two main stages that are residual generation and residual evaluation, is depicted in Fig. 2.3. The two main blocks of this structure, widely accepted by the fault diagnosis community, are described below:

- Residual generation block: this block generates residual signals, to indicate any fault occurrence, by using available inputs and outputs from the monitored system. It should normally be zero or its proximity under no fault condition, but distinguishably different from zero otherwise. It would imply that, under ideal conditions, the residual is characteristically independent of process inputs and outputs. The method of computing residuals is called residual generation, and is depicted in Fig. 2.3 .

- Residual evaluation: This block evaluates the likelihood of fault occurrence by examining the residuals and then applies decision rule to determine any faults occurrence. The residual evaluation block, shown in Fig. 2.3, may perform a simple threshold test on the instantaneous values or moving averages of the residuals, or it may consist of statistical methods, like generalized likelihood ratio testing or sequential probability ratio testing, for example. 


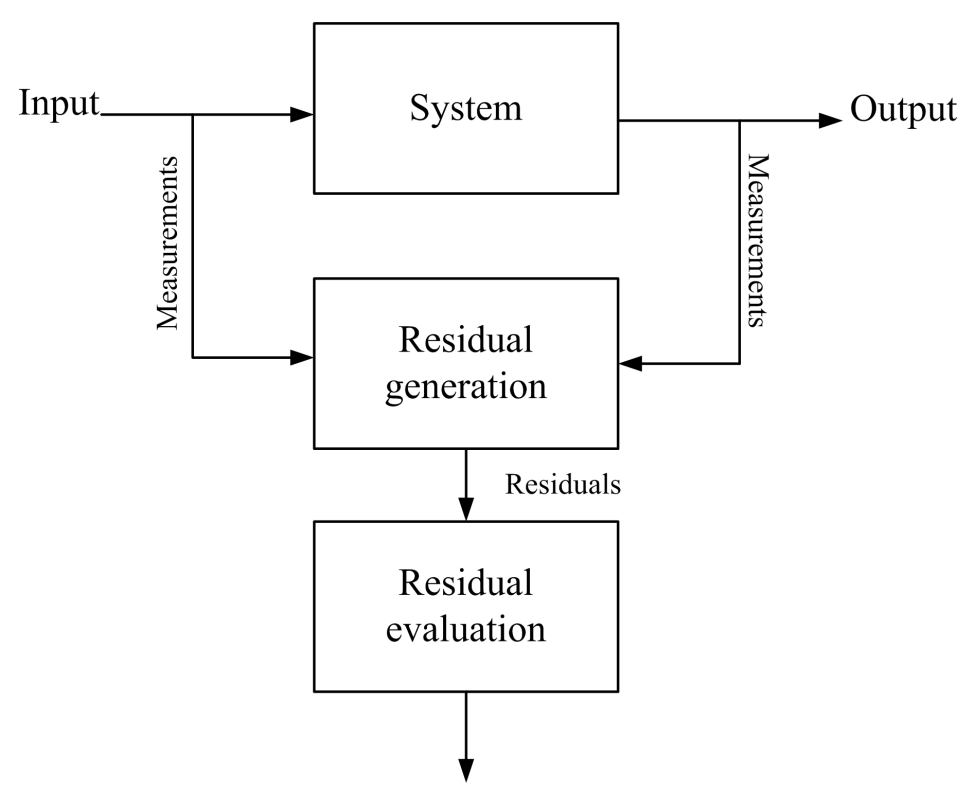

Fault Alarm

Figure 2.3: Structure of model-based FDI system.

Most of the work in the field of model-based FDI focuses on the residual generation problem, because well-designed residuals makes the decision making process easy. Fault detection schemes for MRRs can be devised by utilizing analytical redundancy techniques and exploiting the integrated torque sensor. In this work, the system's modularity will be exploited to derive a strategy dependent only on a single joint model by eliminating the need for the motion state of the other joint modules. The dynamic model for MRR with joint torque sensing is utilized in the fault detection method.

\subsection{Robotic Joint Friction Models}

Friction, defined as a tangential reaction force between two surfaces in contact, is a force that exists in all practical systems and mechanisms in varying magnitude. It results from complex interactions between contacting surfaces at microscopic level. There are different types of friction out of which the four most common ones are: dry friction 1 , viscous friction ${ }^{2}$, lubricated

\footnotetext{
${ }^{1}$ Dry friction is the resistance to relative lateral motion of two solid surfaces in contact.

${ }^{2}$ Viscous friction is the resistance of a fluid to flow. This resistance acts against the motion of any solid object through the fluid and also against motion of the fluid itself past stationary obstacles. Viscosity also acts internally on the fluid between slower and faster moving adjacent layers.
} 
friction 3 , and skin friction 4 . Describing friction from physical principles is challenging due to its complex nature and therefore, Tribology, the science of interacting surfaces in relative motion, is mostly based on empirical studies. Friction in robotic joints needs to be modeled for control and fault detection purposes because precise modeling of it plays a critical role to improve performance with respect to accuracy and control stability, see for e.g., (Mu et al. 2004, Kermani et al. 2007; Kim et al. 2009). It is also clear that friction contribute to the wear down process of mechanical systems including robotic joints (Blau 2009). Therefore, friction modeling also becomes a topic of interest for robot condition monitoring and fault detection, see, e.g., (Ray and Remine 1998), (Vemuri and Polycarpou 2004), (McIntyre et al. 2005), (Brambilla et al. 2008), and (Namvar and Aghili 2009).

A Robot joint contains interacting components such as gears, bearings, and shafts, all rotating at different velocities and under different lubrication levels, and as such it is difficult to separate and model friction at component level. A typical approach is to consider the aggregate effects, as a "lumped" joint friction. Friction always opposes motion and in the process converts kinetic energy into heat. Another outcome of friction is wear. Wear is defined as the progressive loss of material from the operating surface of a body occurring as a result of relative motion at its surface (Lansdown et al. 1987).

Friction presents a major challenge for precise control of robotic joints as well as model-based fault detection designs. A precise friction model can improve both control accuracy and fault detection capabilities of MRRs. In this section, the friction model used in both the multiple working mode control of the MRR and the proposed fault detection scheme is described in details.

\subsubsection{Basics of Tribology}

The most important friction characteristics for control and fault detection applications are usually described by the so-called friction curve, which is a plot of friction levels as function of joint velocity. In fact, the friction curve is usually plotted as a function of joint velocity normalized by the ratio of load torques and lubricant viscosity. For simplicity however, it is mostly expressed only as a function of joint velocity. An example of such a plot obtained from experiments on a robot joint is depicted in Fig. 2.4. The nonlinear behavior from low to high speeds for robotic joints is typical in lubricated friction and is known as the Stribeck effect. This phenomenon have been first observed by Stribeck in 1902. The presence of lubricant in the gearboxes (reduction mechanism, i.e, harmonic drive), bearings, and motor shaft causes

\footnotetext{
${ }^{3}$ Lubricated friction is a case of fluid friction where a fluid separates two solid surfaces (Hibbeler 2007).

${ }^{4}$ Skin friction is a component of drag, the force resisting the motion of a solid body through a fluid (Magill 1999).
} 


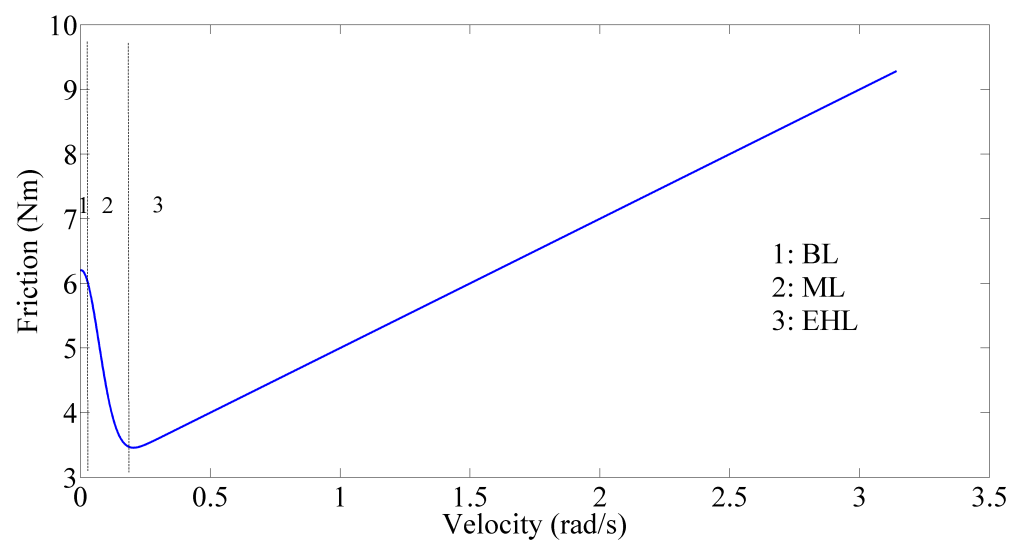

Figure 2.4: Friction curve and the lubrication regimes.

such a behavior in robot joints. Notice that the friction in the motor is dry. Lubricant acts as a separation layer between moving surfaces and therefore could acts as a retarding agent to wear process. By changing the chemical composition of lubricants through additives, the separation layer can even be turned into a chemical barrier between the contact surfaces under high contact pressure, thereby reducing low speed friction and wear. Different types of lubrication regimes segregate the friction curves into three regions, namely the boundary lubrication (BL) 5 mixed lubrication (ML) ${ }^{6}$ and elasto-hydrodynamic lubrication (EHL) 7 . The phenomenon present at very low speeds (BL regime) is caused by the roughness of the surfaces in contact. The lubricant layer between the surfaces increases with velocity while the contact friction decreases until it reaches the full lubrication profile. In EHL regime, the surfaces are completely separated by the lubricant. Friction in EHL region is governed by the lubricant properties like viscosity because it is proportional to the force needed to shear the lubrication layer. The wear processes depend upon contact friction: the more significant the contact friction is, the more significant the wear processes are, i.e. in BL and ML regions. Although the full-film lubrication is theoretically wear free but not perpetually, and wear process resumes after eventual breakdown of this layer. Even the EHL region of the joint friction curve is prone to wear at component level because of

\footnotetext{
${ }^{5}$ Boundary lubrication: The bodies come into closer contact at their asperities; the heat developed by the local pressures causes a condition which is called stick-slip and some asperities break off. Boundary lubrication is also defined as that regime in which the load is carried by the surface asperities rather than by the lubricant.

${ }^{6}$ Mixed lubrication occurs between boundary and elasto-hydrodynamic lubrication, as the name would suggest. The fluid film thickness is slightly greater than the surface roughness, so that there is very little asperity (high point) contact, but the surfaces are still close enough together to affect each other.

${ }^{7}$ Elasto-hydrodynamic lubrication: The opposing surfaces are separated, but there occurs some interaction between the raised solid features called asperities, and there is an elastic deformation on the contacting surface enlarging the load-bearing area whereby the viscous resistance of the lubricant becomes capable of supporting the load.
} 
the differences in their speed at opposing ends, i.e. the input and the output. Differences in speed occur due to the high gear ratio of the gearboxes or harmonic drive transmissions used in robots. This wear process and changes of lubrication properties are the main reasons behind the change of friction parameters.

\subsubsection{Friction Modeling}

The use of empirical-observations based friction models is a norm because of the complexity associated with modeling friction of robot joints. The empirical friction models have had a long history of development and a detailed historical perspective regarding it is provided in (Dowson 1998). The surfaces' asperities are analogous to bristles of a brush at contact level. Each of these (stiff) bristles can be considered as a body in its entirety, having its own individual dynamics similar to that of others in the bulk. Many teams have attempted modeling this dynamic behaviour of friction, some examples of which are in (Hensen et al. 2002), (Harnoy et al. 2008), (Freidovich et al. 2010), and (Zhong et al. 2011). A typical approach is to consider all the dynamics into a single state as in (Dupont et al. 2002). One dynamic friction model commonly used in robotics is the LuGre model, which for a revolute joint is given as:

$$
\begin{aligned}
F r_{i} & =P_{0} z+P_{1} \dot{z}+h\left(\dot{q}_{i}\right) \\
\dot{z} & =\dot{q}_{i}-P_{0} \frac{\left|\dot{q}_{i}\right|}{g\left(\dot{q}_{i}\right)} z
\end{aligned}
$$

where the state $z$ describes the average dynamic behavior of the asperities. It can be interpreted as their average deflection, with stiffness $P_{0}$ and damping $P_{1}$. The dynamic friction model requires the knowledge of $z$ which is not a deterministic quantity. Therefore, the parameters $\left[P_{0}, P_{1}\right]$ describing the dynamical behaviour of friction are difficult to estimate. In practice, considering only a static description of (2.16) is sufficient, which for constant velocities is equivalent to:

$$
F r_{i}\left(\dot{q}_{i}\right)=g\left(\dot{q}_{i}\right) \operatorname{sign}\left(\dot{q}_{i}\right)+h\left(\dot{q}_{i}\right)
$$

The static model is completely described by the $g($.$) and h($.$) functions. In fact, 2.16 is a$ simple extension of 2.17) with the incorporation of dynamics being the only difference.

The function $h\left(\dot{q}_{i}\right)$ describes the friction in EHL region, where friction has a velocity strengthening behavior. In the case of Newtonian fluids, friction is directly proportional to the joint velocity. The viscous behavior of friction is then described by the relation:

$$
h\left(\dot{q}_{i}\right)=B_{i} \dot{q}_{i}
$$


where $B_{i}$ denotes the viscous friction coefficient.

The function $g\left(\dot{q}_{i}\right)$ mathematically represents the friction behavior in the BL and ML regions in which friction has a velocity weakening behavior. $g\left(\dot{q}_{i}\right)$ is usually modeled using the Stribeck's observations, i.e., (Bo and Pavelescu 1982; Jacobson 2003; Woydt and Wäsche 2010), as:

$$
g\left(\dot{q}_{i}\right)=F_{c i}+F_{s i} \exp \left(-\left|\frac{\dot{q}_{i}}{\dot{q}_{s}}\right|^{\alpha_{s}}\right)
$$

where $F_{c i}$ denotes the magnitude of the Coulomb friction related parameter at zero payload, $F_{s i}$ denotes the static friction related parameter, and $\alpha_{s}$ is the exponent of the Stribeck nonlinearity. The resulting static friction model is given by the equation:

$$
F r_{i}(\dot{q}) \triangleq B_{i} \dot{q}_{i}+\left(F_{c i}+F_{s i} \exp \left(-\left|\frac{\dot{q}_{i}}{\dot{q}_{s}}\right|^{\alpha_{s}}\right)\right) \operatorname{sign}\left(\dot{q}_{i}\right)
$$

which describes many of the friction characteristics as a function of joint velocity. The $g($.$) and$ $h($.$) functions are not fixed and one has many different options to choose these functions. The$ LuGre model can be also be rewritten as:

$$
F r_{i}(\dot{q}) \triangleq B_{i} \dot{q}_{i}+\left(F_{c i}+F_{s i} \exp \left(-F_{\tau i} \dot{q}_{i}^{2}\right)\right) \operatorname{sign}\left(\dot{q}_{i}\right)
$$

where $\alpha_{s}=2$ and $F_{\tau i}=\frac{1}{\dot{q}_{s}^{2}}$ is a positive parameter related to the Stribeck effect.

The LuGre model and the corresponding static model were simulated using $g($.$) and h($. functions, chosen according to 2.18) and (2.19), and the response is shown in Fig. 2.5. The simulation was performed with $\dot{q}_{i}$ as half a period of a triangular wave with different slopes $A$. When accelerating, the transition from BL to EHL gives less friction torques than in deceleration. The higher $A$, the more pronounced are the dynamic effects. The parameters are chosen for illustration purposes with static parameters $\left[F_{c i}, F_{s i}, B_{i}, F_{\tau i}, \alpha_{s}\right]=[3,5,2,100,2]$ and dynamic parameters $\left[P_{0}, P_{1}\right]=[2600,170]$.

In this thesis, the joint friction, $F r_{i}$, is modeled such that it includes the same frictional forces described by the LuGre model in equation (2.21); however, to reflect the friction dependency on the payload, the friction is assumed to be function of the joint velocity and the payload that is measured by the integrated joint torque sensor (Hamon et al. 2010).

$$
F r_{i}\left(\dot{q}_{i}, \tau_{s i}\right)=B_{i} \dot{q}_{i}+\left(g_{l}\left(\tau_{s i}\right) F_{c i}+F_{s i} e^{-F_{\tau i} \dot{q}_{i}^{2}}\right) \operatorname{sign}\left(\dot{q}_{i}\right)
$$

The function $g_{l}\left(\tau_{s i}\right)$ is defined as follows,

$$
g_{l}\left(\tau_{s i}\right) \triangleq 1+g_{1}\left|\tau_{s i}\right|+g_{2}\left|\tau_{s i}\right|^{2}
$$




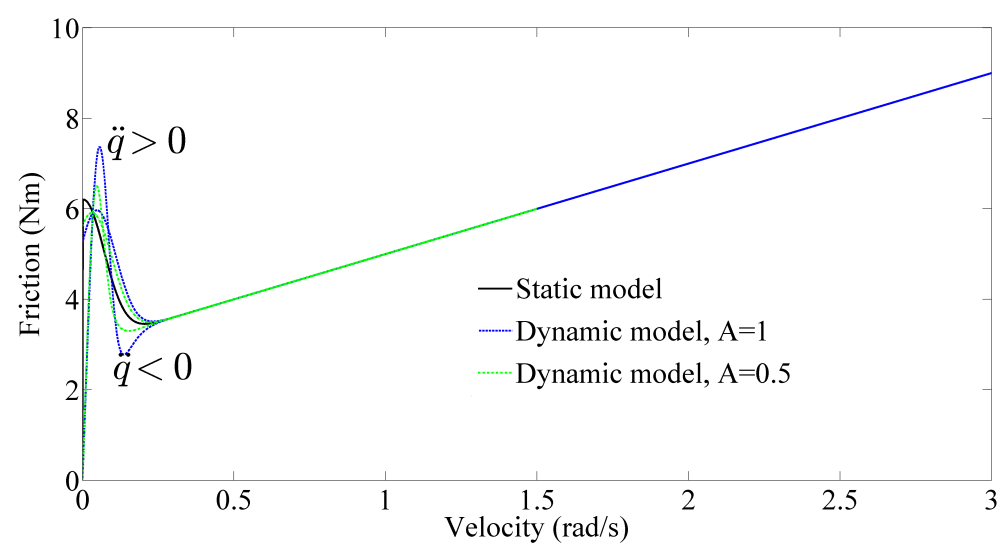

Figure 2.5: LuGre model simulation with different acceleration.

where $g_{1}$ and $g_{2}$ are positive numbers used to emulate the payload-dependent Coulomb friction effect. The payload dependent friction is modeled in quadratic form to better preserve the problem nature (Zhu et al. 2007).

In order to implement the active mode of the multiple working mode control and the proposed fault detection scheme that adaptively updates the joint friction parameters, it is first necessary to linearize the nonlinear friction model around the nominal values of the friction parameters similarly as in (Liu et al. 2004)

$$
F_{s i} e^{-F_{\tau i} \dot{q}_{i}^{2}} \cong \hat{F}_{s i} e^{-\hat{F}_{\tau i} \dot{q}_{i}^{2}}-\tilde{F}_{s i} e^{-\hat{F}_{\tau i} \dot{q}_{i}^{2}}+\hat{F}_{s i} e^{-\hat{F}_{\tau i} \dot{q}_{i}^{2}} \dot{q}_{i}^{2} \tilde{F}_{\tau i}
$$

where $\hat{B}_{i}, \hat{F}_{c i}, \hat{F}_{s i}, \hat{F}_{\tau i}$ denote the nominal values of their respective parameters and the parametric model uncertainty is defined as $\tilde{F}_{i}=\hat{F}_{i}-F_{i}$; therefore,

$$
F_{s i} e^{-F_{\tau i} \dot{q}_{i}^{2}} \cong F_{s i} e^{-\hat{F}_{\tau i} \dot{q}_{i}^{2}}-F_{\tau i} \hat{F}_{s i} \dot{q}_{i}^{2} e^{-\hat{F}_{\tau i} \dot{q}_{i}^{2}}+\hat{F}_{s i} \hat{F}_{\tau i} \dot{q}_{i}^{2} e^{-\hat{F}_{\tau i} \dot{q}_{i}^{2}}
$$

Substituting the "linearized" term $F_{s i} e^{-F_{\tau i} \dot{q}_{i}^{2}}$ of 2.25 into the friction model 2.22 yields:

$$
\begin{aligned}
F r_{i}\left(\dot{q}_{i}, \tau_{s i}\right) & =B_{i} \dot{q}_{i}+\left(g_{l}\left(\tau_{s i}\right) F_{c i}+F_{s i} e^{-\hat{F}_{\tau i} \dot{q}_{i}^{2}} \dot{q}_{i}^{2} e^{-\hat{F}_{\tau i}-F_{\tau i} \hat{F}_{s i} \dot{q}_{i}^{2}}\right) \operatorname{sign}\left(\dot{q}_{i}\right) \\
& +\Phi_{i} \operatorname{sign}\left(\dot{q}_{i}\right)
\end{aligned}
$$

where $\Phi_{i}=\hat{F}_{s i} \hat{F}_{\tau i} \dot{q}_{i}^{2} e^{-\hat{F}_{\tau i} \dot{q}_{i}^{2}}$.

The friction model can be written in a compact form as:

$$
F r_{i}\left(\dot{q}_{i}, \tau_{s i}\right)=Y_{i}\left(\dot{q}_{i}, \tau_{s i}\right) \theta_{i}+\Phi_{i} \operatorname{sign}\left(\dot{q}_{i}\right)
$$


where $Y_{i}\left(\dot{q}_{i}, \tau_{s i}\right) \in \Re^{1 \times 4}$ denotes a known vector whose elements are functions of $\dot{q}_{i}(t)$ and $\tau_{s i}(t) ; \theta_{i} \in \Re^{4}$ contains the uncertain friction parameters.

$$
\begin{gathered}
\theta_{i}=\left[\begin{array}{llll}
B_{i} & F_{c i} & F_{s i} & F_{\tau i}
\end{array}\right]^{T} \\
Y_{i}\left(\dot{q}_{i}, \tau_{s i}\right)=\left[\begin{array}{llll}
\dot{q}_{i} & g_{l}\left(\tau_{s i}\right) \operatorname{sign}\left(\dot{q}_{i}\right) & e^{-\hat{F}_{\tau i} \dot{q}_{i}^{2}} \operatorname{sign}\left(\dot{q}_{i}\right) & -\hat{F}_{s i} \dot{q}_{i}^{2} e^{-\hat{F}_{\tau i} \dot{q}_{i}^{2}} \operatorname{sign}\left(\dot{q}_{i}\right)
\end{array}\right]
\end{gathered}
$$

Remark 3 The joint friction model parameters $\theta_{i}$, defined in (2.27), are not accurately known. However, their nominal values $\hat{\theta}_{i}$ are determined off-line as constants and are assumed to be close to their actual values.

\subsection{Concluding Remarks}

This chapter presented an overview of the diagnosis process. The preliminary concepts of system diagnosis, with special emphasis on the principles of the model-based FDI, are presented. The documented contents focused on describing different concepts related to FDI, with special attention given to methods that are relevant to the proposed fault detection scheme for MRRs. The chapter also introduced friction from both empirical and phenomenological perspectives. Friction depends upon the load and load changes are inevitable not just due to the payloads carried by the robot end-effector but also because of the links of the robot itself. The friction models presented in this chapter take into consideration the dependency of joint friction on both the velocity and the load. These models are crucial not just for designing control laws for MRR but also for detecting its faults. 



\section{Chapter 3}

\section{Multiple Working Mode Control Design}

\subsection{Introduction}

A mobile robot manipulator offering a single working mode may not be appropriate to operate in unstructured environments, and therefore having multiple working modes become a valuable feature. For example, for opening a general door, active mode is necessary for the manipulator to approach the door knob. After the gripper gets hold of the knob, some form of passivity is desirable for the manipulator to prevent the occurrence of large internal forces. The working modes of the MRR joint modules are categorized in two types: passive mode and active mode. The passive mode refers to the mode in which a joint rotates freely with friction compensation. The active mode refers to the mode in which a joint module is working under a selected motion or force control. The decision regarding the selection of active mode is based on task requirements.

\subsection{Dynamic Model of Modular and Reconfigurable Robots with Joint Torque Sensing}

For the research work documented in this thesis, modular and reconfigurable robots comprising of $n$ modules are considered. Each module is integrated with a rotary joint along with a speed reducer and a torque sensor as illustrated in Fig. 3.1.

The following notations are used in the dynamic model:

$I_{m i}:$ rotor moment of inertia about the axis of rotation;

$\gamma_{i}$ : reduction ratio of the speed reducer; 


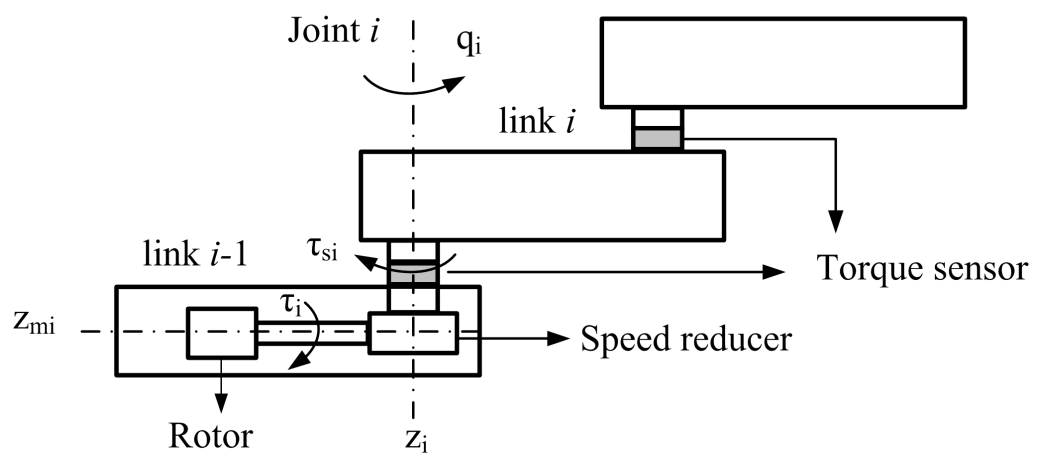

Figure 3.1: Schematic diagram of a joint module.

$q_{i} \quad$ : joint angle;

$\tau_{s i}:$ coupling torque at the torque sensor location;

$\tau_{i} \quad$ : output torque of the rotor;

$z_{m i}$ : unit vector along the axis of rotation of the $i$ th rotor;

$z_{i} \quad:$ unit vector along the axis of rotation of the $i$ th joint; and

$F r_{i}\left(\dot{q}_{i}, \tau_{s i}\right):$ joint friction which is assumed to be function of the joint velocity and the joint load.

The dynamic model of each joint of the modular and reconfigurable robot that was previously formulated in (Liu et al. 2008b) is given by:

For the base module, $i=1$,

$$
I_{m 1} \gamma_{1} \ddot{q}_{1}+F r_{1}\left(\dot{q}_{1}, \tau_{s 1}\right)+\gamma_{1}^{-1} \tau_{s 1}=\tau_{1}
$$

For the second module from the base, $i=2$,

$$
I_{m 2} \gamma_{2} \ddot{q}_{2}+F r_{2}\left(\dot{q}_{2}, \tau_{s 2}\right)+I_{m 2} z_{m 2}^{T} z_{1} \ddot{q}_{1}+\gamma_{2}^{-1} \tau_{s 2}=\tau_{2}
$$

For $i \geq 3$,

$$
\begin{aligned}
& I_{m i} \gamma_{i} \ddot{q}_{i}+I_{m i} \sum_{j=1}^{i-1} z_{m i}^{T} z_{j} \ddot{q}_{j}+F r_{i}\left(\dot{q}_{i}, \tau_{s i}\right)+I_{m i} \sum_{j=2}^{i-1} \sum_{k=1}^{j-1} z_{m i}^{T}\left(z_{k} \times z_{j}\right) \dot{q}_{k} \dot{q}_{j} \\
& +\gamma_{i}^{-1} \tau_{s i}=\tau_{i}
\end{aligned}
$$


The dynamical equation of the $i$ th joint module can be rewritten as:

$$
M_{i} \ddot{q}_{i}(t)+\delta_{i}(t)+Y_{i}\left(\dot{q}_{i}(t), \tau_{s i}(t)\right) \theta_{i}+\Phi_{i}\left(\dot{q}_{i}(t)\right) \operatorname{sign}\left(\dot{q}_{i}(t)\right)+\gamma_{i}^{-1} \tau_{s i}(t)=\tau_{i}(t)
$$

where, $Y_{i}\left(\dot{q}_{i}(t), \tau_{s i}(t)\right), \theta_{i}, \Phi_{i}\left(\dot{q}_{i}(t)\right)$ have been defined in 2.27, and $M_{i}=I_{m i} \gamma_{i}$. The term $\delta_{i}(t) \in \Re$ comprises of coupling effects between lower $i-1$ joints and the $i$ th joint, given by:

$$
\delta_{i}(t)= \begin{cases}0 & \text { for } i=1 \\ I_{m 2} z_{m 2}^{T} z_{1} \ddot{q}_{1}(t) & \text { for } i=2 \\ I_{m i} \sum_{j=2}^{i-1} \sum_{k=1}^{j-1} z_{m i}^{T}\left(z_{k} \times z_{j}\right) \dot{q}_{k}(t) \dot{q}_{j}(t)+I_{m i} \sum_{j=1}^{i-1} z_{m i}^{T} z_{j} \ddot{q}_{j}(t) & \text { for } i \geq 3\end{cases}
$$

Note that the coupling term $\delta_{i}(t)$ comprises of only the $i$ th joint motor dynamics as the links dynamics are captured by the integrated joint torque sensor.

\subsection{Passive Working Mode}

The key to implement passive working mode is the compensation of the joint friction. If friction is compensated, the output shaft of the joint can be moved freely. Based on the motion trend and the angular velocity of the passive joint, a feedforward torque is applied to compensate the joint friction. This proposed passive mode does not require a mechanical clutch to separate the output shaft from the actuator. Since, the actuation chain is never disconnected; the joint can be switched back to active working mode any time at any position without the need to recover from a disconnection.

For the proposed implementation of passive working mode, the requirement for friction compensation is fundamentally different from that for precise tracking control. In order for the joint to work in a passive mode, the joint friction only needs to be compensated such that a small external torque can rotate the joint. In other words, the uncompensated friction should be much smaller compared to the magnitude of the external torque. As shown in Fig. 3.2, friction can be separated into two parts: a constant part and a variable part, and the magnitude of the constant friction part often dominate the overall magnitude of the total friction at low speeds. The constant part of friction is less than the static friction $f_{s i}$ and has the same sign as $f_{s i}$. The sign is determined by the trend of the relative movement or from the torque sensor measurement. With the assumption that the friction dependency on payload is negligible in 


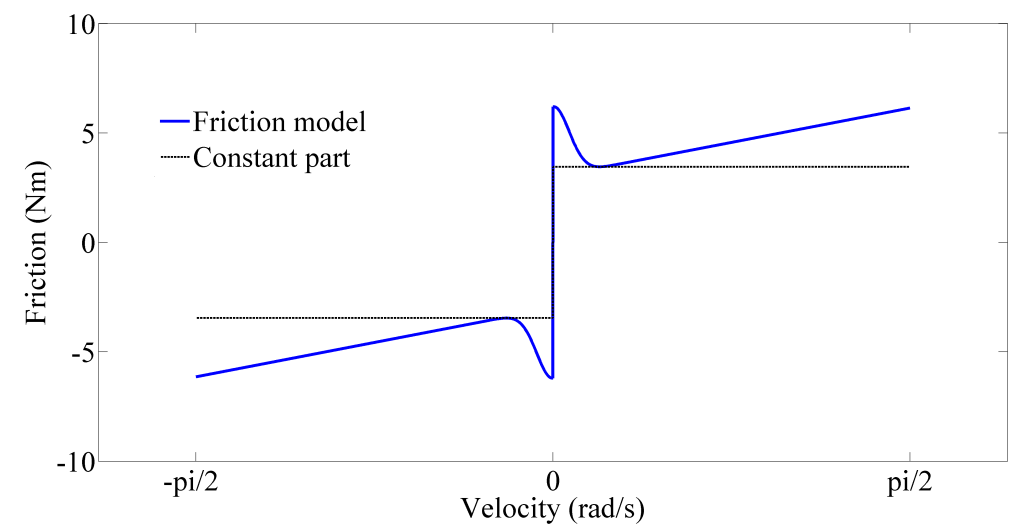

Figure 3.2: Plots of the friction model shows the constant part of the friction.

passive working mode, the friction model given in 2.21 can be used.

$$
F r_{i} \triangleq B_{i} \dot{q}_{i}+\left(F_{c i}+F_{s i} \exp \left(-F_{\tau i} \dot{q}_{i}^{2}\right)\right) \operatorname{sign}\left(\dot{q}_{i}\right)
$$

It should be noted that the motion trend of each joint is usually known or predicable in practical applications and the joint angle and angular speeds are normally measurable. The motion direction of each passive joint is also achievable with the torque sensor measurement. On the basis of motion trend and angular velocity of the passive joint, a feedforward torque can be applied to compensate the friction and enable joint operation in passive mode as follows:

$$
\tau_{i}=F_{m i} \operatorname{sign}\left(\dot{q}_{i}\right), \quad i=1, \ldots n
$$

where $F_{m i}$ denotes the constant part of the friction, which is less than the static friction $F_{s i}$ and its magnitude normally dominates the overall magnitude of the total friction at low speed. The friction can be substantially compensated by using the feedforward torque shown in (3.7).

\section{Experimental Validation}

The proposed passive working mode control has been tested experimentally using the base joint of MRR. The currents for compensation are: $-1.5 A$ for the positive direction and $+1.2 A$ for the negative direction. The external torques required to rotate the MRR joint in the passive mode in two different directions, with and without friction compensation, were measured and given in Table 3.1. As can be observed from Table 3.1, the simple friction compensation can significantly reduce the required external torque needed to rotate the joint by $79 \%$ in the positive direction and $84 \%$ in the negative direction. Such results are adequate for many applications. Friction 
Table 3.1: External torque for MRR joint in passive mode.

\begin{tabular}{|c|c|c|}
\hline Rotate Direction & Positive(0 360 deg) & Negative $\left(\begin{array}{ll}0 & -360 \mathrm{deg}\end{array}\right)$ \\
\hline Torque without compensation (a) & $36.3 \mathrm{Nm}$ & $36.2 \mathrm{Nm}$ \\
\hline Torque with compensation (b) & $7.6 \mathrm{Nm}$ & $5.7 \mathrm{Nm}$ \\
\hline Ratio (b/a) & $21 \%$ & $16 \%$ \\
\hline
\end{tabular}

compensation using a more complete friction model or the torque sensor feedback can further reduce the friction if necessary.

\subsection{Active Working Mode}

With the growing interest in modular and reconfigurable robots, more and more efforts are being made in analysis, modeling, and control of MRRs (Wang et al. 2009), (Liu et al. 2011). For the MRR used to carry out this research, a distributed control method, based on joint torque sensing, was proposed in (Liu et al. 2008a) to control joint modules working under active mode. The control algorithm is comprehended below as a reference.

The joint friction $F r_{i}$ given in 2.26 can be rewritten in the following form:

$$
F r_{i}\left(q_{i}, \dot{q}_{i}\right)=\hat{B}_{i} \dot{q}_{i}+\left(g_{l}\left(\tau_{s i}\right) \hat{F}_{c i}+\hat{F}_{s i} \exp \left(-\hat{F}_{\tau i} \dot{q}_{i}^{2}\right)\right) \operatorname{sign}\left(\dot{q}_{i}\right)+F_{q i}\left(q_{i}, \dot{q}_{i}\right)-Y_{i}\left(\dot{q}_{i}, \tau_{s i}\right) \tilde{F}_{i}
$$

where $B_{i}, F_{c i}, F_{s i}$, and $F_{\tau i}$ have the same implications as those in (3.6), $Y_{i}\left(\dot{q}_{i}, \tau_{s i}\right)$ is defined in (2.27), and $F_{q i}\left(q_{i}, \dot{q}_{i}\right)$ represents the position dependency of friction and other friction modeling errors. $\tilde{F}_{i}$ is defined as:

$$
\tilde{F}_{i}=\left[\begin{array}{llll}
\hat{B}_{i}-B_{i} & \hat{F}_{c i}-F_{c i} & \hat{F}_{s i}-F_{s i} & \hat{F}_{\tau i}-F_{\tau i}
\end{array}\right]^{T}
$$

The nonparametric friction term $F_{q i}\left(q_{i}, \dot{q}_{i}\right)$ is bounded as:

$$
F_{q i}\left(q_{i}, \dot{q}_{i}\right)<\rho_{f q}^{i}
$$

where $\rho_{f q}^{i}$ is a known bound. 


\subsubsection{Control Design}

Let the overall control for each joint be defined by:

$$
\tau_{i}=\frac{\tau_{s i}}{\gamma_{i}}+u_{i}, \quad i=1,2, \ldots, n
$$

where, $u_{i}$ is the $i$ th joint's new control input to be designed.

For the first joint, combining (3.11) with (3.1) yields:

$$
I_{m 1} \gamma_{1} \ddot{q}_{1}+F r_{1}\left(q_{1}, \dot{q}_{1}\right)=u_{1}
$$

For the second joint, combining (3.11) with (3.2) yields:

$$
I_{m 2} \gamma_{2} \ddot{q}_{2}+F r_{2}\left(q_{2}, \dot{q}_{2}\right)+I_{m 2} z_{m 2}^{T} z_{1} \ddot{q}_{1}=u_{2}
$$

For $i \geq 3$, substituting (3.11) with (3.3) yields:

$$
I_{m i} \gamma_{i} \ddot{q}_{i}+F r_{i}\left(q_{i}, \dot{q}_{i}\right)+I_{m i} \sum_{j=1}^{i-1} z_{m i}^{T} z_{j} \ddot{q}_{j}+\sum_{j=2}^{i-1} \sum_{k=1}^{j-1} z_{m i}^{T}\left(z_{k} \times z_{j}\right) \dot{q}_{k} \dot{q}_{j}=u_{i}
$$

First, the following system errors are defined,

$$
\begin{gathered}
e_{i} \triangleq q_{i}-q_{i d} \\
r_{i} \triangleq \dot{e}_{i}+\lambda_{i} e_{i} \\
a_{i} \triangleq \ddot{q}_{i d}-2 \lambda_{i} \dot{e}_{i}-\lambda_{i}^{2} e_{i}
\end{gathered}
$$

where $\lambda_{i}$ is a positive constant. The following two properties are worth noting for control law design.

Property 1 Since $z_{m i}$ and $z_{i}$ are unit vectors along the direction of rotation of the ith rotor and joint, the resulting vector products are bounded as:

$$
\left|z_{m i}^{T} z_{j}\right| \leq 1 \quad\left|z_{m i}^{T}\left(z_{k} \times z_{j}\right)\right| \leq 1
$$

Property 2 The velocity and acceleration of a stabilized joint is bounded, i.e., if the ith joint 
is stabilized, then it implies that:

$$
\left|\dot{q}_{i}\right| \leq \rho_{v i} \quad\left|\ddot{q}_{i}\right| \leq \rho_{a i}
$$

where $\rho_{v i}$ and $\rho_{a i}$ are known constant bounds.

Similar to (Liu et al. 2008a), the parametric uncertainty $\tilde{F}_{i}$ can be decomposed as:

$$
\tilde{F}_{i}=\tilde{F}_{c}^{i}+\tilde{F}_{v}^{i}
$$

where $\tilde{F}_{c}^{i}$ is a constant unknown vector, and $\tilde{F}_{v}^{i}$ is the variable parametric model uncertainty having the upper bound as defined in 3.19 .

$$
\left|\tilde{F}_{v}^{i}\right|<\rho_{f v}^{i}
$$

It is well known that an adaptive compensator can be designed to compensate for the constant parametric uncertainty $\tilde{F}_{c}^{i}$ and a robust compensator can be designed for the variable part $\tilde{F}_{v}^{i}$. By applying the decomposition-based control design approach developed in (Liu et al. 1996), (Liu 2002), the following control input is designed for stabilizing the first joint.

$$
u_{1}=I_{m 1} \gamma_{1} a_{1}+\hat{B}_{1} \dot{q}_{1}+\left(\hat{F}_{c 1}+\hat{F}_{s 1} \exp \left(\hat{F}_{\tau 1} \dot{q}_{1}^{2}\right)\right) \operatorname{sign}\left(\dot{q}_{1}\right)+u_{u}^{1}+Y_{i}\left(\dot{q}_{1}, \tau_{s 1}\right)\left(u_{p c}^{1}+u_{p v}^{1}\right)
$$

where $u_{u}^{1}, u_{p c}^{1}, u_{p v}^{1}$ are designed to compensate for the nonparametric uncertainty $F_{q i}(q, \dot{q})$, constant parametric uncertainty $\tilde{F}_{c}^{i}$, and variable parametric uncertainty $\tilde{F}_{v}^{i}$, respectively. The compensators $u_{u}^{i}, u_{p v}^{i}$, and $u_{p c}^{i}$ are defined as in (Liu et al. 2008a).

$$
\begin{aligned}
& u_{u}^{i}=\left\{\begin{array}{l}
-\rho_{f q}^{i} \frac{r_{i}}{\left|r_{i}\right|}, \quad\left|r_{i}\right|>\varepsilon_{u}^{i} \\
-\rho_{f q\left|r_{u}^{i}\right|}^{i},\left|r_{i}\right| \leq \varepsilon_{u}^{i}
\end{array}\right. \\
& u_{p c}^{i}=-k_{1} \int_{0}^{t} Y_{i}\left(\dot{q}_{i}, \tau_{s i}\right)^{T} r_{i} d \tau \\
& u_{p v}^{i}=\left\{\begin{array}{l}
-\rho_{f v}^{i} \frac{\varsigma^{i}}{\left|\varsigma^{i}\right|},\left|\varsigma^{i}\right|>\varepsilon_{p}^{i} \\
-\rho_{f v}^{i} \frac{\varsigma^{i}}{\left|\varepsilon_{p}^{i}\right|},\left|\varsigma^{i}\right| \leq \varepsilon_{p}^{i}
\end{array}\right.
\end{aligned}
$$

where $\varsigma^{i}=Y_{i}\left(\dot{q}_{i}, \tau_{s i}\right)^{T} r_{i} \cdot \varepsilon_{u}^{i}, \varepsilon_{p}^{i}$ are positive control parameters. 
The coupling term $I_{m i} \sum_{j=1}^{i-1} z_{m i}^{T} z_{j} \ddot{q}_{j}$ in 3.2 can also be rewritten as:

$$
\begin{aligned}
I_{m i} \sum_{j=1}^{i-1} z_{m i}^{T} z_{j} \ddot{q}_{j} & =\sum_{j=1}^{i-1}\left[\begin{array}{ll}
I_{m i} \hat{\theta}_{j}^{i} & I_{m i}
\end{array}\right]\left[\begin{array}{c}
\ddot{q}_{j} \\
\tilde{\theta}_{j}^{i} \ddot{q}_{j}
\end{array}\right] \\
& \triangleq \sum_{j=1}^{i-1} I_{j}^{i} D_{j}^{i}
\end{aligned}
$$

where $\hat{\theta}_{j}^{i}$ denotes the dot product of the unit vectors $z_{m i}$ and $z_{j}$, and $\tilde{\theta}_{j}^{i}$ is the alignment error given by the difference in dot products of the nominal and actual direction vectors. Considering the variable parametric uncertainty in the coupling term $I_{m i} \sum_{j=1}^{i-1} z_{m i}^{T} z_{j} \ddot{q}_{j}, D_{j}^{i}$ in 3.24 can be decomposed into a constant plus a bounded variable term as:

$$
D_{j}^{i}=D_{j c}^{i}+D_{j v}^{i}
$$

and the variable term is bounded as:

$$
\left|D_{j v}^{i}\right| \leq \rho_{j d}^{i}
$$

Similar to (Liu et al. 2008a), an adaptive compensator $u_{j c}^{i}$ and a robust compensator $u_{j v}^{i}$ are designed respectively for the constant uncertainty term $D_{j c}^{i}$ and for the variable term $D_{j v}^{i}$, as follows:

$$
\begin{gathered}
u_{j c}^{i}=-k_{2} \int_{0}^{t} I_{j}^{i T} r_{i} d \tau \\
u_{j v}^{i}=\left\{\begin{array}{l}
-\rho_{j d}^{i} \frac{\sigma_{j}^{i}}{\left|\sigma_{j}^{i}\right|}, \quad\left|\sigma_{j}^{i}\right|>\varepsilon_{d}^{i} \\
-\rho_{j d}^{i} \frac{\sigma_{j}^{i}}{\left|\varepsilon_{d}^{i}\right|},\left|\sigma_{j}^{i}\right| \leq \varepsilon_{d}^{i}
\end{array}\right.
\end{gathered}
$$

where $\sigma_{j}^{i}=I_{j}^{i T} r_{i}$, and $\varepsilon_{d}^{i}$ is a positive control parameter.

The coupling term $I_{m i} \sum_{j=2}^{i-1} \sum_{k=1}^{j-1} z_{m i}^{T}\left(z_{k} \times z_{j}\right) \dot{q}_{k} \dot{q}_{j}$ present in the dynamic model of the $i$ th joint 3.2 can also be rewritten as:

$$
\begin{aligned}
I_{m i} \sum_{j=2}^{i-1} \sum_{k=1}^{j-1} z_{m i}^{T}\left(z_{k} \times z_{j}\right) \dot{q}_{k} \dot{q}_{j} & =\sum_{j=2}^{i-1} \sum_{k=1}^{j-1}\left[\begin{array}{ll}
I_{m i} \hat{\Theta}_{k j}^{i} & I_{m i}
\end{array}\right]\left[\begin{array}{c}
\dot{q}_{k} \dot{q}_{j} \\
\tilde{\Theta}_{k j}^{i} \dot{q}_{k} \dot{q}_{j}
\end{array}\right] \\
& \triangleq \sum_{j=2}^{i-1} \sum_{k=1}^{j-1} J_{k j}^{i} P_{k j}^{i}
\end{aligned}
$$


where the term $P_{k j}^{i}$ can be decomposed as:

$$
P_{k j}^{i}=P_{k j c}^{i}+P_{k j v}^{i}
$$

and the variable term $P_{k j v}^{i}$ is bounded by:

$$
\left|P_{k j v}^{i}\right| \leq \rho_{k v} \rho_{j v}
$$

By applying decomposition-based control design technique, an adaptive compensator $V_{k j c}^{i}$ is designed for the constant uncertainty term $P_{k j c}^{i}$ and a robust compensator $V_{k j v}^{i}$ is designed for the variable part $P_{k j v}^{i}$ similar to (Liu et al. 2008a). The terms $V_{k j c}^{i}$ and $V_{k j v}^{i}$ are defined as:

$$
\begin{gathered}
V_{k j c}^{i}=-k_{3} \int_{0}^{t} J_{k j}^{i}{ }^{T} r_{i} d \tau \\
V_{k j v}^{i}=\left\{\begin{array}{l}
-\rho_{k v} \rho_{j v} \frac{\beta_{k j}^{i}}{\left|\beta_{k j}^{i}\right|},\left|\beta_{k j}^{i}\right|>\varepsilon_{v}^{i} \\
-\rho_{k v} \rho_{j v} \frac{\beta_{k j}^{i}}{\left|\varepsilon_{p i}^{i}\right|},\left|\beta_{k j}^{i}\right| \leq \varepsilon_{v}^{i}
\end{array}\right.
\end{gathered}
$$

where $\beta_{k j}^{i}=J_{k j}^{i}{ }^{T}$ and $\varepsilon_{v}^{i}$ is a positive control parameter. The control law 3.20 ensures the stability of first joint, which in turn guarantees the boundedness of the tracking errors, and therefore, the boundedness of the magnitudes of $\dot{q}_{1}$ and $\ddot{q}_{1}$. Since $\ddot{q}_{1}$ is bounded, a saturationbased robust compensator can be used to compensate for the effects of $\ddot{q}_{1}$. Therefore, the control input $u_{2}$, given below, for the second joint would be given by the control law derived in (Liu 2002) with an additional term $I_{1}^{2}\left(u_{1 c}^{2}+u_{1 v}^{2}\right)$ to compensate for the effects of $\ddot{q}_{1}$.

$$
\begin{aligned}
u_{2} & =I_{m 2} \gamma_{2} a_{2}+\hat{B}_{2} \dot{q}_{2}+\left(\hat{F}_{c 2}+\hat{F}_{s 2} \exp \left(\hat{F}_{\tau 2} \dot{q}_{2}^{2}\right)\right) \operatorname{sign}\left(\dot{q}_{2}\right) \\
& +u_{u}^{2}+I_{1}^{2}\left(u_{1 c}^{2}+u_{1 v}^{2}\right)+Y_{i}\left(\dot{q}_{2}, \tau_{s 2}\right)\left(u_{p c}^{2}+u_{p v}^{2}\right)
\end{aligned}
$$

Similarly, the control input $u_{i}$ for the $i$ th joint is given by following expression:

$$
\begin{aligned}
u_{i} & =I_{m i} \gamma_{i} a_{i}+\hat{B}_{i} \dot{q}_{i}+\left(\hat{F}_{c i}+\hat{F}_{s i} \exp \left(\hat{F}_{\tau_{i}} \dot{q}_{i}^{2}\right)\right) \operatorname{sign}\left(\dot{q}_{i}\right)+u_{u}^{i}+Y_{i}\left(\dot{q}_{i}, \tau_{s i}\right)\left(u_{p c}^{i}+u_{p v}^{i}\right) \\
& +\sum_{j=1}^{i-1} I_{j}^{i}\left(u_{j c}^{i}+u_{j v}^{i}\right)+\sum_{j=2}^{i-1} \sum_{k=1}^{j-1} J_{k j}^{i}\left(V_{k j c}^{i}+V_{k j v}^{i}\right)
\end{aligned}
$$

The last two terms in (3.35) are the compensators for the velocities and accelerations of the lower joints. It has been proved in (Liu et al. 2008a) that the tracking error is uniformly bounded under the control law defined by (3.11), (3.20), (3.34), and (3.35). 


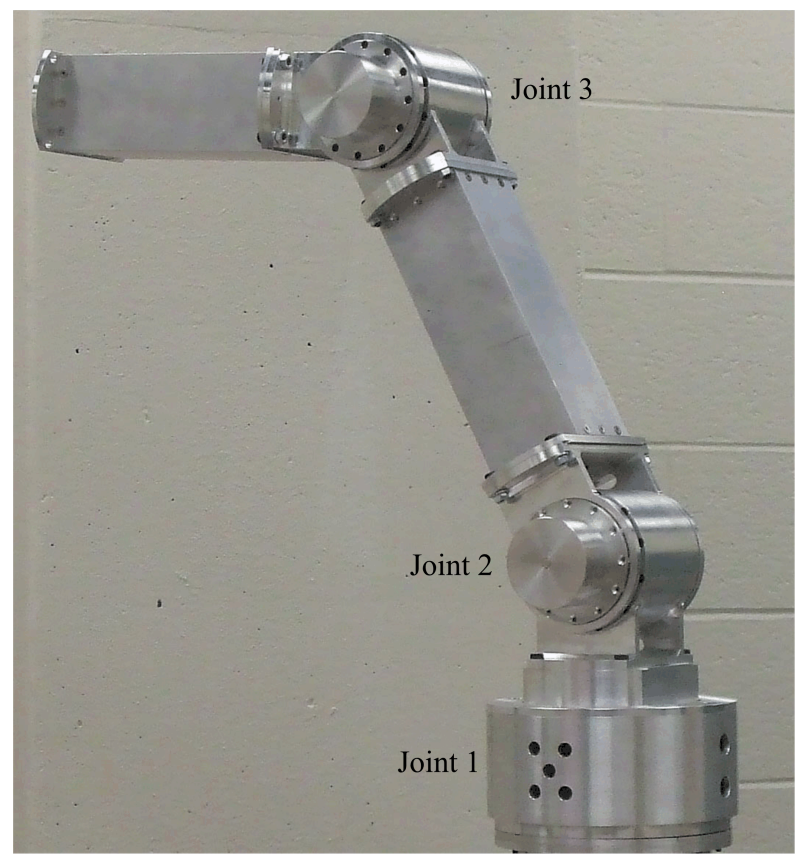

Figure 3.3: 3-DOF modular and reconfigurable robot.

\subsubsection{Active Working Mode Experimental Results}

The active working mode was implemented on a 3-DOF, revolute MRR shown in Fig. 3.3 the dynamics of which are described by Equations (3.1) to (3.3). The dynamic model's parameters, measured in SI units in the joint space, are listed in Table 3.2. While the friction model parameters were determined experimentally, the rotor inertia and harmonic drive's reduction ratio of each joint were obtained from manufacturer's specification sheet. Both, the reference and measured position trajectories of the three joint are depicted in Fig. 3.4. The tracking errors at the three joints are shown in Fig. 3.5. The commanded torque at the three joints are shown in Fig. 3.7 and the torque sensor measurements are shown in Fig. 3.6. 


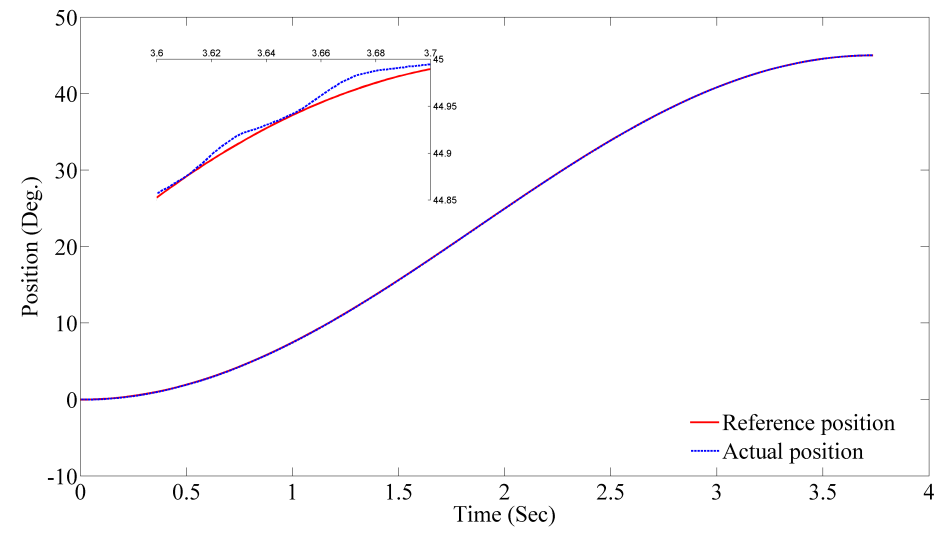

(a) Joint 1

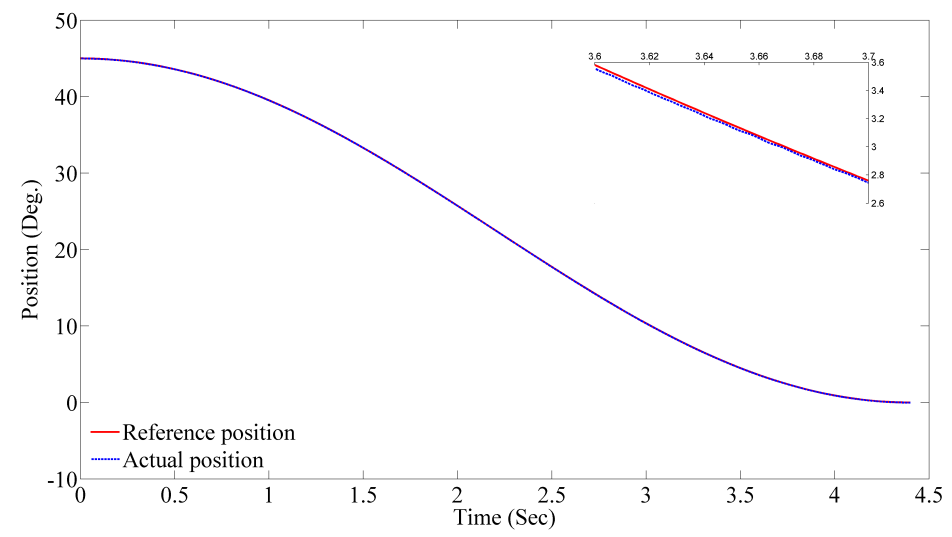

(b) Joint 2

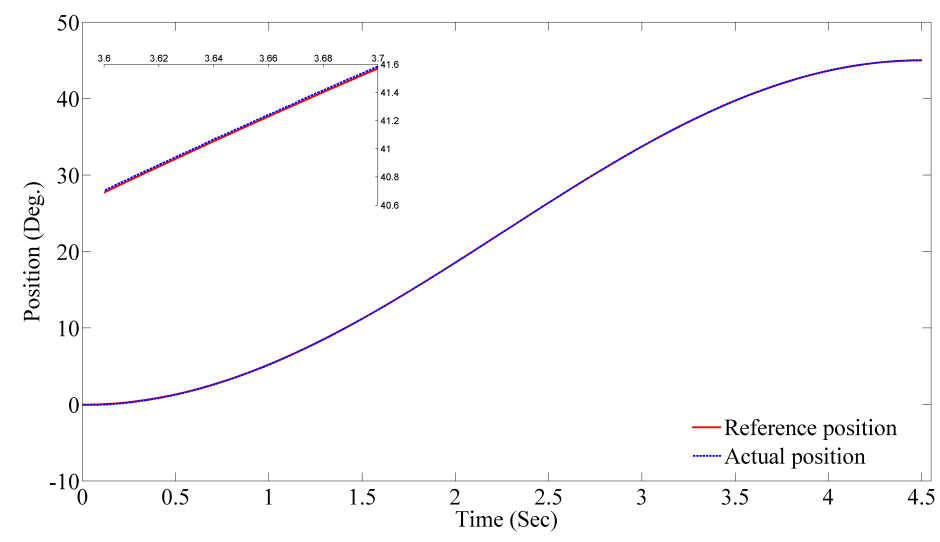

(c) Joint 3

Figure 3.4: Position tracking for the 3-DOF MRR. 


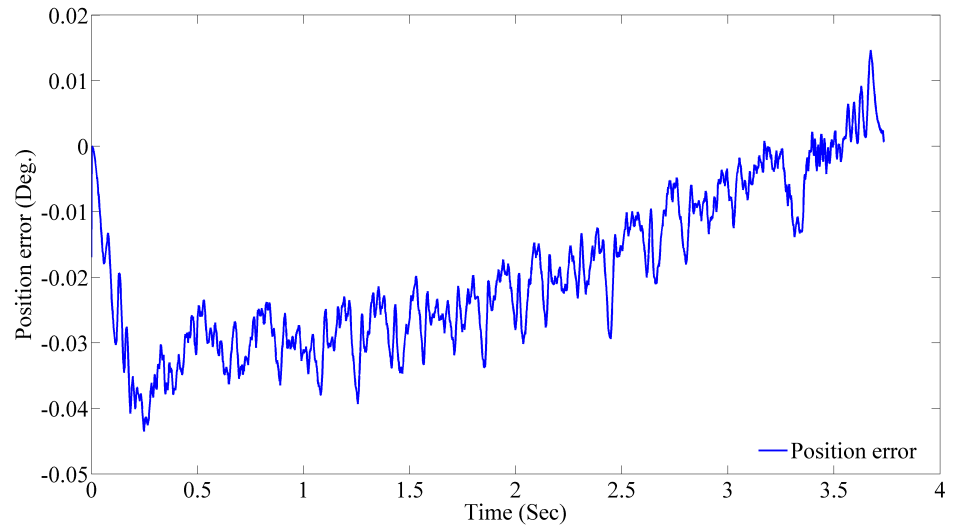

(a) Joint 1

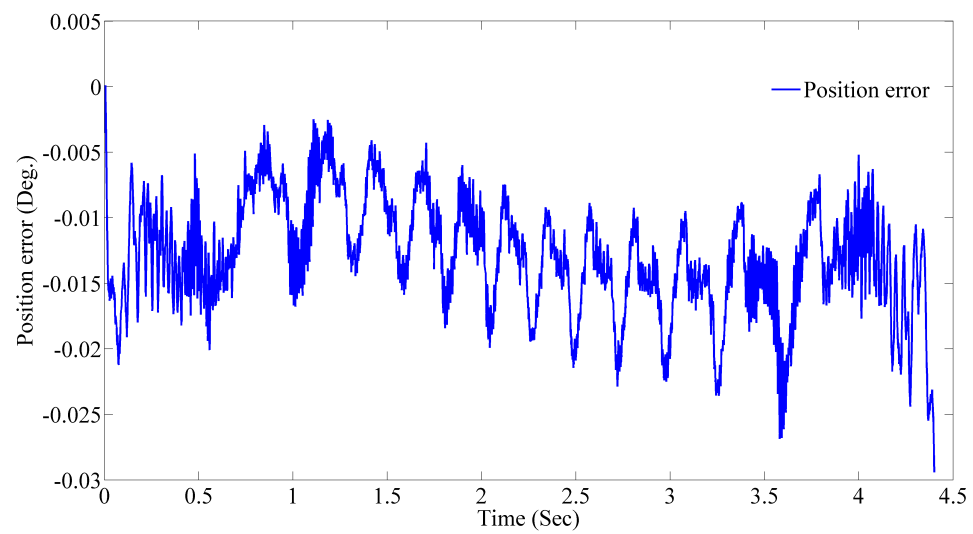

(b) Joint 2

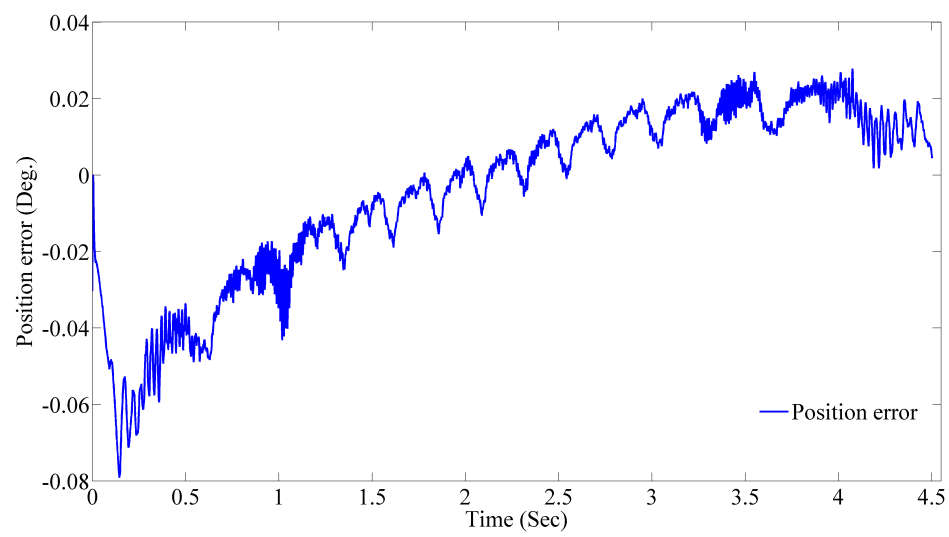

(c) Joint 3

Figure 3.5: Position tracking errors for the 3-DOF MRR. 


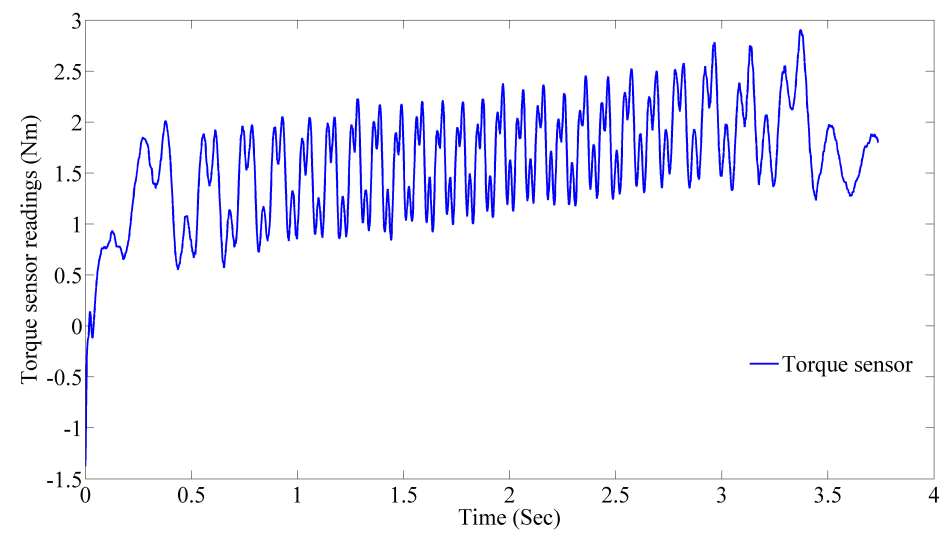

(a) Joint 1

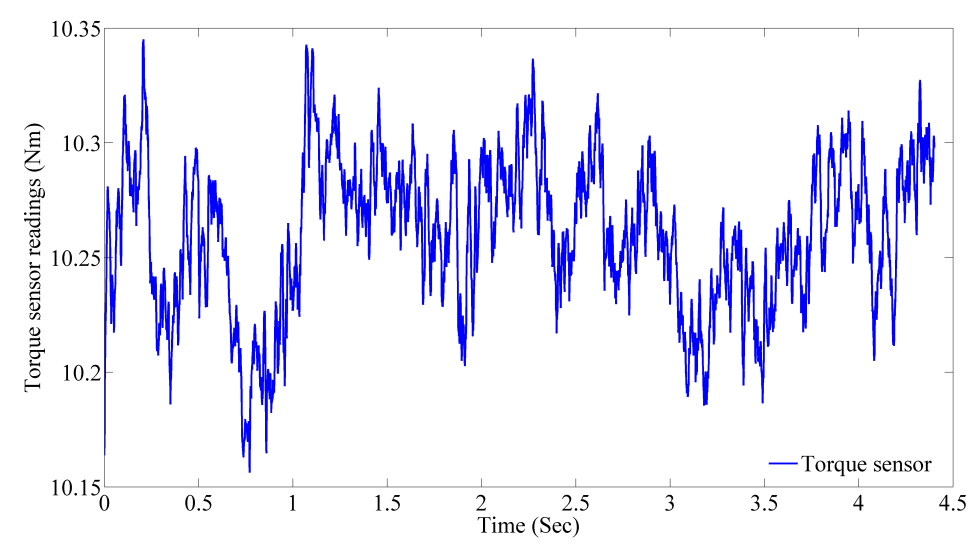

(b) Joint 2

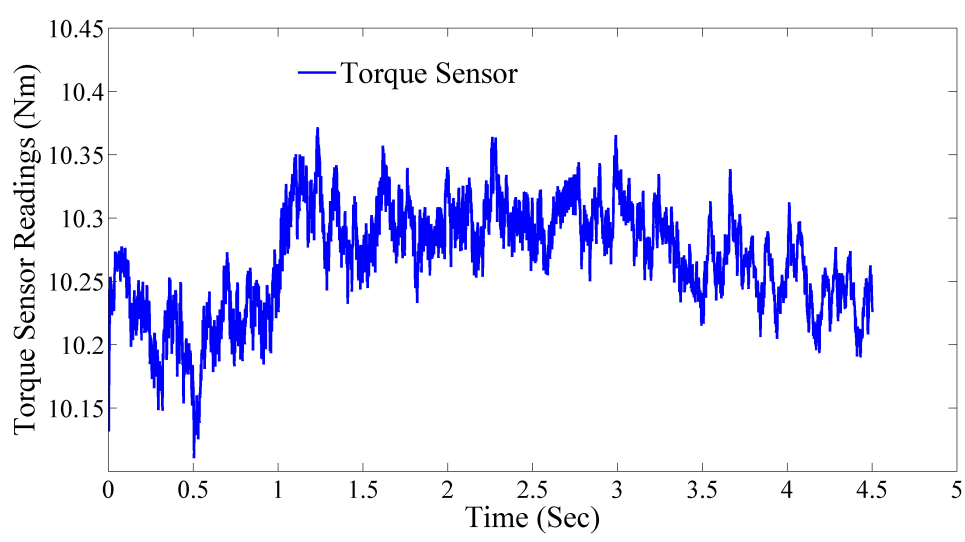

(c) Joint 3

Figure 3.6: Torque sensor readings for the 3-DOF MRR. 


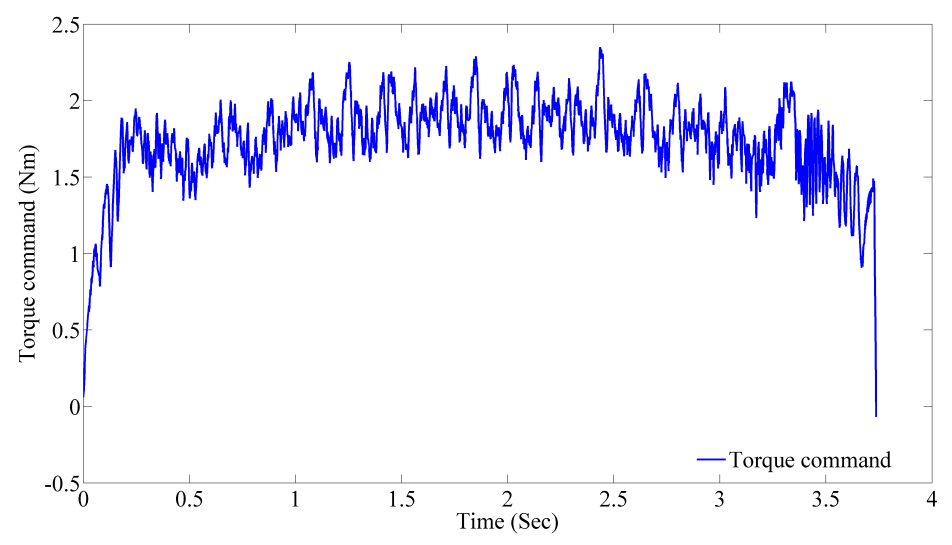

(a) Joint 1

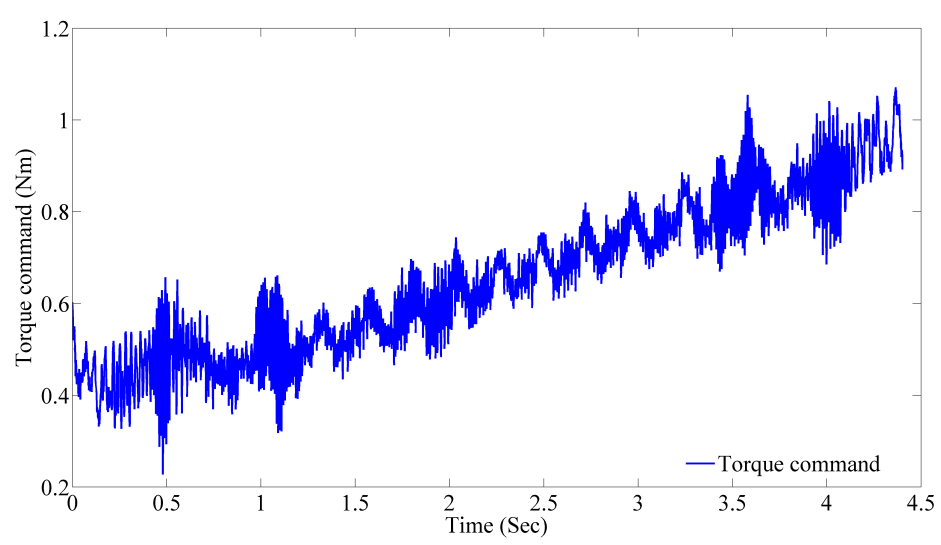

(b) Joint 2

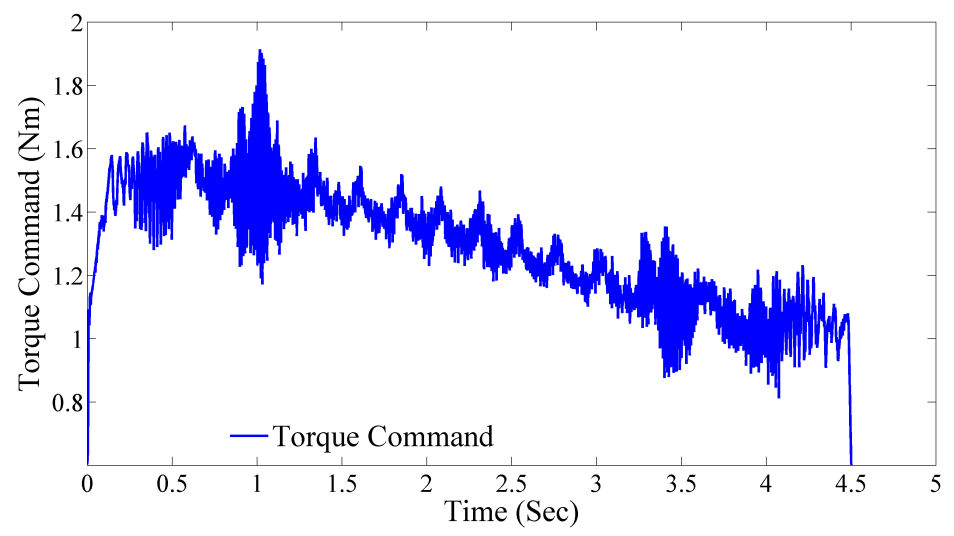

(c) Joint 3

Figure 3.7: Torque commands for the 3-DOF MRR. 
Table 3.2: Physical parameters of the 3-DOF MRR.

\begin{tabular}{|c|c|c|c|}
\hline Parameter & Joint 1 & Joint 2 & Joint 3 \\
\hline \hline \multicolumn{3}{|c|}{ Datasheet-based parameters } \\
\hline Rotor inertia (kg-m2) & $0.168 \times 10^{-4}$ & $0.168 \times 10^{-4}$ & $0.168 \times 10^{-4}$ \\
\hline Gear ratio & 101 & 101 & 101 \\
\hline \multicolumn{2}{|c|}{ Experimentally identified friction parameters } \\
\hline Viscous friction coefficient (Nms/rad) & 0.24 & 0.12 & 0.1 \\
\hline Static friction parameter (Nm) & 0.0324 & 0.0152 & 0.0143 \\
\hline Coulomb friction parameter (Nm) & 0.025 & 0.015 & 0.0124 \\
\hline Stribeck effect parameter (Nm) & 85 & 100 & 90 \\
\hline$g_{1}$ & 5.1 & 4.9 & 5.2 \\
\hline$g_{2}$ & 0.65 & 0.6 & 0.8 \\
\hline
\end{tabular}

\subsection{Concluding Remarks}

This chapter described the multiple working mode control design concepts which form the backbone of the door opening strategy proposed in this thesis. The working modes that were utilized in this research are: passive and active working modes. However, the multiple working mode control is not limited to these two working modes only. Different control strategies can be formulated and implemented to add more working modes as desired; for example, compliance control or teleoperated mode. The passive and active working modes were described in details and experimental result were presented. 



\section{Chapter 4}

\section{Multiple Working Mode Control of Door Opening with a Mobile Modular and Reconfigurable Robot}

\subsection{Introduction}

Modern robot applications, including mobile manipulation, have far higher performance demands than those of assembly and repetitive tasks. Mobile manipulators become more useful when they perform tasks that are of secondary nature to humans, such as door opening. Humans can readily tell a door's opening direction and then apply appropriate force to open the door. In doing so, they instinctively use vision, memory, and/or trial-and-error to quickly estimate the radius of the door and the position of the hinge. The human brain processes the gathered information to help determine the correct direction of the force application. Robot manipulators, however, are not gifted with all the senses that humans have been by nature. Performing the same tasks with mobile robot manipulators is quite challenging and usually requires sophisticated control and/or mechanical designs for adequate performance.

One of the fundamental requirements for successfully opening a door with a mobile manipulator is the proper handling of the interaction between the manipulator and the door. The interaction forces sensed at the robot's end-effector are of concern. Because of the inevitable position errors of the mobile base, large internal forces can be generated. Excessive internal forces are undesirable, as they may cause the task to fail or may even cause damage to the hardware.

Conventional robot manipulators use position control and need specific programming that is tailored to precise requirements of a task, with poor adaptability to changes in the environment 
and great sensitivity to modeling errors. In addition, precise path or trajectory planning for robot manipulators requires accurate kinematics modeling, which is not always possible for a mobile manipulator. Introduction of force-feedback control in robot manipulators gives advantages of versatility and adaptability but has its own problems when implemented in real time. Since force sensors detect external forces by amplifying the strain of strain gauges, signal noise is also amplified. High-frequency noise can be reduced by using low-pass filters; however, the frequency band of force sensing becomes very narrow (Katsura et al. 2007). Filtering the force signal also adds an unacceptable lag. Therefore, stability problems and severe performance degradation may result.

To handle the problem of excessive internal forces, existing literature has focused on the use of compliance control and multi-tactile sensors. Some researchers chose to explore new designs of compliant mechanisms that lead to a robotic system dedicated to the door opening task (Kobayashi et al. 2008), (Lawrence and Erik 2008). For the door opening task, active working mode is required for the manipulator to approach and grasp the door knob. During the door opening process, some form of passivity is desirable to prevent the occurrence of large internal forces.

The focus of this research work is to pull a door knob to open a door. Unlike existing methods, the proposed work presents a new approach to the door opening problem in the sense that it does not require complicated compliance control design or any special mechanical design of the robot end-effector. The proposed approach relies on the multiple working modes of the MRR joints to prevent the occurrence of large internal forces by choosing some joints to work in passive mode. After grasping and rotating the door knob by the MRR, multiple working mode control is used to slightly open the door by only moving the MRR. While in this process, the initial position of the mobile base and the door parameters are estimated using the proposed parameter estimation method. These estimated parameters are then used for planning a safe path of the mobile base. Afterwards, the mobile base starts following the desired path while the MRR is working under multiple working mode control to prevent the occurrence of large internal forces.

\subsection{Related Work}

Research works reported in the literature of door opening control using a mobile robot manipulator, mostly based on a combination of position and force control. Nagatani and Yuta (1994. 1995, 1996) proposed a strategy for opening doors that makes use of analytical description of the door handle trajectory. In their research, with the assumption of the position and radius of the door known, they applied the concept of action primitives to door opening. Each action 
primitive is equipped with an error adjustment mechanism to cope with accumulated position errors of the mobile base. Niemeyer and Slotine (1997) proposed a control method of following the path of least resistance to solve the problem of door opening, which does not require a kinematic model of the door but needs high-resolution joint velocity measurement. Petersson, et al. (2000) proposed a high-level control approach that uses off-the-shelf algorithms for force/torque control for door opening tasks using mobile manipulators. Their results demonstrate that the control design using hybrid dynamic system models can reduce error magnitudes considerably during the door handle grasping task. Kragic, et al. (2002) demonstrated an intelligent control architecture for finding the doorknob through visual servoing and then estimating the door parameters. In their proposed method, they integrated vision and control system where the objective is to provide information required to switch between control modes of different complexity. Waarsing, et al. (2003) proposed a behaviour-based controller for door opening with a mobile manipulator. The key for realizing such a behaviour-based controller is the cooperation among the mobile manipulator sub-systems, that is, the locomotion and the manipulator control system. Chung, et al. (2009) designed a multi-fingered robotic hand for an indoor service robot PSR1. In that work, active sensing algorithms are proposed to reduce the effect of uncertainties in the environment. Fingertip tactile sensors are used for estimating the contact force and the contact position. The computed contact force is used to carry out compliance control of the door opening process. Kim, et al. (2004) developed a special mobile manipulator called Hombot for opening a door. Hombot is equipped with an anthropomorphous arm with a double active universal joint (DAUJ) to guarantee a compact size of the manipulator. Compliant control is used for the relaxation of the end-effector to successfully accomplish the door opening task. Brooks, et al. (2004) developed a mobile robot manipulator system called CARDEA to push open doors. It relies on behavior and sensorimotor control where behaviors of the robot here refer to actions like following a corridor, or approaching a door. CARDEA uses a SEGWAY base mechanism for locomotion equipped with a 15-DOF robotic arm. The robotic arm is designed for an unstructured environment and comprises of a series of elastic actuators to analyze the response of behavior-based methods and dexterous manipulation. Through virtual spring models, human-like response was obtained and the results were experimentally verified. However, this robot is capable of opening ajar doors only, as it is not designed for grasping and turning the door handle or knob. Klingbeil, et al. (2008) developed a vision-based learning algorithm capable of opening various types of doors without prior knowledge of the door parameters. Kobayashi, et al. (2008) have been developing a series of rescue robots named UMRS (Utility Mobile Robots for Search) since the Great Hanshin-Awaji earthquake in 1995. In their recent version, they implemented a door opening system using compliant mechanisms.

To the best of our knowledge, the reported works are all based on mobile manipulators 
equipped with conventional robotic arms working only in active mode. As a result, compliance control approaches involving force/position control algorithms are demanded to reduce the effects of the internal forces at the end-effector resulting from the interaction with the door. A modular and reconfigurable robot has been developed in house at the Systems and Control laboratory that has joint modules supporting multiple working modes, specifically the active and passive working modes (Liu et al. 2008). By carefully choosing when and which joint module should be switched between these two working modes, the problem of door opening is addressed in this thesis without the need for compliance control and with less sensory and computational complexity.

\subsection{Problem Formulation}

The focus of this research work is on the door opening and we assume that the reaching/grasping task has been accomplished. The robot end-effector is constrained to move along the circular trajectory of the door knob throughout the door opening task. When using conventional robots with joints capable of working only in active mode, the occurrence of large internal forces is inevitable due to the position and modeling errors. Compliant control algorithms are therefore a requirement for door opening tasks, but they come at complexity and computational burden. A planar model of the door opening process is depicted in Fig. 4.1. The door knob motion is assumed to follow an arc trajectory in the $x-y$ plane with a center of rotation at $\left(x_{0}, y_{0}\right)$ and a radius $r$. With the assumption that the gripper holds the door knob firmly, the knob's position forms the gripper's trajectory during door opening process. The following relation has to hold at all times:

$$
r^{2}=\left(x-x_{0}\right)^{2}+\left(y-y_{0}\right)^{2}
$$

With the robot supporting only active working mode, the methods for control during the described door opening task may be summarized as follows. Assuming that the absolute path of the door knob is accurately known, one approach is to command the robot to follow the door knob path using a standard position or trajectory controller. This is perhaps the most impractical approach as small position errors of the mobile manipulator may create large internal forces. An alternate approach is to include the door mechanism into the full dynamic model of the manipulator as an over-actuated closed kinematic chain (Li and Slotine 1989), and then use the extra motors to control the internal forces. While theoretically justifiable, this again is intolerant of kinematic modeling errors. To deal with the problem of large internal forces, complicated control techniques such as compliant motion control or predictive control are studied to relax the internal forces (Jain and Kemp 2010). These control techniques are 


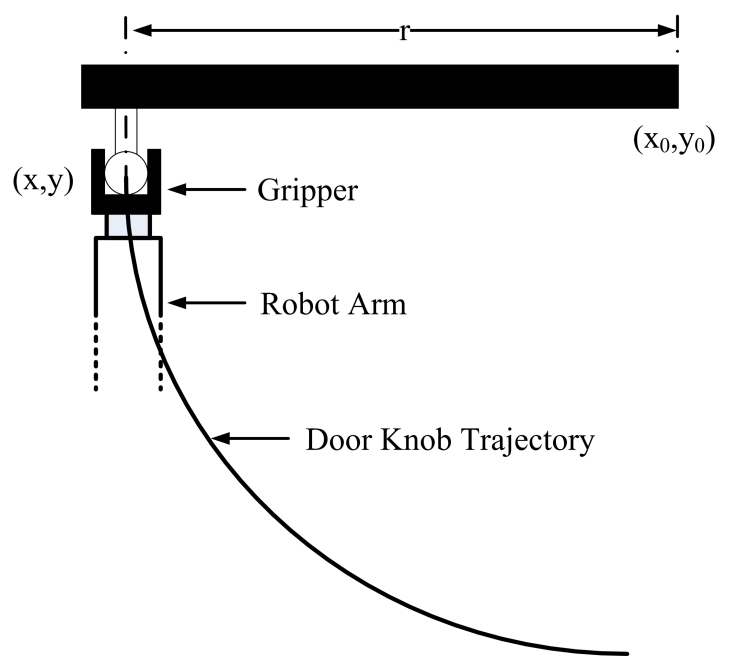

Figure 4.1: A planar model of door opening.

based on force-feedback control and usually have a slow response and a high computational cost.

We propose a new approach to the door opening problem that is based on the framework of multiple working mode control proposed in (Liu et al. 2008), (Ahmad and Liu 2010). The multiple working mode control is used to prevent the occurrence of large internal forces by switching between active and passive working modes.

\subsection{Description of the Mobile MRR}

\subsubsection{Modular and Reconfigurable Robot}

Four MRR modules plus a gripper, providing a total of five degrees of freedom, have been developed locally at Systems and Control laboratory. The MRR has been successfully integrated with a mobile platform to experimentally validate the theoretical developments of this research on mobile MRRs. A picture of the MRR is shown in Fig. 4.2. Each joint module consists of a brushless DC motor, an encoder, a braking system, homing and limit sensors, a harmonic drive with an integrated torque sensor, and an amplifier. A six-axis force sensor is mounted on the MRR wrist module. Multiple working modes are implemented on each joint module; each module can, therefore, independently work in active mode with position or force control, or passive mode with friction compensation. By sending commands to the DSP card embedded, the corresponding joint module can be switched online to work in either the active or the passive mode. The MRR wrist is developed based on the 2-DOF double active universal joint (DAUJ) 


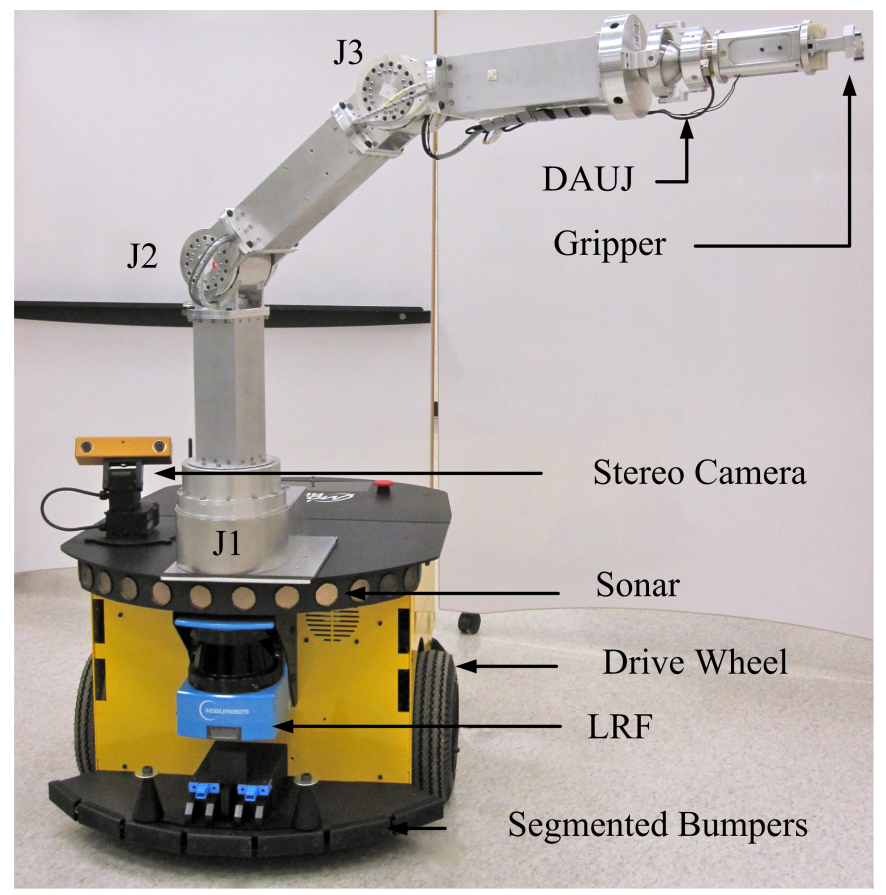

Figure 4.2: 5-DOF Mobile modular and reconfigurable robot.

mechanism (Ryew and Choi 2001). The motion range of each MRR module is as follows: joint 1: $\pm 180^{\circ}$, joints 2 and $3: \pm 120^{\circ}$, DAUJ (Wrist): $\pm 30^{\circ}$ pitch and yaw.

\subsubsection{Locomotion Base}

The locomotion system shown in Fig. 4.2 is based on the PowerBot Automated Guided Vehicle (AGV) from MobileRobots Inc. PowerBot has multiple sensors including a SICK laser rangefinder, a Bumblebee2 stereovision camera with a Pan-Tilt, two arrays of sonar sensors, and bumper sensors. The PowerBot is also equipped with a full-sized onboard PC used for the control of the mobile MRR. The robot platform is managed by an onboard microcontroller as well as mobile-robot server software, and consists of a balanced-drive system (two-wheel differential with balancing casters), motor-control and drive electronics, and high resolution motion encoders.

\subsection{Door Opening}

In the proposed door opening method, after the MRR has grasped and rotated the door knob, multiple working mode control is used to slightly open the door using only the MRR. During 
this process, the initial position of the mobile base, the door radius, and the height of the doorknob are estimated using the proposed parameter estimation algorithm. These estimated parameters are then used for planning the path of the mobile base that will allow opening the door to the desired angle. Finally, the multiple working mode control approach is used to control the MRR to complete the door opening process while preventing the occurrence of large internal forces.

The rest of this section is organized as follows: the first subsection describes the proposed door opening method, followed by a subsection that covers estimation of the door parameters, and the path planning of the mobile base is presented in the last subsection.

\subsubsection{Door Opening Method}

The door opening method is proposed with the following assumptions: i) the door axis of rotation is perpendicular to the floor, i.e., the door knob moves in the horizontal $x-y$ plane; this assumption always holds true unless a catastrophe like an earthquake has wrecked the architecture, a scenario beyond the scope of this thesis; ii) the mobile base travels on almost flat ground, which can always be satisfied for a structured indoor environment; iii) the axis of rotation of the first MRR module (turret) is perpendicular to the ground, which can be guaranteed by the mechanical design. The basic idea of the proposed door opening method is to utilize the multiple working modes of the MRR joint modules, to allow the door motion to guide the motion of the MRR, so as to avoid the generation of large internal forces. That is achieved by setting the axis of rotation of two joint modules parallel to the door hinge, then switching these joints to work in passive mode during the door opening process. How to set a joint in the passive working mode will be briefed later in subsection (4.5.2). For the aforementioned MRR, the axis of rotation of the first joint and that of the fourth joint, part of the DAUJ, are parallel to the door hinge in the nominal case. To make the axis of rotation of the fourth joint parallel to the door hinge, the second and third joints are set to their desired angles, which will be detailed in the door parameters estimation subsection. The 2-DOF motions of the DAUJ are coupled and cannot be set to work in passive mode independently, thus the fifth joint is also set in passive mode. The passivity of the 2-DOF DAUJ can compensate for any uncertainty in the active joint positions and/or any misalignment of the axes of rotation of the passive joints and that of the door. The passive joints act like free joints that are passively controlled by the coupling forces of the manipulator, thus their motion during the door opening process is directed by the motion of the door itself. The proposed door opening method can prevent the occurrence of internal forces, as the passive joints can absorb the position errors of the mobile manipulator while following the trajectory of the door knob. The movement of the 
mobile base along the planned trajectory generates the needed force for pulling the door open. The proposed door opening method is summarized as follows:

1. Grasp the door knob;

2. Estimate the door parameters and the initial position of mobile base as described in the door parameters estimation subsection;

3. Calculate the desired path of the mobile base using the estimated parameters;

4. Switch the first joint $J_{1}$ and the DAUJ $J_{4}$ and $J_{5}$ to work in passive mode;

5. Command the second and third joints to their desired angles calculated from the parameter estimation algorithm; and

6. Move the robot base along the predefined trajectory to pull the door open.

A comparison between the traditional methods and the proposed method is shown in Fig. 4.3 . The proposed method utilizes multiple working modes feature of the MRR joint modules. It does not require computationally heavy force relaxation algorithms. On the same basis, there is no need for tactile sensors at the gripper fingertips or any special mechanical design to perform the door opening task. In addition, the MRR configuration stays almost unchanged, as only the passive joints are moving throughout the door opening task. This minimizes the power consumption, which is especially important because the robot arm is running on the mobile base batteries.

\subsubsection{Door Parameters Estimation}

The mobile base motion during the door opening process has to be planned such that all joints move within their mechanical limits. In order to plan a safe path for the mobile base, the door parameters as well as the mobile base initial position, $\left(x_{1}, y_{1}\right)$, have to be estimated. The parameters needed to complete the door opening task are the door radius, $r$, and the knob height with respect to the base of the MRR, $h$. Here, we employ the method of least squares estimation to solve this problem. The idea is to estimate the door parameters and the initial mobile base position by using a recorded motion of the MRR end-effector. Fig. 4.4 shows the initial configuration of the mobile MRR, where $J_{i}$ denotes the $i$ th joint. The origin of the reference frame $\{G\}$ is set at the intersection point of the door hinge and the horizontal plane that crosses the origin of the reference frame of the MRR $\{O\}$. After the MRR end-effector has firmly grasped the door knob, we then, in the estimation process, apply a small torque only to

the $2^{\text {nd }}$ joint to slightly open the door. At the same time, all other joints are set in the passive 


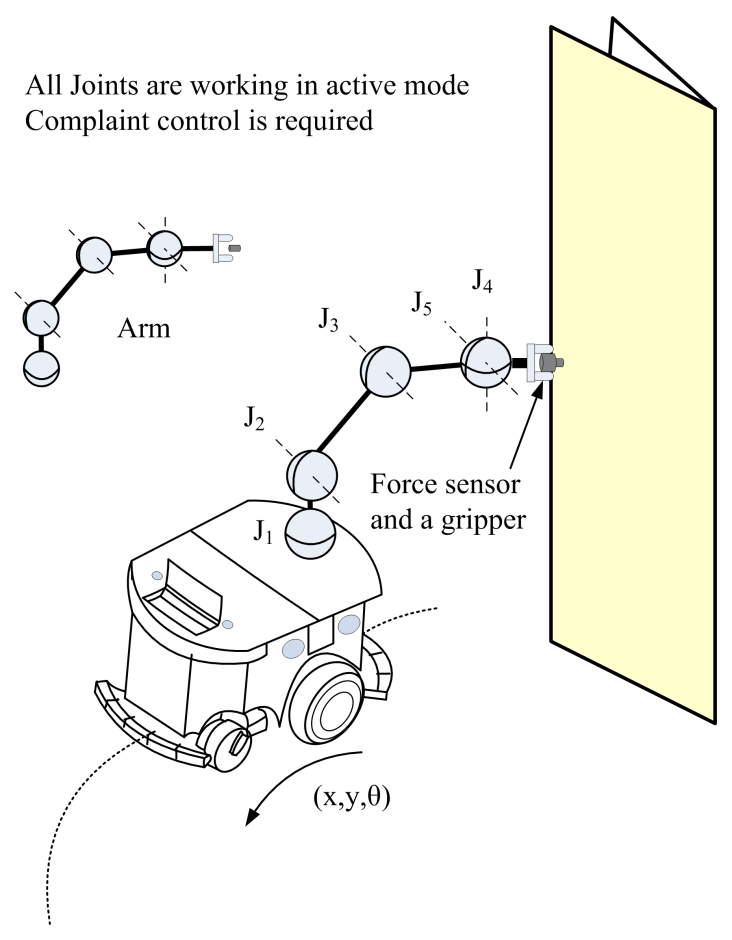

(a) Traditional method

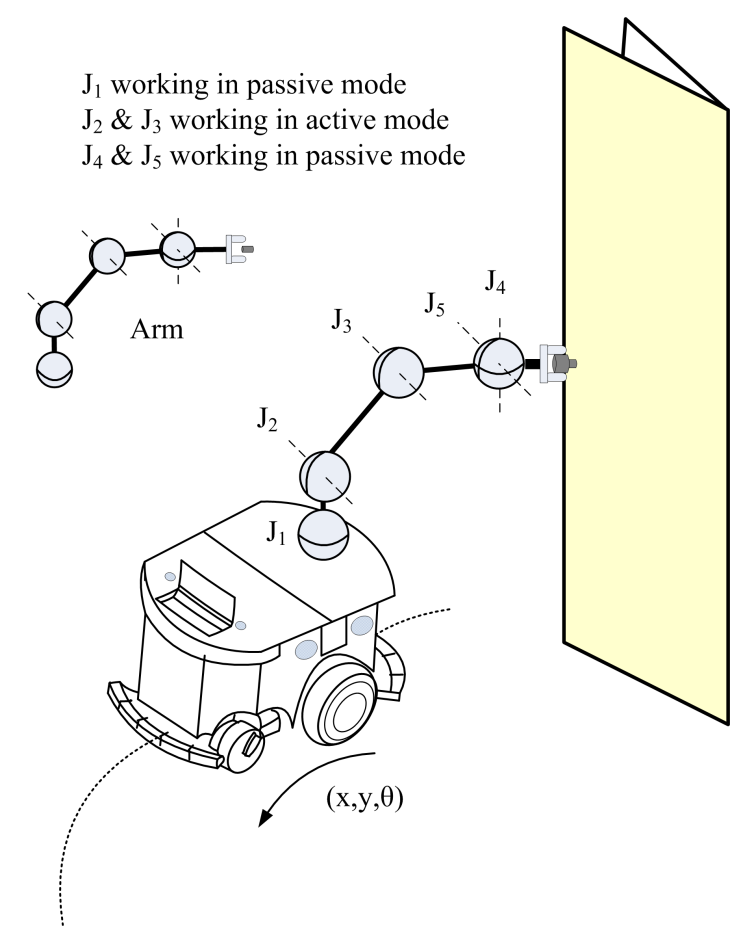

(b) Proposed method

Figure 4.3: Comparison between traditional and proposed door opening method. 


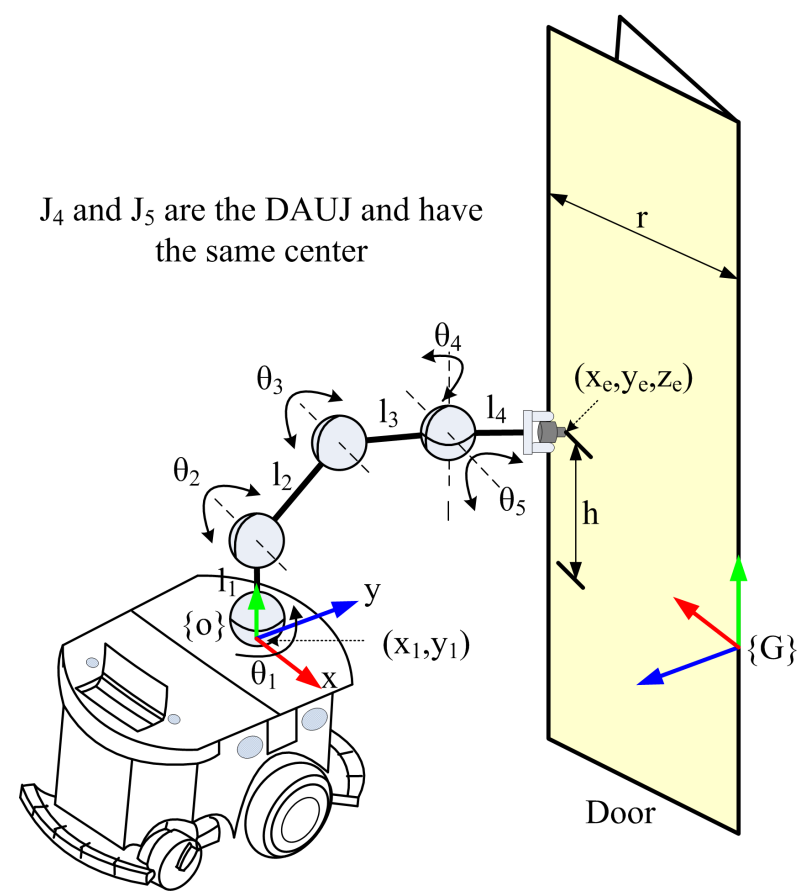

Figure 4.4: Initial configuration of the mobile MRR.

working mode. The MRR end-effector positions are recorded during this process. The saved data are then used to estimate the unknown door parameters as well as the current position of the mobile base. Let $\left(x_{e}, y_{e}, z_{e}\right)$ denote the tip position of the end-effector in the reference frame, $l_{i}$ denote the length of the $i$ th link, $\theta_{i}$ denote the rotation angle of the $i$ th joint, $C_{i}(t)$, $S_{i}(t), C_{i j}(t), S_{i j}(t)$ denote $\cos \left(\theta_{i}(t)\right), \sin \left(\theta_{i}(t)\right), \cos \left(\theta_{i}(t)+\theta_{j}(t)\right), \sin \left(\theta_{i}(t)+\theta_{j}(t)\right)$, respectively. From Fig. 4.4, with respect to the door radius, $r$ we have:

$$
\begin{gathered}
x_{e}^{2}(t)+y_{e}^{2}(t)=r^{2} \\
z_{e}(t)=h
\end{gathered}
$$

With respect to the reference frame $\{G\}$ shown in Fig. 4.4 and coordinate transfer, we can derive the following expression:

$$
\begin{aligned}
x_{e}(t) & =x_{1}+l_{4} C_{5}(t)\left(C_{1}(t) S_{4}(t)+S_{1}(t) C_{4}(t) C_{23}(t)\right)+l_{2} S_{1}(t) C_{2}(t) \\
& +l_{3} S_{1}(t) C_{23}(t)-l_{4} S_{1}(t) S_{5}(t) S_{23}(t)
\end{aligned}
$$




$$
\begin{aligned}
y_{e}(t) & =y_{1}+l_{4} C_{5}(t)\left(S_{1}(t) S_{4}(t)-C_{1}(t) C_{4}(t) C_{23}(t)\right)-l_{2} C_{1}(t) C_{2}(t) \\
& -l_{3} C_{1}(t) C_{23}(t)+l_{4} C_{1}(t) S_{5}(t) S_{23}(t) \\
z_{e}(t) & =l_{1}+l_{4} S_{5}(t) C_{23}(t)+l_{4} C_{4}(t) C_{5}(t) S_{23}(t)+l_{2} S_{2}(t)+l_{3} S_{23}(t)
\end{aligned}
$$

For simplicity, let us introduce the two function definitions $L_{x}(t)$ and $L_{y}(t)$, which are functions of explicitly known parameters only,

$$
\begin{aligned}
L_{x}(t) & \triangleq l_{4} C_{5}(t)\left(C_{1}(t) S_{4}(t)+S_{1}(t) C_{4}(t) C_{23}(t)\right)+l_{2} S_{1}(t) C_{2}(t)+l_{3} S_{1}(t) C_{23}(t) \\
& -l_{4} S_{1}(t) S_{5}(t) S_{23}(t) \\
L_{y}(t) & \triangleq l_{4} C_{5}(t)\left(S_{1}(t) S_{4}(t)-C_{1}(t) C_{4}(t) C_{23}(t)\right)-l_{2} C_{1}(t) C_{2}(t)-l_{3} C_{1}(t) C_{23}(t) \\
& +l_{4} C_{1}(t) S_{5}(t) S_{23}(t)
\end{aligned}
$$

By substituting $L_{x}(t), L_{y}(t)$ into (4.3), 4.4 and the resultant equations into 4.1) and rearranging each term, one has:

$$
L_{x}^{2}(t)+L_{y}^{2}(t)=r^{2}-x_{1}^{2}-y_{1}^{2}-2 x_{1} L_{x}(t)-2 y_{1} L_{y}(t)
$$

Define

$$
P=\left[\begin{array}{ccc}
1 & 2 L_{x}\left(t_{1}\right) & 2 L_{y}\left(t_{1}\right) \\
\vdots & \vdots & \vdots \\
1 & 2 L_{x}\left(t_{n}\right) & 2 L_{y}\left(t_{n}\right)
\end{array}\right], W=\left[\begin{array}{c}
L_{x}^{2}\left(t_{1}\right)+L_{y}^{2}\left(t_{1}\right) \\
\vdots \\
L_{x}^{2}\left(t_{n}\right)+L_{y}^{2}\left(t_{n}\right)
\end{array}\right], \lambda=\left[\begin{array}{c}
r^{2}-x_{1}^{2}-y_{1}^{2} \\
-x_{1} \\
-y_{1}
\end{array}\right]
$$

Equation (4.6) can be rewritten as:

$$
W=P \lambda
$$

A straightforward least squares approximation is then performed as,

$$
\hat{\lambda}=\left(P^{T} P\right)^{-1} P^{T} W
$$

where $\hat{\lambda}$ is the least square estimate for the unknown parameters. The axis of rotation of the MRR fourth joint shown in Fig. 4.4 needs to be parallel to the door hinge, as was explained in the door opening method subsection. This can be easily done by setting the second and third joint angles to their desired values calculated as follows. Once $h$ is known, the desired rotation 
angle of the $2^{\text {nd }}$ joint can be calculated from,

$$
\theta_{2 d}=\sin ^{-1}\left(\frac{h-l_{1}}{l_{2}}\right)
$$

The rotation angle of the third joint is calculated from the inverse kinematics of the MRR, $\theta_{3 d}=-\theta_{2 d}$. At that moment, the axis of rotation of the first and fourth joint is parallel to the door hinge. The other estimated parameters, i.e. the door radius and the mobile base initial position, are used for completing the path planning part.

\section{Limitations of the least squares estimation method}

One of the main disadvantages of least squares method is that when the data used for the estimation are corrupted by additive noise, the least square estimation method gives biased results. In addition, neglecting the presence of noise in general leads to models that do not characterize properly the underlying processes. In the literature, several modified least square methods have been proposed to estimate unknown parameters of system in the presence of noise, i.e., (Söderström 1981). Other limitations of using least squares methods for parameter estimation are the outliers and the limitations in the shapes that linear models can assume over long ranges, possibly poor extrapolation properties, and sensitivity to outliers.

The data used in our parameter estimation algorithm are the joint angles of the MRR robot which are measured with digital incremental encoders. Therefore, the presence of noise is assumed negligible. In addition, as the MRR is firmly grasping the door knob, outliers does not occur in the recorded data.

\subsubsection{Path Planning}

There are constraints that have to be considered when planning the trajectory of the mobile base. These constraints can be categorized into kinematic constraints, mechanical constraints and geometrical constraints. The kinematic constraints are due to the constrained motion of the robot that leads to a reduced number of degrees of freedom. The mechanical constraints are related to the mechanical structure of the robot, e.g., joint limits, maximum speeds, and maximum applicable torque and force at each joint module. Geometrical constraints exist between the door and the mobile base and collision has to be avoided throughout the door opening process. The objective of this subsection is to develop a path planning algorithm for the door opening task that takes the constraints imposed on the mobile MRR into consideration. The implementation of Hermite cubic splines as a tool for path planning is adopted to achieve this objective. Let $p=p_{1}, p_{2}, \ldots, p_{n}$ denote a set of control points (knots) on a route, with 
each point $p_{i}$ comprising of three basic elements, $x_{i}, y_{i}$ and $\theta_{i}, i=1,2, \ldots, n ; x_{i}, y_{i}$ denotes the position relative to the global reference frame, and $\theta_{i}$ is the heading angle of the mobile robot. There are several available methods to connect these points in a curve, with the most effective one for this case being the piecewise cubic polynomials method, alternatively called the cubic splines, for its offered features like continuity in position, heading, curvature, velocity, and acceleration (Strang 1986). Considering just the local parameterization of the $i$ th cubic spline segment in only the $x$ direction, one has:

$$
X_{i}(t)=a_{i}(t-1)^{2}(2 t+1)+b_{i} t^{2}(3-2 t)+c_{i} t(t-1)^{2}-d_{i} t^{2}(1-t)
$$

where $X_{i}$ denotes the segment connecting the points $p_{i}$ and $p_{i+1}$ in the $x$ direction; $0 \leq t \leq 1$ denotes the relative motion time of each segment $i(i=1,2, \ldots, n-1$ segments); $n$ denotes the total number of control points used on the path, and $a_{i}, b_{i}, c_{i}$, and $d_{i}$ are the coefficients to be determined. The Hermite cubic polynomial 4.10 is selected such that it satisfies all four of the following boundary conditions (Imran and Reza 2009):

$$
\begin{aligned}
& X_{i}(0)=a_{i} ; \quad \frac{d X_{i}}{d t}(0)=c_{i} \\
& X_{i}(1)=b_{i} ; \quad \frac{d X_{i}}{d t}(1)=d_{i}
\end{aligned}
$$

The first two boundary conditions are used to respectively assign the initial position and velocity values, and the last two boundary conditions are used to assign the final position and velocity values. The following additional constraints must be imposed to ensure continuity between each polynomial.

$$
\begin{aligned}
& X_{i}(1)=p_{x_{i+1}} ; \quad X_{i}(1)=X_{i+1}(0) \\
& \frac{d X_{i}}{d t}(1)=\frac{d X_{i+1}}{d t}(0) ; \quad \frac{d^{2} X_{i}}{d t^{2}}(1)=\frac{d^{2} X_{i+1}}{d t^{2}}(0)
\end{aligned}
$$

For $n$ control points, there are $4(n-1)$ equations and $4(n-1)$ unknowns. The system of equations (4.11) and 4.12 can be rewritten in a simple symmetric tridiagonal system of equations as follows:

$$
\left[\begin{array}{ccccc}
1 & & & & \\
1 & 4 & 1 & & \\
& \ddots & \ddots & \ddots & \\
& & 1 & 4 & 1 \\
& & & & 1
\end{array}\right]\left[\begin{array}{c}
\eta_{0} \\
\eta_{1} \\
\vdots \\
\eta_{n-2} \\
\eta_{n-1}
\end{array}\right]=\left[\begin{array}{c}
v_{\text {init }} \\
3\left(p_{x_{3}}-p_{x_{1}}\right) \\
\vdots \\
3\left(p_{x_{n-1}}-p_{x_{n-3}}\right) \\
v_{\text {final }}
\end{array}\right]
$$


where $\eta$ is an intermediate variable; $v_{\text {init }}, v_{\text {final }}$ are the initial and final velocities of the path, respectively. The coefficients of the spline are obtained by solving for the intermediate variables $\eta^{\prime} s$.

$$
a_{i}=p_{x_{i}} ; \quad b_{i}=p_{x_{i+1}} ; \quad c_{i}=\eta_{i-1} ; \quad d_{i}=\eta_{i}
$$

The initial position of the mobile base in the global reference frame was identified from the unknown parameter estimation algorithm while the final position is calculated using the desired door opening angle. First, we introduce an intermediate point between the known start and final position of the mobile base. Afterwards, a Hermite cubic polynomial is used to connect these points to create a rudimentary path. Finally, this created path is validated with respect to the constraint imposed on the system. These constraints and the margin in which the mobile robot can move are studied through computer simulation. For each door opening angle, the position of the mobile base was varied and an algorithm that checks the joint limits and other constraints was used to verify if that position is valid. The algorithm is described as follows:

1. For the current door opening angle starting from 0 deg;

(a) Calculate the door knob position;

(b) For the current mobile base position starting from the initial position, solve the inverse kinematics of the MRR considering the joint limits and check if the door knob is in the robot workspace;

(c) If (b) holds, using the mobile base footprint, calculate the distance between the door and the mobile base to verify that there is no possible collision;

(d) If (b) and (c) hold, save the mobile base position as a valid position.

2. Change the mobile base position by adding small $\Delta x$ and $\Delta y$;

3. Go to step (1) until one or more of the conditions is no longer satisfied;

4. Increase the door opening angle and repeat (1) to (3).

Using the aforesaid algorithm, the margin shown in Fig. 4.5 was obtained. Mobile base motion inside this margin will not violate any of the constraints and hence is allowed. We select the desired path to be in the middle of this margin to guarantee that all constraints are satisfied even in the case of large position errors of the mobile base. 


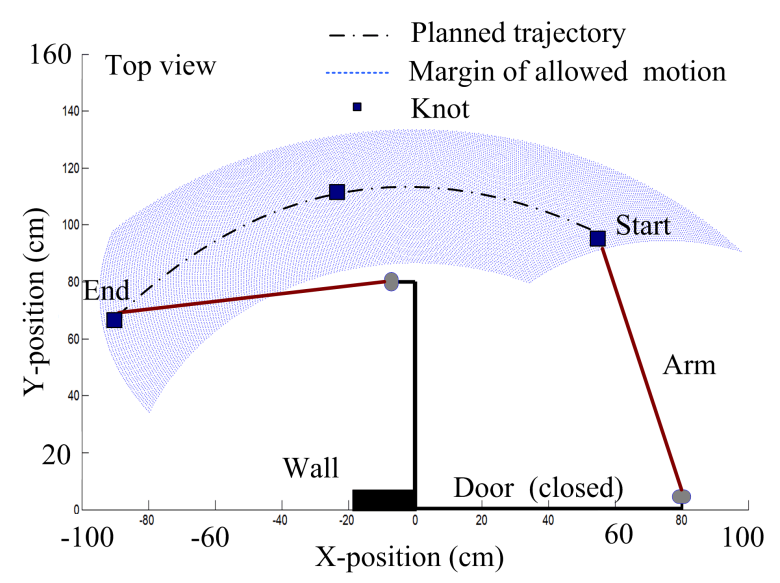

Figure 4.5: Trajectory of the mobile base and the margin of allowed motion.

\subsection{Simulation and Experiment Results}

\subsubsection{Simulation}

A series of computer simulations were carried out to validate the proposed door opening method and to ensure that all constraints are respected throughout the door opening process. The mobile base was assumed to have up to $10 \mathrm{~cm}$ error in Cartesian space when trying to follow a desired curvature trajectory. This desired trajectory was chosen to be in the middle of the mobile base safe motion margin. The results of some of these simulations are presented in Fig. 4.6 and Fig. 4.7 with $2 \mathrm{~cm}$ and $10 \mathrm{~cm}$ trajectory following errors, respectively. Simulations show that all joints move within their mechanical limits and there is no constraints violation even when the error in Cartesian space is up to $10 \mathrm{~cm}$.

\subsubsection{Experiments}

A door simulator with adjustable radius, knob height, and opening force is used to perform the door opening experiment; see Fig. 4.8. The opening force is adjusted by tightening or releasing a clamp on the door simulator. The knob position and the opening force are selected based on standard door dimensions and opening force. Positioning of the mobile base in front of the door simulator as well as the grasping of door knob is done manually. The desired path of the mobile base is calculated by the onboard computer using the estimated door parameters and the mobile base's initial position. Several experiments were carried out to confirm the efficiency of the proposed door opening method, all of which have been successful. The opening 


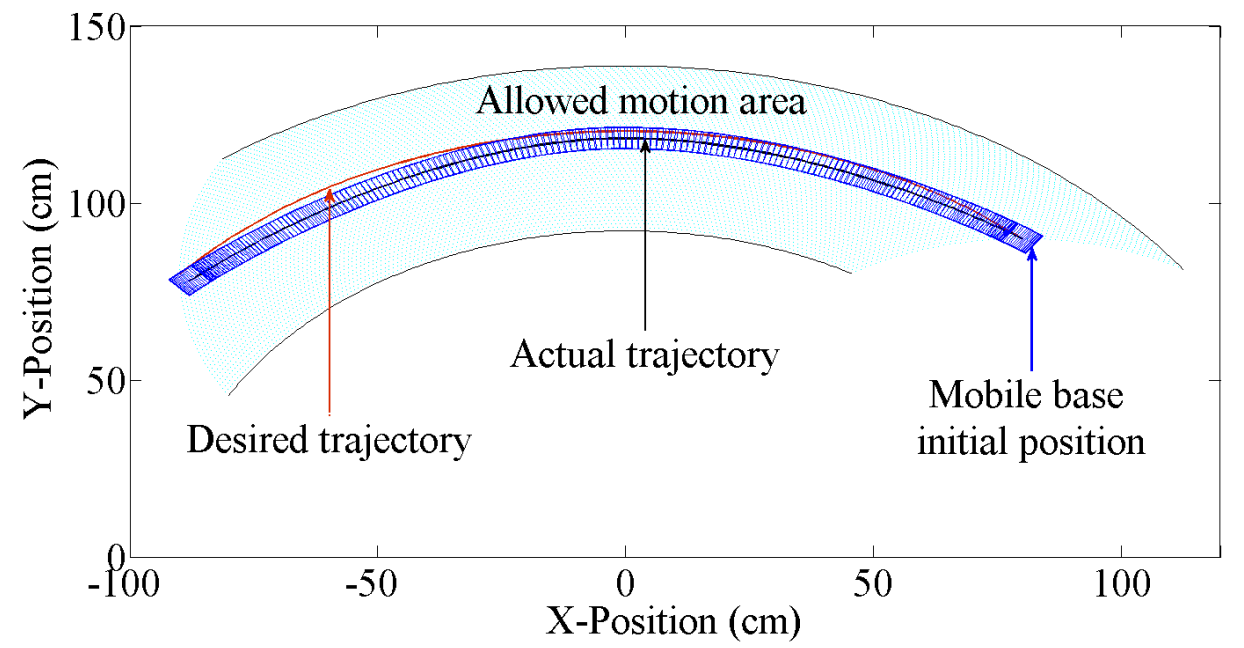

(a) Mobile base trajectory with $2 \mathrm{~cm}$ position error. The light-blue envelope represents the allowed motion area.

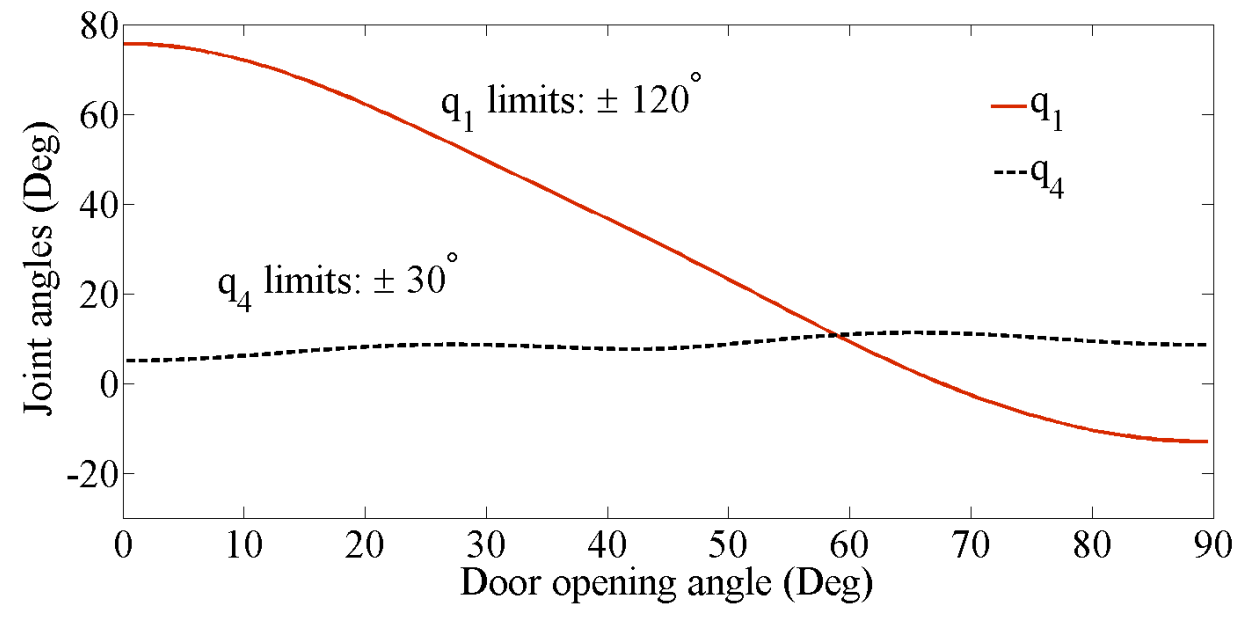

(b) The first and fourth joint angles.

Figure 4.6: Simulation results for $2 \mathrm{~cm}$ position error. 


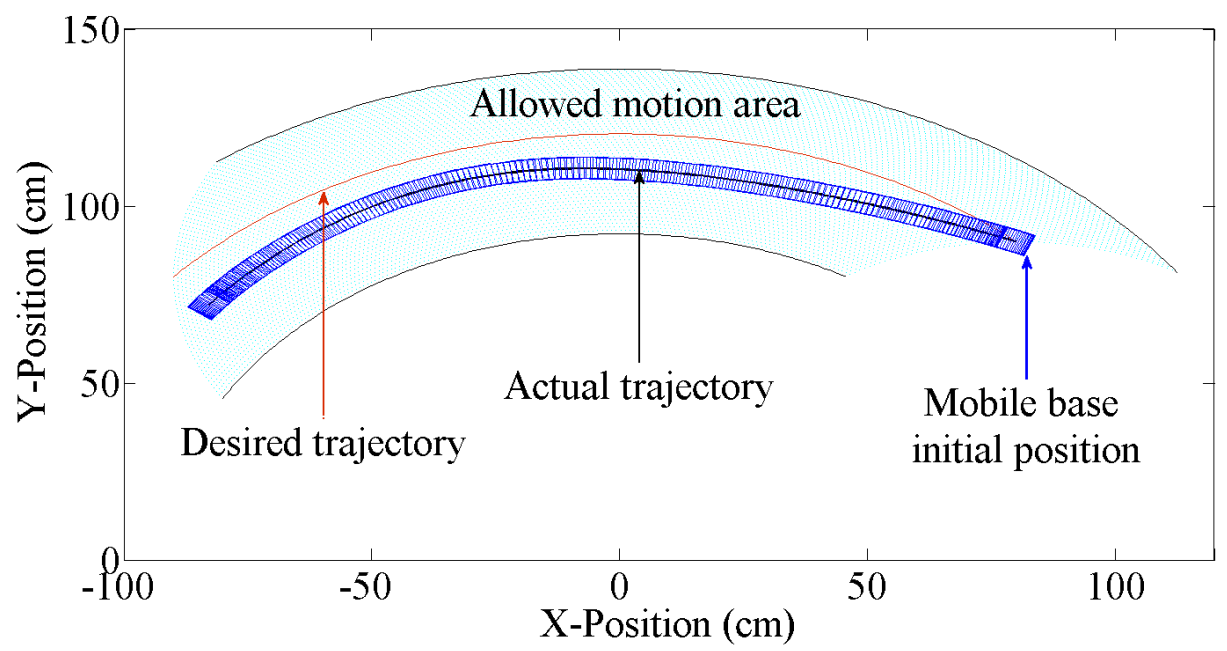

(a) Mobile base trajectory with $10 \mathrm{~cm}$ position error. The light-blue envelope represents the allowed motion area.

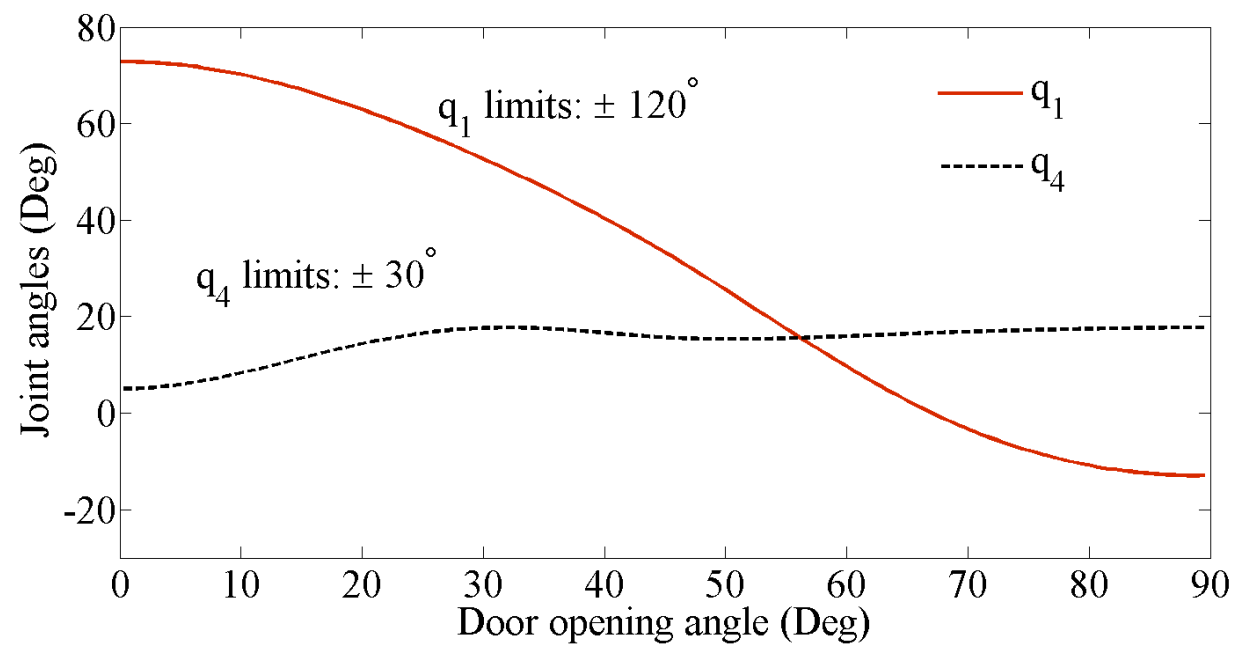

(b) The first and fourth joint angles.

Figure 4.7: Simulation results for $10 \mathrm{~cm}$ position error. 


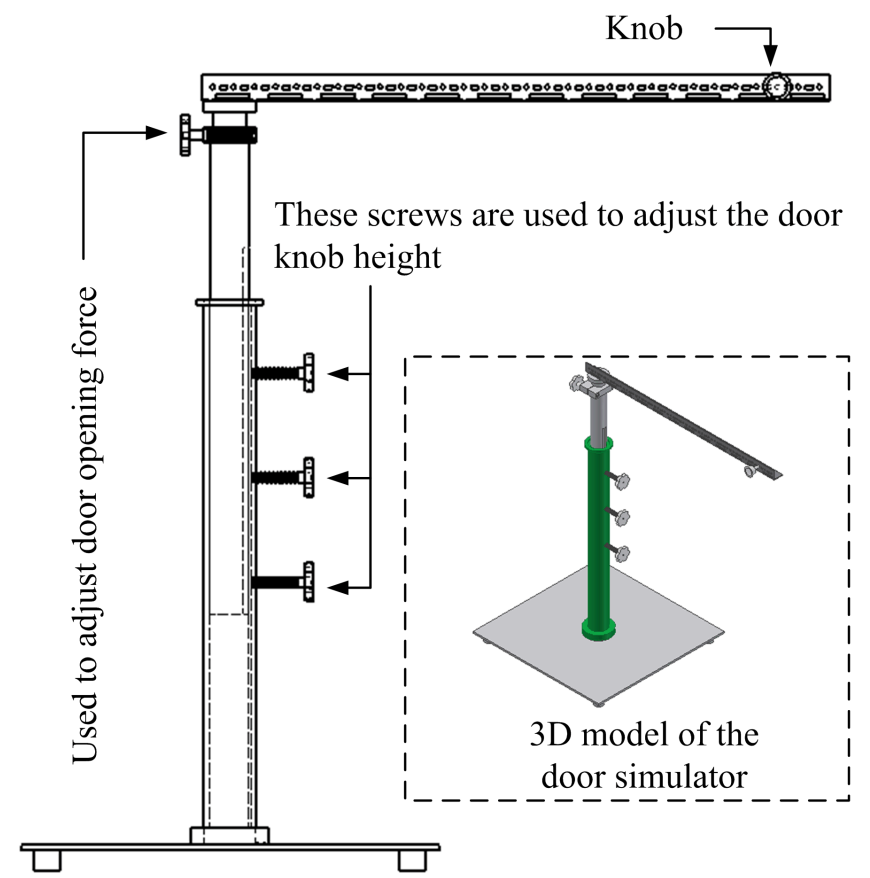

Figure 4.8: Schematic diagram of the door simulator.

of door is shown by sequential pictures in Fig. 4.9. In Figure 4.9, picture (a) shows the start position; (b) shows that the mobile base has started following the desired path with MRR joints 1, 4 and 5 working in passive mode; (c), (d), and (e) show the yaw angle of the DAUJ has changed (see Fig. 4.11 for the yaw angle and the corresponding motion time); (f) shows that the door is fully open, $90^{\circ}$. The joint positions and the readings of the integrated torque sensor of each MRR module as well as the six-axis force sensor were recorded during the door opening process. Fig. 4.10 shows the force measurements of the force sensor along the $x, y$, and the $z$ directions. The force in the $z$ direction, $F z$, represents the pulling force, and is expected to be large due to the force applied by the door in this direction. The other two forces, $F x$ and Fy, represent the force in the pitch and yaw directions of the DAUJ, respectively. The torques generated by these forces in the pitch and yaw direction of the DAUJ and the DAUJ joint position angles are shown in Fig. 4.11. The torque sensor readings of the MRR $J_{1}$ and its position angle are shown in Fig. 4.12. It is clear from the experimental results that the multiple working mode control can prevent the occurrence of large internal forces during the door opening process. The torque measurements at the wrist were less than $2.3 \mathrm{Nm}$. It is worth noting that the DAUJ has a threshold of around $2 \mathrm{Nm}$ before it starts moving when working in the passive mode. This is partially due to the uncompensated part of the joint friction. This 


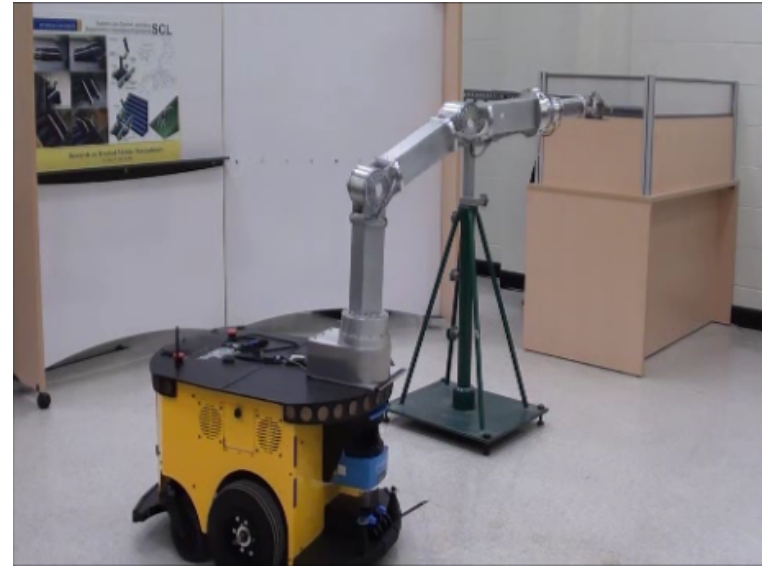

(a)

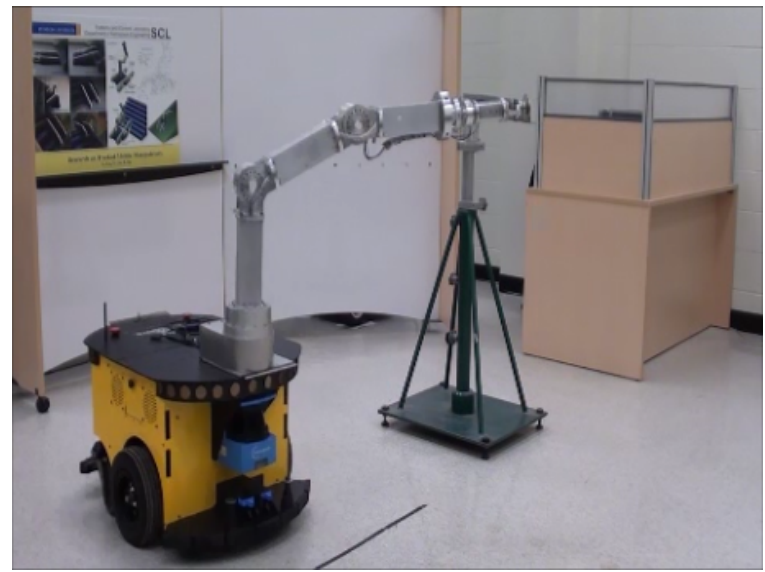

(c)

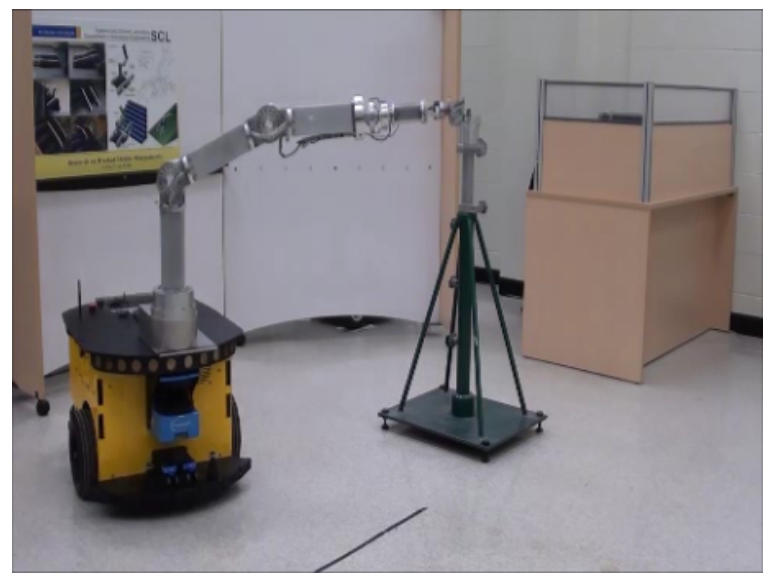

(e)

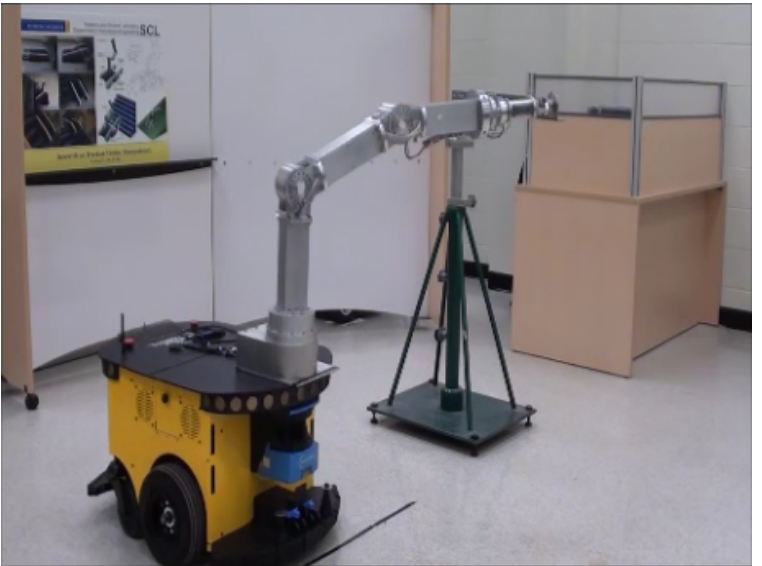

(b)

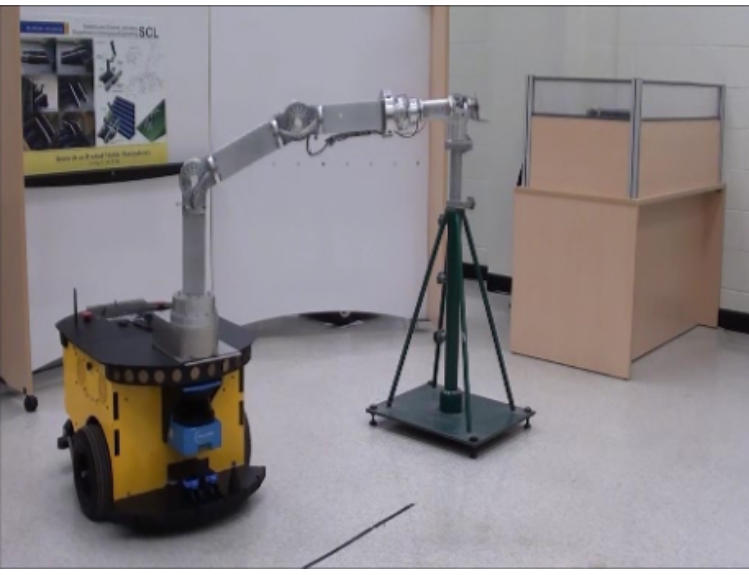

(d)

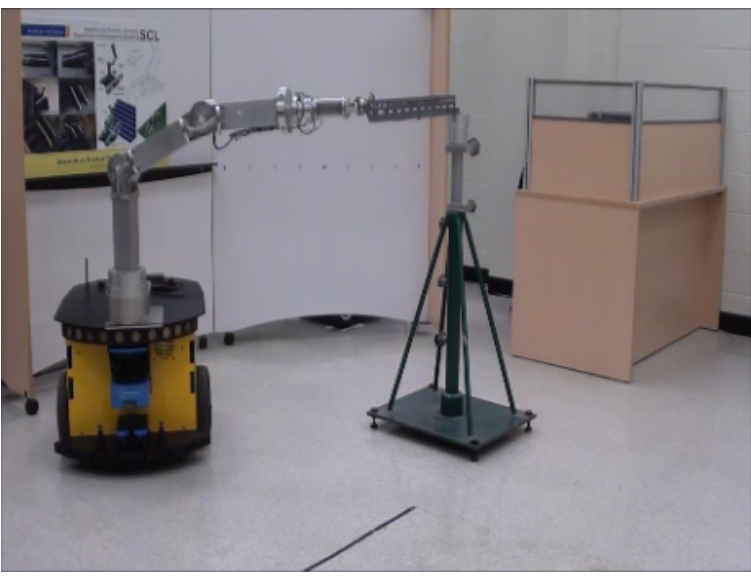

(f)

Figure 4.9: Pictures of the mobile MRR pulling door open. 


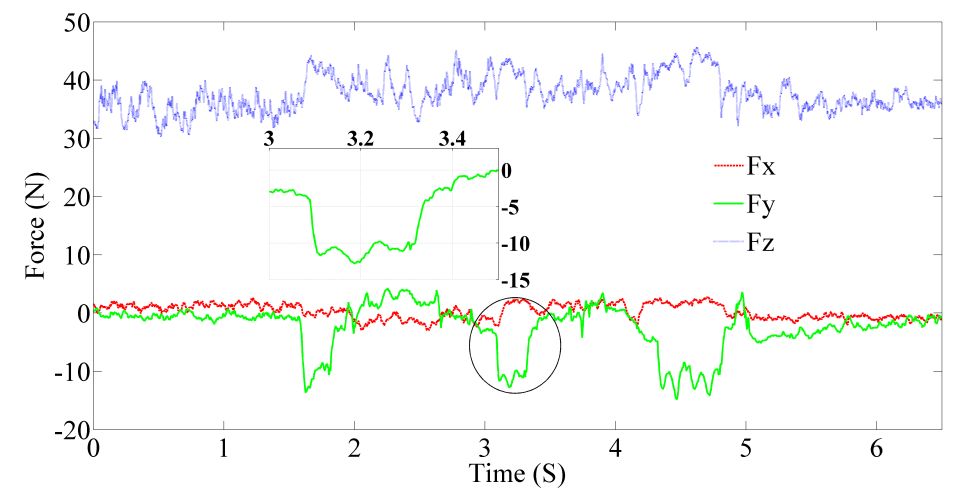

Figure 4.10: Force measurements at the MRR end-effector.

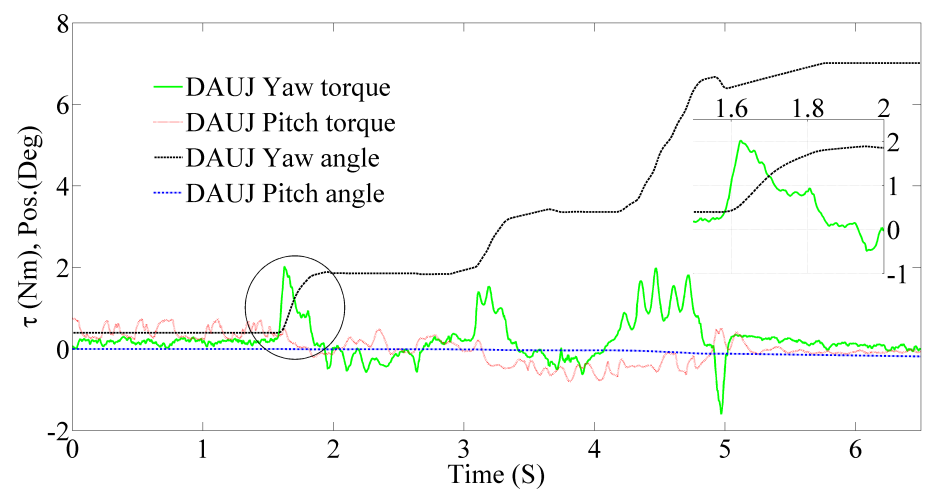

Figure 4.11: DAUJ pitch and yaw positions and torques.

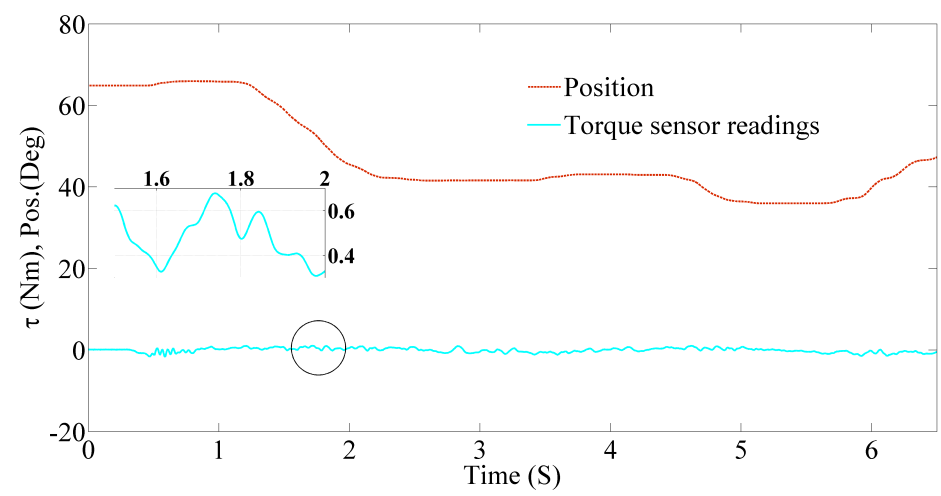

Figure 4.12: Torque sensor readings and joint angle of the MRR joint 1. 


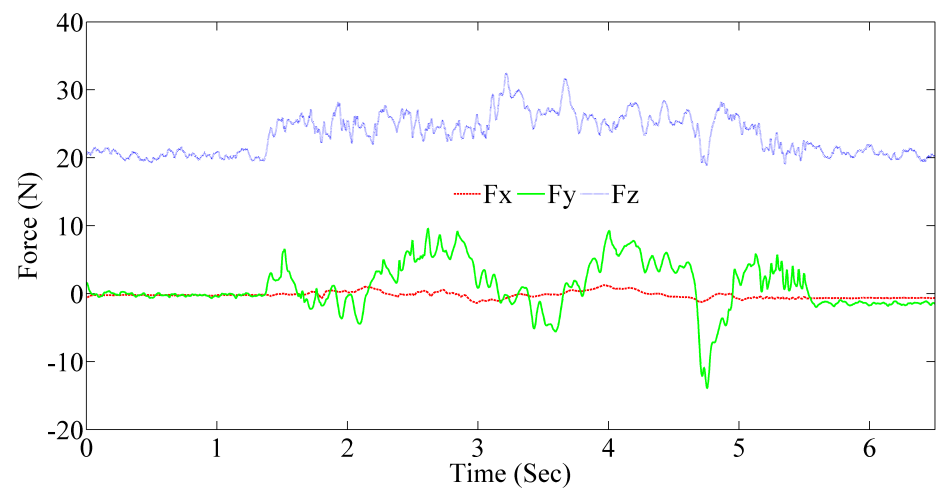

Figure 4.13: End-effector forces with $2^{o}$ heading angle error.

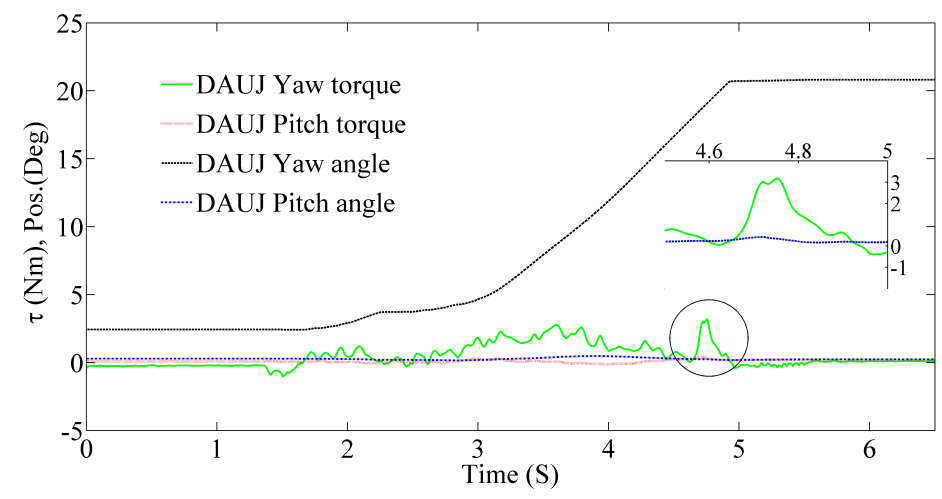

Figure 4.14: DAUJ angles and torques with $2^{\circ}$ heading angle error.

threshold is necessary to prevent DAUJ motion in absence of external forces as a result of the gripper weight and it has been selected experimentally by trial and error. This implies that the measured internal forces are less than $0.3 \mathrm{Nm}$. In comparison to the previously proposed door opening methods, these internal forces are of similar magnitude to those achieved when using complicated compliant control algorithms. Experiments also showed that the proposed method is robust to the accumulated position errors of the mobile base. This was confirmed by repeating the experiment with different initial heading angles of the mobile base to change its trajectory while making sure it was still within the allowed margin. The mobile MRR could open the door with measured internal forces that are still of comparable magnitude with the case in which no heading error is introduced. For example, the torque measurements at the wrist reached a maximum of $3 \mathrm{Nm}$ when offsetting the mobile base initial heading angle by 2 degrees. The results of the door opening experiment when mobile base heading angle error is introduced are shown in Fig. 4.13 to Fig. 4.15. The torque measurements at the wrist, as 


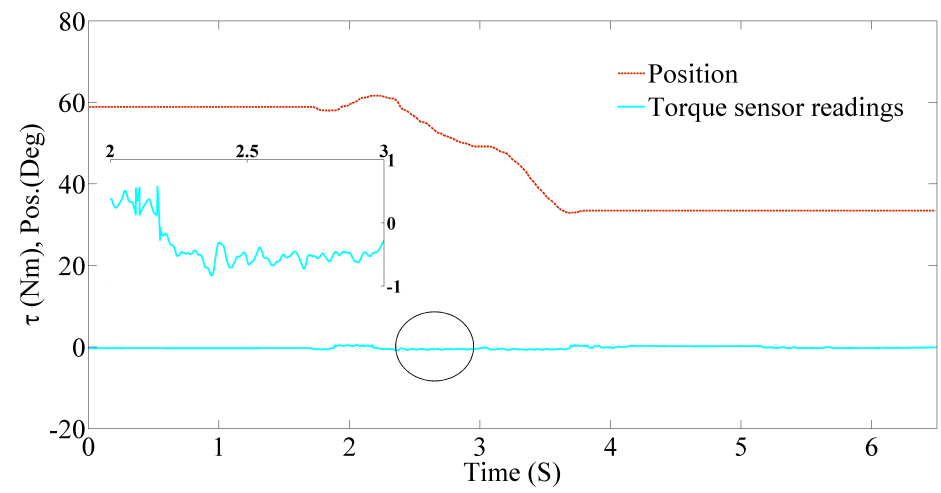

Figure 4.15: Joint 1 angle and torque sensor readings with $2^{\circ}$ heading angle error.

shown in Fig. 4.14, are similar to those of the previous experiment. During the experiments, a minor relative motion between the robot end-effector and the door knob was noticed. This minor relative motion did not affect the door opening performance because the free joints are passively controlled by the coupling forces between the MRR and the door, and these forces are independent of the contact point.

\subsection{Concluding Remarks}

In this chapter, a novel door opening method is presented along with its experimental demonstration using a mobile MRR. By switching between active and passive working modes of the MRR joint modules, the occurrence of large internal forces was prevented. The presented method solves a major problem of door opening without using complicated control algorithms or any special mechanical design, and it can be implemented on any other robot that has joints capable of working in both active and passive modes. 


\section{Chapter 5}

\section{Distributed Fault Detection for Modular and Reconfigurable Robots with Joint Torque Sensing: A Prediction Error Based Approach}

\subsection{Introduction}

In many modern applications require robots to operate in unstructured environments where human intervention may not be feasible due to cost or environmental dangers. Robot reliability and fault tolerance are critical for successful operations in such environments. For robots working alongside human beings, like medical robots for example, timely detection of robot faults is more of a concern. It is therefore essential for robots to independently detect and isolate internal failures and, if possible, utilize the remaining functional capabilities to overcome the limitations forced by the failures and safely complete their tasks.

Fault detection and fault tolerant control have been attracting a great deal of interest in the control engineering and robotics communities as it enables systems to continue operation, possibly at a reduced level, when some part of the system fails (Liu 2001; Abdul and Liu 2007; Abdul and Liu 2008; Paviglianiti et al. 2010). The main objective of fault detection and isolation is to quickly detect faults and provide early warnings to facilitate appropriate preventive actions so that complete system breakdown can be avoided. In this chapter, a distributed fault detection scheme is developed for modular and reconfigurable robots with joint torque sensing. The fundamental idea is to compare the filtered motor torque commands 
of monitored MRR with filtered torque estimates derived from its distributed dynamical model. Fault is declared if the residual of this comparison goes above certain threshold.

There are several fault detection methods in the existing literature for robot manipulators, e.g., (Brambilla et al. 2008; Yuan et al. 2011; Zhang 2011). In (Brambilla et al. 2008), a fault detection and isolation scheme based on the inverse dynamics approach is presented for robot manipulators. In this work, a suboptimal second-order sliding-mode algorithm is used to design the input laws of the observers. In (Yuan et al. 2011), joint torque measurement is used to estimate the power efficiency of MRR's joint, and residuals are generated by comparing the input and the output power of each MRR joint module. A fault detection scheme with online estimators is investigated in (Zhang 2011) to estimate the unknown constant sensor bias for diagnosis of sensor faults. In (Shin and Lee 1999), the position and velocity tracking errors are used to detect joint failures; a failure is declared if a selected combination of the position error and velocity error exceeds a predefined threshold. For a specific trajectory a threshold can be defined through some online tuning. However, a globally efficient error threshold may not be feasible due to a variety of reasons that may include differences in the type of tracking control law and its parameters, the payload variation, the position and orientation of the robot manipulator, etc. A poorly designed error threshold may lead to false alarms. In (Caccavale and Walker 1997), residuals are generated by comparing predicted observer outputs with measured system outputs. This observer-based scheme requires the measurement or estimation of accelerations. Other observer-based fault detection algorithms are also reported in (Schneider and Frank 1996. Chan et al. 1999; Izumikawa et al. 2005). A model-based fault detection approach that relies on generation of residuals through a filtered torque estimate is proposed in (Dixon et al. 2000; McIntyre et al. 2005). Analytical redundancy has been investigated in the literature to partially replace the hardware redundancy with the aim of reducing the overall cost and improving reliability (Frank et al. 2000; Hahn et al. 2003). Online parameter estimation method is used in (Freyermuth 1991) to monitor and identify sudden changes in system parameters due to faults in robotic systems. The simplification of the dynamic model by ignoring the coupling between joints in (Freyermuth 1991) necessitates the need for conservative thresholds to avoid false alarms. A neural-networks based fault detection algorithm was proposed in (Eski et al. 2011). A comprehensive overview of fault detection methods is presented in (Hwangand et al. 2010).

In this work, a distributed fault detection scheme is developed for modular and reconfigurable robots with joint torque sensing. This method calculates the difference between filtered motor torque commands and filtered torque estimates to generate residuals with which fault can be detected. Since the torque commands and the torque estimates are filtered, the measurement or estimation of the robot joint accelerations are not required. The proposed scheme 
takes into account uncertainties in system parameters, namely, the friction model parameters. The rotor inertia and gear ratio are assumed to be known accurately and are constant. The friction model parameters of each MRR joint are initially estimated using observer based techniques (Tien et al. 2008) and the resulting parameters are considered as nominal values. It is a well known fact that these parameters change over time. Therefore, an adaptive law is used to update the friction model parameters online as a means of improving the sensitivity of the proposed distributed fault detection scheme.

The actuator faults that are considered in this research work are of three types: free-swinging actuator fault, ramp actuator fault, and saturated actuator fault. The term free-swinging refers to a fault in an MRR joint module that might be caused by loss of actuator torque, loss of electrical power and brakes of an MRR joint, or a mechanical failure in the drive system. The ramp actuator fault and the saturated actuator fault represent a joint that is gradually drifting from the actual torque command and a suddenly saturated joint, respectively. The latter two faults may be attributed to damage in the motor windings, faulty electronics in the drive system, and/or sensors failures. Sensor failures refer to the motor hall sensors that are used to detect the rotor position and produce position signal for the gating control of the driver circuitry in the case of brushless DC motors.

The rest of this chapter is organized as follows: The dynamic model of modular and reconfigurable robot with joint torque sensing that may undergo actuator fault is described Section 5.2 . The proposed fault detection scheme is presented in Section (5.3). Experimental results are presented and discussed in Section (5.4), and concluding remarks are given in Section (5.5).

\subsection{Dynamic Model of the MRR with Consideration of Faults}

With consideration of actuator faults, the dynamical equation (3.4) can be rewritten as:

$$
\begin{aligned}
& M_{i} \ddot{q}_{i}(t)+\delta_{i}(t)+\gamma_{i}^{-1} \tau_{s i}(t)+Y_{i}\left(\dot{q}_{i}(t), \tau_{s i}(t)\right) \theta_{i}+\Phi_{i}\left(\dot{q}_{i}(t)\right) \operatorname{sign}\left(\dot{q}_{i}(t)\right) \\
& +u_{-1}\left(t-T_{f}\right) \zeta_{i}(t)=\tau_{i}(t)
\end{aligned}
$$

where $\zeta_{i}(t) \in \Re$ represents a fault in the $i$ th MRR joint module, $u_{-1}\left(t-T_{f}\right)$ represents a unit step function, $T_{f}$ is the time instant when a fault occurs. The following property of dynamic equation (5.1) and the succeeding assumption are worth noting as they are used in the development of fault detection scheme.

Property 3 The accelerations and velocities of the lower stabilized and fault free joint modules 
are bounded (Abdul and Liu 2008), and therefore, the following upper bound exists:

$$
\left|\delta_{i}(t)\right| \leq \bar{\delta}_{i}
$$

Assumption 1 A controller is designed which ensures that in the absence of a fault (i.e., $\left.\forall t<T_{f}\right) q_{i}(t), \dot{q}_{i}(t), \tau_{i}(t) \in \mathcal{L}_{\infty}$ and that $\lim _{t \rightarrow \infty} q_{i}(t) \rightarrow q_{d i}(t)$ where $q_{d i}(t) \in \Re$ is the desired ith joint trajectory.

Note that based on the form of the dynamic model given in 5.1), if $q_{i}(t), \dot{q}_{i}(t), \tau_{i}(t) \in \mathcal{L}_{\infty}$ it is clear that $\ddot{q}_{i}(t) \in \mathcal{L}_{\infty}$.

In the presence of an actuator fault, the dynamic equation (5.1) can be rewritten as follows:

$$
\zeta_{i}(t)=\tau_{i}(t)-\left[M_{i} \ddot{q}_{i}(t)+N_{i}(t)\right] \quad \forall t \geq T_{f}
$$

where

$$
N_{i}(t) \triangleq \delta_{i}(t)+\gamma_{i}^{-1} \tau_{s i}(t)+Y_{i}\left(\dot{q}_{i}(t), \tau_{s i}(t)\right) \theta_{i}+\Phi_{i}\left(\dot{q}_{i}(t)\right) \operatorname{sign}\left(\dot{q}_{i}(t)\right)
$$

Similar to (McIntyre et al. 2004), the actuator faults considered in this thesis are categorized as follows: a free-swinging actuator fault (i.e., $\left[M_{i} \ddot{q}_{i}(t)+N_{i}(t)\right]=0$ ), a ramp actuator fault (i.e., $\left.\left[M_{i} \ddot{q}_{i}(t)+N_{i}(t)\right]=\vartheta_{i} t\right)$, and a saturated actuator fault (i.e., $\left.\left[M_{i} \ddot{q}_{i}(t)+N_{i}(t)\right]=\tau_{\max i}\right)$, is characterized as in (5.4).

$$
\zeta_{i}(t) \triangleq\left\{\begin{array}{l}
\tau_{i} \\
\tau_{i}-\vartheta_{i} t \quad \forall t \geq T_{f} \\
\tau_{i}-\tau_{\max i}
\end{array}\right.
$$

where, $\vartheta_{i} \in \Re$ is positive scaling term and $\tau_{\max i} \in \Re$ represents the maximum applicable torque at actuator $i$.

Remark 4 One method for detecting actuator faults could be by utilizing the following equation:

$$
\zeta_{i}(t)=\tau_{i}(t)-\left[M_{i} \ddot{q}_{i}(t)+N_{i}(t)\right]
$$

However, the method in 5.5 is deemed impractical for fault detection as it requires not just the knowledge of exact MRR model but also acceleration measurements. In addition, for the $i$ th joint, the motion states of all lower joint modules are required to construct the coupling effect term, which makes this approach unsuitable for modular robots. The objective of the present work is to design a distributed fault detection scheme that does not require acceleration measurements or motion states of other joints. 


\subsection{Distributed Prediction Error Based Fault Detection}

The proposed distributed fault detection scheme is described in this section. The torque command of the $i$ th joint module is filtered and compared with a filtered torque estimate that is derived from its dynamic equation given in (5.1). Driven by the wish of eliminating joint acceleration measurements from the fault detection scheme, a filtered torque signal denoted by, $\tau_{f i}(t) \in \Re$ is defined as follows:

$$
\tau_{f i}(t)=f(t) * \tau_{i}(t)
$$

where the symbol $*$ denotes the standard convolution operation, and the low-pass filter function $f(t)$ is given by:

$$
f(t)=\alpha e^{-\beta t}
$$

where $\alpha, \beta \in \Re$ are positive filter constants.

Remark 5 As a result of the filtering, the filtered fault signal is delayed from the actual fault, although the delay can be mitigated by selecting large value of $\beta$.

Equation (5.1) can also be rewritten in the following form:

$$
\tau_{i}=\dot{h}_{i}+\ell_{i}
$$

where

$$
\begin{gathered}
\dot{h}_{i}=M_{i} \frac{d}{d t} \dot{q}_{i}(t) \\
\ell_{i}=\delta_{i}(t)+\gamma_{i}^{-1} \tau_{s i}(t)+Y_{i}\left(\dot{q}_{i}(t), \tau_{s i}(t)\right) \theta_{i}+\Phi_{i}\left(\dot{q}_{i}(t)\right) \operatorname{sign}\left(\dot{q}_{i}(t)\right)+u_{-1}\left(t-T_{f}\right) \zeta_{i}(t)
\end{gathered}
$$

Using the fact that convolution is distributive, substituting (5.8) into (5.6) yields:

$$
\tau_{f i}(t)=f(t) * \dot{h}_{i}+f(t) * \ell_{i}
$$

Using standard convolution properties, one can write:

$$
f(t) * \dot{h}_{i}=\dot{f}(t) * h_{i}+f(0) h_{i}-f(t) h_{i}(0)
$$

By substituting (5.10) and the expressions for $h_{i}$ and $\ell_{i}$ into 5.9 , one obtains:

$$
\begin{aligned}
\tau_{f i}(t) & =\dot{f}(t) * M_{i} \dot{q}_{i}(t)+f(0) M_{i} \dot{q}_{i}(t)-f(t) M_{i} \dot{q}_{i}(0)+f(t) *\left\{\delta_{i}(t)+\gamma_{i}^{-1} \tau_{s i}(t)\right. \\
& \left.+Y_{i}\left(\dot{q}_{i}(t), \tau_{s i}(t)\right) \theta_{i}+\Phi_{i}\left(\dot{q}_{i}(t)\right) \operatorname{sign}\left(\dot{q}_{i}(t)\right)+u_{-1}\left(t-T_{f}\right) \zeta_{i}(t)\right\}
\end{aligned}
$$


Equation (5.11) can be rewritten as:

$$
\begin{aligned}
\tau_{f i}(t) & =\dot{f}(t) * M_{i} \dot{q}_{i}(t)+f(0) M_{i} \dot{q}_{i}(t)-f(t) M_{i} \dot{q}_{i}(0)+Y_{f i}\left(q(t), \dot{q}_{i}(t), \tau_{s i}(t)\right) \theta_{i} \\
& +\delta_{f i}(t)+f(t) *\left\{\gamma_{i}^{-1} \tau_{s i}(t)+\Phi_{i}\left(\dot{q}_{i}(t)\right) \operatorname{sign}\left(\dot{q}_{i}(t)\right)\right\}+\zeta_{f i}(t)
\end{aligned}
$$

where, $\delta_{f i}=f(t) * \delta_{i}, \theta_{i}$ is the same constant parameter vector as defined in 2.27), $Y_{f i}\left(q_{i}, \dot{q}_{i}, \tau_{s i}\right)$ denotes a measurable filtered regression vector and $\zeta_{f i}(t) \in \Re$ denotes the filtered fault signal defined as follows:

$$
\zeta_{f i}(t)=f(t) * u_{-1}\left(t-T_{f}\right) \zeta_{i}(t)
$$

Remark 6 Based on (5.12), it is clear that fault can be isolated in terms of an expression that is independent of link acceleration measurements. Note that $M_{i}$ is the rotor inertia multiplied by the gear ratio, the values of which are accurately known. $\tau_{s i}, q_{i}, \dot{q}_{i}, \Phi_{i}\left(\dot{q}_{i}(t)\right)$, and $Y_{f i}\left(q(t), \dot{q}_{i}(t), \tau_{s i}(t)\right)$ are also available; the nominal values, $\hat{\theta}_{i}$, can be substituted for $\theta_{i}$ and hence, the filtered fault signal can be calculated.

The following measurable prediction error is used to detect the fault at each joint module:

$$
\mathbf{e}_{i}(t)=\tau_{f i}(t)-\hat{\tau}_{f i}(t)
$$

where $\tau_{f i}(t)$ is defined in 5.12 , and $\hat{\tau}_{f i}(t)$ is the filtered torque estimate to be derived.

It also is clear from 5.12 that the only term that depends on motion states of other joint modules is $\delta_{f i}(t)$. This term represents the filtered coupling effect between the lower $i-1$ joints and the $i$ th joint which is a function of lower $i-1$ joints positions and velocities. For MRRs with joint torque sensing, the link dynamics are captured by integrated torque sensors, and $\delta_{f i}(t)$ is coupling in the motor dynamics only as can be seen from (3.5). If each coupling term is considered as a modeling error, then an adaptation law guaranteeing the convergence of filtered torque estimate to the actual filtered torque in the presence of nonzero modeling errors can be designed. At this point, to explain the structure of the proposed fault detection scheme, the filtered coupling term $\delta_{f i}(t)$ is obtained using the known motion state of the lower $i-1$ joints. The filtered torque estimate is designed using the known nominal values of system parameters as follows:

$$
\begin{aligned}
\hat{\tau}_{f i}(t) & =\dot{f}(t) * M_{i} \dot{q}_{i}(t)+f(0) M_{i} \dot{q}_{i}(t)-f(t) M_{i} \dot{q}_{i}(0)+Y_{f i}\left(q_{i}(t), \dot{q}_{i}(t), \tau_{s i}(t)\right) \hat{\theta}_{i} \\
& +\delta_{f i}(t)+f(t)\left\{\gamma_{i}^{-1} \tau_{s i}(t)+\Phi_{i}\left(\dot{q}_{i}(t)\right) \operatorname{sign}\left(\dot{q}_{i}\right)(t)\right\}
\end{aligned}
$$


Substituting (5.12) and (5.15) into (5.14), one obtains:

$$
\mathbf{e}_{i}(t)=-Y_{f i}\left(q_{i}(t), \dot{q}_{i}(t), \tau_{s i}(t)\right) \tilde{\theta}_{i}+\zeta_{f i}(t)
$$

where $\tilde{\theta}_{i}$ reflects the discrepancy between the actual and the nominal values of the MRR joint parameters and is given by:

$$
\tilde{\theta}_{i}=\hat{\theta}_{i}-\theta_{i}
$$

An upper bound on the prediction error in equation $(5.16)$ can be defined as follows:

$$
\left|\mathbf{e}_{i}\right| \leq \rho_{i}(t)+\left|\zeta_{f i}(t)\right|
$$

where $\rho_{i}(t) \in \Re$ is a positive bound that is selected to satisfy the following inequality:

$$
\left|Y_{f i}\left(q_{i}(t), \dot{q}_{i}(t), \tau_{s i}(t)\right) \tilde{\theta}_{i}\right| \leq \rho_{i}(t)
$$

Therefore, a dead-zone residual function can be defined to indicate a fault as follows:

$$
D\left(\mathbf{e}_{i}\right)=\left\{\begin{array}{ccc}
1 & \text { if } & \left|\mathbf{e}_{i}\right|>\rho_{i}(t) \\
0 & \text { if } & \left|\mathbf{e}_{i}\right| \leq \rho_{i}(t)
\end{array}\right.
$$

To construct a distributed fault detection scheme, the fault detection of each joint module should be independent of the motion state of any other joint module. This is important due to the fact that joint modules may be added or removed at any time to MRRs. Therefore, it desirable to have a fault detection scheme that does not depend on the motion states of other joint modules. In order to craft a sensitive fault detection scheme that does not require the motion state of other joint modules, an online estimator for the uncertain parameters, $\theta_{i}$, is designed while the coupling terms $\delta_{f i}(t)$ are treated as modeling errors. In this case, the filtered torque estimate is designed as follows:

$$
\begin{aligned}
\hat{\tau}_{f i}(t) & =\dot{f}(t) * M_{i} \dot{q}_{i}(t)+f(0) M_{i} \dot{q}_{i}(t)-f(t) M_{i} \dot{q}_{i}(0)+Y_{f i}\left(q_{i}(t), \dot{q}_{i}(t), \tau_{s i}(t)\right) \hat{\theta}_{i} \\
& +f(t) *\left\{\gamma_{i}^{-1} \tau_{s i}(t)+\Phi_{i}\left(\dot{q}_{i}(t)\right) \operatorname{sign}\left(\dot{q}_{i}(t)\right)\right\}
\end{aligned}
$$

and the parameters' estimate is generated using prediction error driven gradient update law that is designed using the switching $\sigma$-modification theory (Ioannou and Tsakalis 1986), to avoid parameter drift due to the modeling errors as follows:

$$
\dot{\hat{\theta}}_{i}(t)=\Gamma Y_{f i}^{T} \mathbf{e}_{i}-\Gamma \sigma_{s} \hat{\theta}_{i}
$$




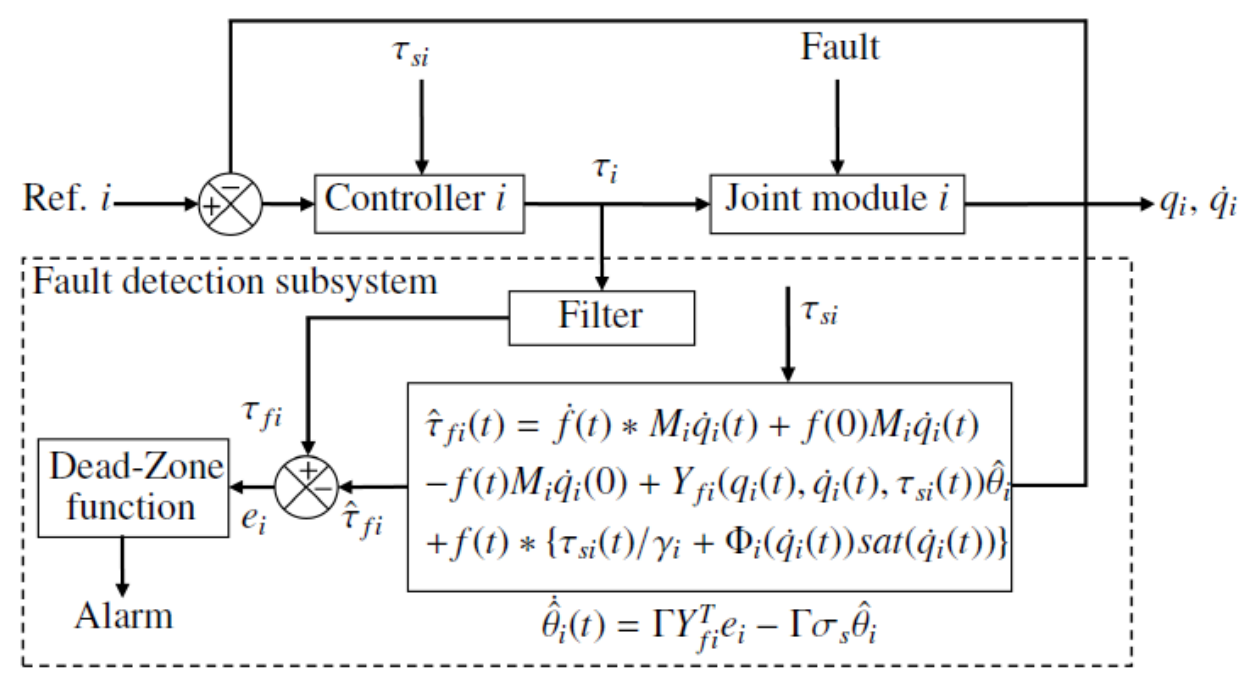

Figure 5.1: Block diagram of distributed fault detection of one MRR joint module.

where $\Gamma \in \Re^{4 \times 4}$ is adaptive gain with $\Gamma=\Gamma^{T}>0$ and $\sigma_{s} \in \Re$ is given by:

$$
\sigma_{s}=\left\{\begin{array}{lcc}
0 & \text { If } & \left|\hat{\theta}_{i}(t)\right|<M_{0} \\
\sigma_{0}\left(\frac{\left|\hat{\theta}_{i}(t)\right|}{M_{0}}-1\right) & \text { If } & M_{0} \leq\left|\hat{\theta}_{i}(t)\right| \leq 2 M_{0} \\
\sigma_{0} & \text { If } & \left|\hat{\theta}_{i}(t)\right| \geq 2 M_{0}
\end{array}\right.
$$

where $M_{0}>0, \sigma_{0}>0$ are design constants and $M_{0}$ is chosen to be large enough so that $M_{0}>\left|\theta_{i}\right|$. The prediction error in (5.14) becomes:

$$
\mathbf{e}_{i}(t)=-Y_{f i}\left(q_{i}(t), \dot{q}_{i}(t), \tau_{s i}(t)\right) \tilde{\theta}_{i}+\delta_{f i}(t)+\zeta_{f i}(t)
$$

A block diagram of the proposed fault detection scheme is shown in Fig. 5.1.

Theorem 2 The adaptive law represented by (5.22) and (5.23) guarantees that

i) $\hat{\theta}_{i}, \boldsymbol{e}_{i}, \dot{\boldsymbol{e}}_{i} \in \mathcal{L}_{\infty}$

ii) $\boldsymbol{e}_{i}, \dot{\hat{\theta}}_{i} \in \mathcal{S}\left(\bar{\delta}_{f i}+\sigma_{0}\right)$

where $\bar{\delta}_{f i}$ is the upper bound for the coupling term $\delta_{f i}$, i.e., $\bar{\delta}_{f i}=\sup _{t \geq 0} \delta_{f i}$.

iii) In the absence of modeling errors, i.e., when $\delta_{f i}=0$, property (ii) can be replaced with ii') $\boldsymbol{e}_{i}, \dot{\boldsymbol{e}}_{i}, \dot{\hat{\theta}}_{i} \in \mathcal{L}_{2}$

Therefore, from Barbălate's Lemma we have $\boldsymbol{e}_{i}(t) \rightarrow 0$ as $t \rightarrow \infty$, which is implied by $\boldsymbol{e}_{i}, \dot{e}_{i} \in \mathcal{L}_{\infty}, \boldsymbol{e}_{i} \in \mathcal{L}_{2}$. 
In order to facilitate the subsequent analysis, the following Lyapunov-like function $V\left(\tilde{\theta}_{i}(t)\right) \in \Re$ is defined as,

$$
V=\frac{\tilde{\theta}_{i}^{T} \Gamma^{-1} \tilde{\theta}_{i}}{2}
$$

the time derivative of which is given by the equation:

$$
\dot{V}=\tilde{\theta}_{i}^{T} \Gamma^{-1} \dot{\tilde{\theta}}_{i}
$$

Utilizing the fact that $\dot{\tilde{\theta}}_{i}(t)=\dot{\hat{\theta}}_{i}(t)$ and then substituting for 5.22 , one obtains:

$$
\dot{V}=Y_{f i} \tilde{\theta}_{i} \mathbf{e}_{i}-\sigma_{s} \tilde{\theta}_{i}^{T} \hat{\theta}_{i}
$$

From (5.24), one can get:

$$
Y_{f i} \tilde{\theta}_{i}=-\mathbf{e}_{i}+\delta_{f i}+\zeta_{f i}
$$

By substituting (5.28) into 5.27), one can obtain the following expression:

$$
\dot{V}=-e_{i}^{2}+\delta_{f i} \mathbf{e}_{i}-\sigma_{s} \tilde{\theta}_{i}^{T} \hat{\theta}_{i}+\mathbf{e}_{i} \zeta_{f i}
$$

In the absence of fault, $\zeta_{f i}=0$, therefore,

$$
\dot{V}=-e_{i}^{2}+\delta_{f i} \mathbf{e}_{i}-\sigma_{s} \tilde{\theta}_{i}^{T} \hat{\theta}_{i} \leq-\mathbf{e}_{i}^{2}+\left|\mathbf{e}_{i}\right| \bar{\delta}_{f i}-\sigma_{s} \tilde{\theta}_{i}^{T} \hat{\theta}_{i}
$$

where $\bar{\delta}_{f i}$ is the upper bound for the coupling term $\delta_{f i}$, i.e., $\bar{\delta}_{f i}=\sup _{t \geq 0} \delta_{f i}$. Using completion of squares, one can write:

$$
-\mathbf{e}_{i}^{2}+\left|\mathbf{e}_{i}\right| \bar{\delta}_{f i} \leq-\frac{\mathbf{e}_{i}^{2}}{2}-\frac{1}{2}\left[\mathbf{e}_{i}-\bar{\delta}_{f i}\right]^{2}+\frac{\bar{\delta}_{f i}^{2}}{2} \leq-\frac{\mathbf{e}_{i}^{2}}{2}+\frac{\bar{\delta}_{f i}^{2}}{2}
$$

and,

$$
\sigma_{s} \tilde{\theta}_{i}^{T} \hat{\theta}_{i}=\sigma_{s}\left(\left|\hat{\theta}_{i}\right|^{2}-\theta_{i}^{T} \hat{\theta}_{i}\right) \geq \sigma_{s}\left|\hat{\theta}_{i}\right|\left(\left|\hat{\theta}_{i}\right|-M_{0}+M_{0}-\left|\theta_{i}\right|\right)
$$

Because $\sigma_{s} \geq 0, \sigma_{s}\left(\left|\hat{\theta}_{i}\right|-M_{0}\right) \geq 0$ and $M_{0}>\left|\theta_{i}\right|$, it follows that:

$$
\sigma_{s} \tilde{\theta}_{i}^{T} \hat{\theta}_{i} \geq \sigma_{s}\left|\hat{\theta}_{i}\right|\left(\left|\hat{\theta}_{i}\right|-M_{0}\right)+\sigma_{s}\left|\hat{\theta}_{i}\right|\left(M_{0}-\left|\theta_{i}\right|\right) \geq \sigma_{s}\left|\hat{\theta}_{i}\right|\left(M_{0}-\left|\theta_{i}\right|\right) \geq 0
$$

From (5.33), it can be concluded $-\sigma_{s} \tilde{\theta}_{i}^{T} \hat{\theta}_{i} \leq 0$, which implies that this term would only make $\dot{V}$ more negative. Because for $\left|\hat{\theta}_{i}\right|=\left|\tilde{\theta}_{i}+\theta_{i}\right|>2 M_{0}$, the term $-\sigma_{s} \tilde{\theta}_{i}^{T} \hat{\theta}_{i}=-\sigma_{0} \tilde{\theta}_{i}^{T} \hat{\theta}_{i}$. We also have,

$$
-\sigma_{0} \tilde{\theta}_{i}^{T} \hat{\theta}_{i}=-\sigma_{0} \tilde{\theta}_{i}^{T}\left(\tilde{\theta}_{i}+\theta_{i}\right) \leq-\sigma_{0}\left(\left|\tilde{\theta}_{i}\right|^{2}+\left|\tilde{\theta}_{i}\right|\left|\theta_{i}\right|\right)
$$


Using the inequality $-a^{2} \pm a b \leq-\frac{a^{2}}{2}+\frac{b^{2}}{2}$, one obtains:

$$
-\sigma_{0}\left(\left|\tilde{\theta}_{i}\right|^{2}+\left|\tilde{\theta}_{i}\right|\left|\theta_{i}\right|\right) \leq-\sigma_{0} \frac{\left|\tilde{\theta}_{i}\right|^{2}}{2}+\sigma_{0} \frac{\left|\theta_{i}\right|^{2}}{2}
$$

Therefore, from (5.31) and 5.35) one can rewrite (5.30) as:

$$
\dot{V} \leq-\frac{\mathbf{e}_{i}^{2}}{2}-\sigma_{0} \frac{\left|\tilde{\theta}_{i}\right|^{2}}{2}+\frac{\bar{\delta}_{f i}^{2}}{2}+\sigma_{0} \frac{\left|\theta_{i}\right|^{2}}{2}
$$

Adding and subtracting the term $\alpha V$ for some $\alpha>0$, one obtains:

$$
\dot{V} \leq-\alpha V+\alpha\left(\frac{\tilde{\theta}_{i}^{T} \Gamma^{-1} \tilde{\theta}_{i}}{2}\right)-\frac{\mathbf{e}_{i}^{2}}{2}-\sigma_{0} \frac{\left|\tilde{\theta}_{i}\right|^{2}}{2}+\frac{\bar{\delta}_{f i}^{2}}{2}+\sigma_{0} \frac{\left|\theta_{i}\right|^{2}}{2}
$$

Because $\tilde{\theta}_{i}^{T} \Gamma^{-1} \tilde{\theta}_{i} \leq\left|\tilde{\theta}_{i}\right|^{2} \lambda_{\max }\left(\Gamma^{-1}\right)$, equation (5.37) can be rewritten as:

$$
\begin{gathered}
\dot{V} \leq-\alpha V+\alpha\left(\frac{\left|\tilde{\theta}_{i}\right|^{2} \lambda_{\max }\left(\Gamma^{-1}\right)}{2}\right)-\frac{\mathbf{e}_{i}^{2}}{2}-\sigma_{0} \frac{\left|\tilde{\theta}_{i}\right|^{2}}{2}+\frac{\bar{\delta}_{f i}^{2}}{2}+\sigma_{0} \frac{\left|\theta_{i}\right|^{2}}{2} \\
\dot{V} \leq-\alpha V-\left(\sigma_{0}-\alpha \lambda_{\max }\left(\Gamma^{-1}\right)\right) \frac{\left|\tilde{\theta}_{i}\right|^{2}}{2}-\frac{\mathbf{e}_{i}^{2}}{2}+\frac{\bar{\delta}_{f i}^{2}}{2}+\sigma_{0} \frac{\left|\theta_{i}\right|^{2}}{2}
\end{gathered}
$$

If we choose $0<\alpha \leq \frac{\sigma_{0}}{\lambda_{\max }\left(\Gamma^{-1}\right)}$,

$$
\dot{V} \leq-\alpha V+\frac{\bar{\delta}_{f i}^{2}}{2}+\sigma_{0} \frac{\left|\theta_{i}\right|^{2}}{2}
$$

Hence, for $V \geq V_{0}=\frac{1}{2 \alpha}\left(\bar{\delta}_{f i}^{2}+\sigma_{0}\left|\theta_{i}\right|^{2}\right), \quad \dot{V} \leq 0$, which implies that $V \in \mathcal{L}_{\infty}$ and therefore $\tilde{\theta}_{i}, \hat{\theta}_{i} \in \mathcal{L}_{\infty}$. Furthermore, according to 5.6 and Assumption 1, $Y_{f i}, \dot{Y}_{f i}, \tau_{f i}, \dot{\tau}_{f i} \in \mathcal{L}_{\infty}$. Based on the fact that $Y_{f i}, \tilde{\theta}_{i} \in \mathcal{L}_{\infty}$, one can utilize (5.24) to conclude that $\mathbf{e}_{i} \in \mathcal{L}_{\infty}$. Since $Y_{f i}, \mathbf{e}_{i} \in \mathcal{L}_{\infty}$ it can be concluded from 5.22 that $\hat{\theta}_{i} \in \mathcal{L}_{\infty}$. We can also use $-\sigma_{0} \tilde{\theta}_{i}^{T} \hat{\theta}_{i} \leq$ $-\sigma_{0} \frac{\left|\hat{\theta}_{i}\right|^{2}}{2}+\sigma_{0} \frac{\left|\theta_{i}\right|^{2}}{2}$ and rewrite 5.30 as:

$$
\dot{V} \leq-\frac{\mathbf{e}_{i}^{2}}{2}+\frac{\bar{\delta}_{f i}}{2}-\sigma_{0} \frac{\left|\hat{\theta}_{i}\right|^{2}}{2}+\sigma_{0} \frac{\left|\theta_{i}\right|^{2}}{2}
$$

Integrating both sides of (5.41) yields:

$$
\begin{aligned}
\int_{t}^{t+T}\left(\mathbf{e}_{i}^{2}+\sigma_{0}\left|\hat{\theta}_{i}\right|^{2}\right) d \tau & \leq \int_{t}^{t+T}\left(\bar{\delta}_{f i}+\sigma_{0}\left|\theta_{i}\right|^{2}\right) d \tau+2[V(t)-V(t+T)] \\
& \leq c_{0}\left(\bar{\delta}_{f i}+\sigma_{0}\right)+c_{1}
\end{aligned}
$$


$\forall t \geq 0$ and $T>0$ where $c_{0}=\max \left[1,\left|\theta_{i}\right|^{2}\right], c_{1}=2 \sup _{t}[V(t)-V(t+T)]$, which implies that $\mathbf{e}_{i}, \sqrt{\sigma_{0}} \hat{\theta} \in \mathcal{S}\left(\bar{\delta}_{f i}+\sigma_{0}\right)$. In addition, from equation (5.22), it follows that:

$$
\dot{\hat{\theta}}_{i} \in \mathcal{S}\left(\bar{\delta}_{f i}+\sigma_{0}\right)
$$

Furthermore, when the coupling term $\delta_{f i}=0$, because $-\sigma_{s} \tilde{\theta}_{i}^{T} \hat{\theta}_{i} \leq 0$ equation 5.30 implies that $\mathbf{e}_{i} \in \mathcal{L}_{2}$. Based on the facts that $Y_{f i}, \dot{Y}_{f i}, \mathbf{e}_{i}, \dot{\hat{\theta}}_{i} \in \mathcal{L}_{\infty}$, it can be concluded that $\dot{e}_{i} \in \mathcal{L}_{\infty}$. Therefore, since $\mathbf{e}_{i}, \dot{e}_{i} \in \mathcal{L}_{\infty}$ and $\mathbf{e}_{i} \in \mathcal{L}_{2}$, Barbălate's Lemma can be utilized to conclude that $\lim _{t \rightarrow \infty}\left|\mathbf{e}_{i}\right|=0$ in the absence of fault. Therefore, the boundedness of the prediction error $\mathbf{e}_{i}$ is proved.

The residual given in 5.20 can be used to determine if an actuator fault is present in any MRR joint module. The fact that the residual given in 5.20 is based on fixed conservative threshold, motivates one to design a new dead-zone update rule to modify the dead-zone threshold as $\left|\mathbf{e}_{i}\right|$ decreases. The prediction error converges to a small value as the filtered torque estimate $\hat{\tau}_{f i}$ converges to a value close to the filtered torque $\tau_{f i}$. The upper bound of the prediction error, $\varrho c_{i}$, and the convergence time, $t_{c i}$, are determined experimentally for each joint module in the absence of a fault. Once $\varrho c_{i}$ and $t c_{i}$ are known, the following dead-zone residual function is defined to indicate a fault:

$$
D\left(\mathbf{e}_{i}\right)=\left\{\begin{array}{ccc}
1 & \text { if } & \left|\mathbf{e}_{i}\right|>\varrho_{i}(t) \\
0 & \text { if } & \left|\mathbf{e}_{i}\right| \leq \varrho_{i}(t)
\end{array}\right.
$$

where $\varrho_{i}(t)$ is selected as follows:

$$
\begin{aligned}
& \text { if } \quad\left(t \leq t c_{i}\right) \quad \text { then } \varrho_{i}(t)=\left|\mathbf{e}_{i}\right|+\epsilon \\
& \text { else } \varrho_{i}(t)=\varrho c_{i}
\end{aligned}
$$

and $\epsilon$ is a small positive constant.

Remark 7 A similar dead-zone update rule can be designed using sliding window technique, the dimensions of which are user-defined through experimental observations. Upon the settlement of prediction error within the tolerances of sliding window, the upper bound of the prediction error can be measured and utilized as threshold, $\varrho c_{i}$. A point to be noted is that guaranteeing of $\varrho c_{i}$ as the true upper bound of $\boldsymbol{e}_{i}$ in the absence of a fault is not really possible. Therefore, there is always a possibility of false alarms. Since $\boldsymbol{e}_{i} \in \mathcal{L}_{2}$, the probability of false alarms 
Table 5.1: Physical parameters of the two-link MRR robot

\begin{tabular}{|c|c|c|}
\hline Parameter & Joint 1 & Joint 2 \\
\hline \multicolumn{2}{|c|}{ Datasheet-based parameters } \\
\hline Rotor inertia (kg-m2) & $0.168 \times 10^{-4}$ & $0.168 \times 10^{-4}$ \\
\hline Gear ratio & 101 & 101 \\
\hline \multicolumn{2}{|c|}{ Experimentally identified friction parameters } \\
\hline Viscous friction coefficient (Nms/rad) & 0.12 & 0.1 \\
\hline Static friction parameter (Nm) & 0.0152 & 0.0143 \\
\hline Coulomb friction parameter (Nm) & 0.015 & 0.0124 \\
\hline Stribeck effect parameter $(\mathrm{Nm})$ & 100 & 90 \\
\hline$g_{1}$ & 4.9 & 5.2 \\
\hline$g_{2}$ & 0.6 & 0.8 \\
\hline
\end{tabular}

can be practically eliminated by choosing appropriately long lengths of the sliding window. This dead-zone update rule has the advantage that it eliminates the requirement of convergence time, $t_{c i}$.

\subsection{Experimental Results}

The proposed distributed fault detection scheme was implemented on two-degrees-of-freedom revolute MRR robot as shown in Fig. 5.2. The dynamics of this two-link MRR robot are described in equations (3.1) and (3.2). The rotor inertia and harmonic drive's reduction ratio of each joint are provided by the manufacturer and the friction model parameters have been experimentally determined. The dynamic model parameters are given in Table 5.1 with all parameters measured in SI units in the joint space. Our new generation MRR joint module with joint toque sensing, joint 1 in Fig. 5.2, is used as the first and second joint by simply switching its position. The harmonic drive of this MRR joint is driven by a brushed DC motor from Maxon which is controlled through torque controlled Quanser linear power amplifier. The data acquisition, control implementation, and fault detection were performed using the Quanser Q8 data acquisition card at a frequency of $1 \mathrm{kHz}$. Distributed control method based on joint torque sensing (Ahmad et al. 2013) is used to ensure desired position tracking of each joint 


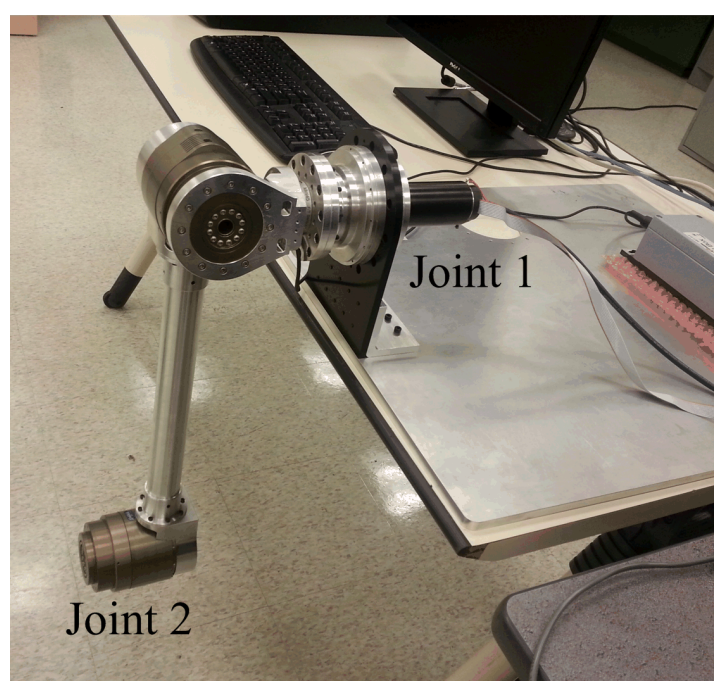

Figure 5.2: Two-link MRR robot.

with a reasonable performance. The desired trajectory for both joints is represented by the following sigmoid function:

$$
q_{d i}(t)=0.5 A m p\left(1-\cos \left(\pi n \frac{t}{t_{e x}}\right)\right) \text { for } 0 \leq t \leq 30 s
$$

where, $A m p=10 \mathrm{deg}$ is the sigmoid amplitude, $t_{e x}=30 \mathrm{~s}$ is the experiment runtime, and $n=12$ is a constant used to control the number of sigmoids. Several experiments were conducted to demonstrate the effectiveness of the proposed scheme. In the first experiment, the nominal values of friction model parameters of joint 1 are used to calculate the filtered torque estimates in (5.15). The results of this experiment are depicted in Fig. 5.3. The filtered torque and filtered torque estimate are plotted in Fig. 5.3(a), the prediction error in Fig. 5.3(b), while the torque sensor measurements are shown in Fig. 5.3(c). This experiment was used to validate the proposed fault detection scheme under relatively small parameters uncertainties and modeling errors. External torque was applied to the first link in both directions to show that the proposed fault detection scheme achieves good performance in the presence of external disturbance, i.e., contact with objects in surrounding or unknown payload. This is a key issue in most previously proposed fault detection schemes for robot manipulators. The reasons of it being the facts that: i) most fault detections schemes are based on dynamic model of the system having unknown external torque if not measured or estimated, and ii) the joint friction highly depends on the load and therefore, false alarms are expected to occur in the presence of any external torque. A major drawback that hinders the reliability of model-based FDI schemes is the model uncertainty, which could be caused due to reasons like parameter variations and/or linearization 
errors, for example. The friction model parameters may change over time which makes false alarms inevitable if the nominal values of the friction parameters are used to calculate the filtered torque estimates. A compromise between the false alarms rates and sensitivity to small faults can be achieved by adjusting the threshold used in the dead-zone function. The decision making can be improved by using methods of change detection, e.g., a fuzzy or adaptive threshold, Bayes decision, or likelihood-ration test.

In the proposed distributed fault detection scheme, the friction model parameters are estimated online to minimize the effect of parameters uncertainty. Additionally, a friction model that incorporates payload dependency was utilized to achieve small prediction error that is independent of the payload variation. For the experimental verification of the distributed fault detection scheme, the friction parameters are assumed to be unknown or have changed over time due to lubricating and/or aging condition. The initial estimate of friction parameters, $\hat{\theta}_{i}(0)$, were selected to be $\pm 30 \%$ of their actual values. The parameters estimate, $\hat{\theta}_{i}(t)$, of equation (5.21) was generated online using (5.22). The gains and parameters of the adaptive update law in (5.22) were selected as follows: $\sigma_{0}=0.1, M_{0}$ was chosen using the experimentally determined values of the friction parameters $\theta_{i}$, and the filter parameters are $\beta=10$ and $\alpha=1$ for both joints.

$$
\Gamma=\operatorname{Diag}\{3.5,2,2.25,3\}
$$

Remark 8 The adaptive law (5.22) requires the knowledge of upper bound $M_{0}$ for $\left|\hat{\theta}_{i}(t)\right|$. Due to the fact that any unpermitted deviation of the system parameters from the acceptable, usual or standard condition is considered a fault, selection of value for $M_{0}$ requires a compromise between fault detection sensitivity and false alarms.

A free-swinging actuator fault, a ramp actuator fault with $\vartheta_{i}=1$, and a saturation actuator fault were injected into the two-link MRR system at $t=29 \mathrm{~s}$ for joint 1 and joint 2 in the second and third experiments, respectively. The results of the last two experiments are shown in Figs. 5.45 .7 for joint 1 and Figs. 5.85 .11 for joint 2. The topmost plots in Figs. 5.45 .11 depicts the filtered torques and filtered torque estimates, the middle ones depict the prediction error in full time scale, and the lowermost plots depicts the last $1.5 \mathrm{~s}$ of experimental runtime to highlight when the fault is detected. The lower plots in Fig. 5.4 and Fig. 5.8 are exception as they depict the torque sensor measurements to show the applied external torques. The interval between each instance of fault occurrence and fault detection is provided in Table 5.2 for each fault type. The fact that faults are detected while the tracking error is still smaller than the controller error can be easily noticed by observing the recorded delay times. It guarantees the stability of the MRR joint modules before or during fault detection. For example, most robot 


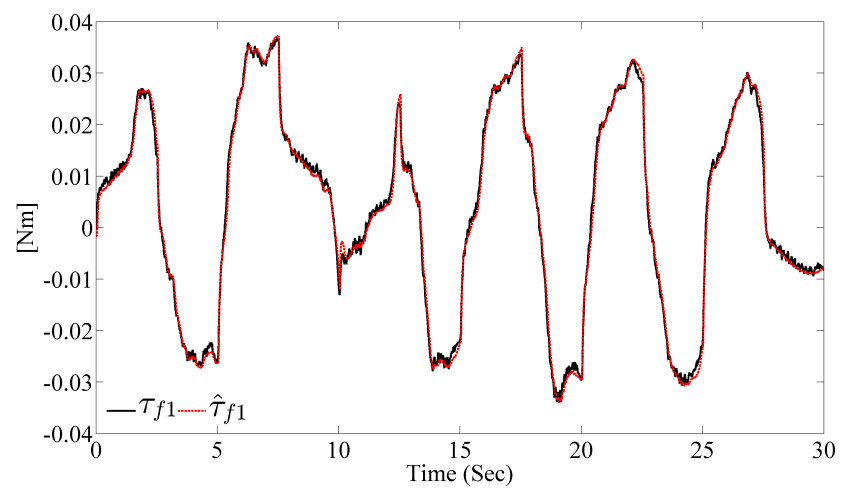

(a) Filtered torques

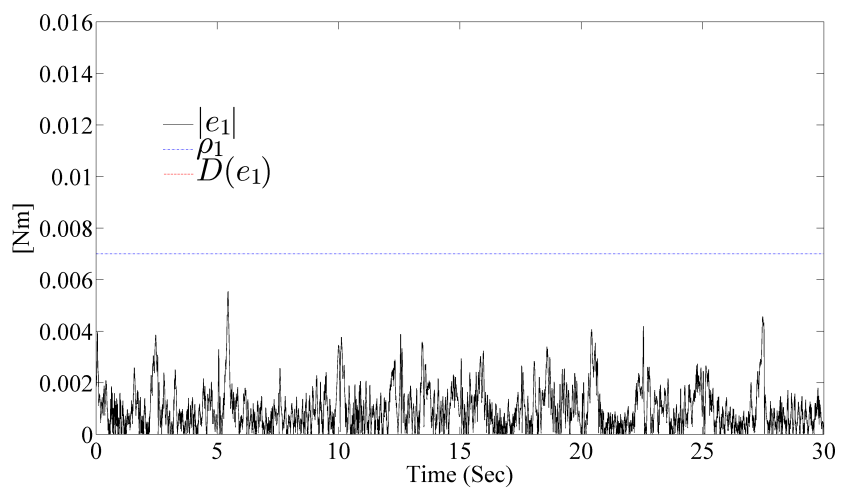

(b) Prediction error

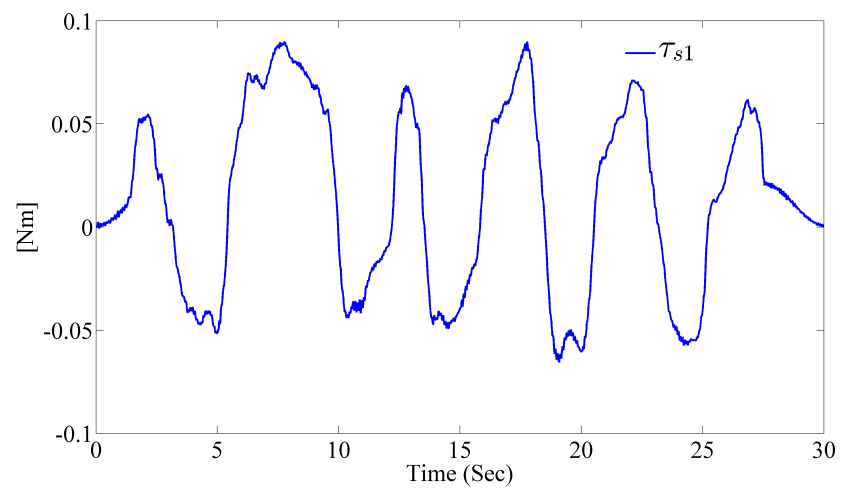

(c) Torque sensor readings

Figure 5.3: Fault free with nominal values, joint 1. 
manipulators work at joint velocities of around $2 \mathrm{rpm}$ or $12 \mathrm{deg} / \mathrm{sec}$ for which

$$
\dot{q}=\frac{\Delta q}{\Delta t}
$$

This implies that after $5 \mathrm{~ms}$ and at a joint velocity $\dot{q}=12 \mathrm{deg} / \mathrm{sec}$, the link-side error would only be $0.06 \mathrm{deg}$. At joint velocity of $5 \mathrm{deg} / \mathrm{sec}$, a $5 \mathrm{~ms}$ delay in the fault detection will only generate an insignificant $0.025 \mathrm{deg}$ error in the link-side.

Table 5.2: Delay times between the actuator faults occurrence and the fault detection.

\begin{tabular}{|c|c|c|}
\hline \multirow{2}{*}{ Fault Type } & \multicolumn{2}{|c|}{ Time delay (ms) } \\
\cline { 2 - 3 } & Joint 1 & Joint 2 \\
\hline \hline $\begin{array}{c}\text { Free-swinging actuator fault } \\
\text { Ramp actuator fault } \\
\text { Saturated actuator fault }\end{array}$ & 2 & 2 \\
\cline { 2 - 3 } Say & 3 & 2 \\
\hline
\end{tabular}




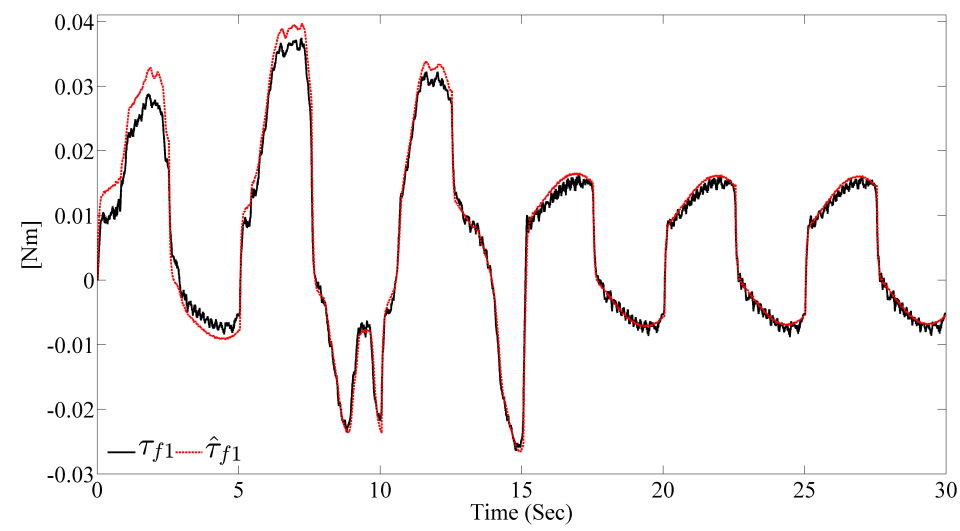

(a) Filtered torques

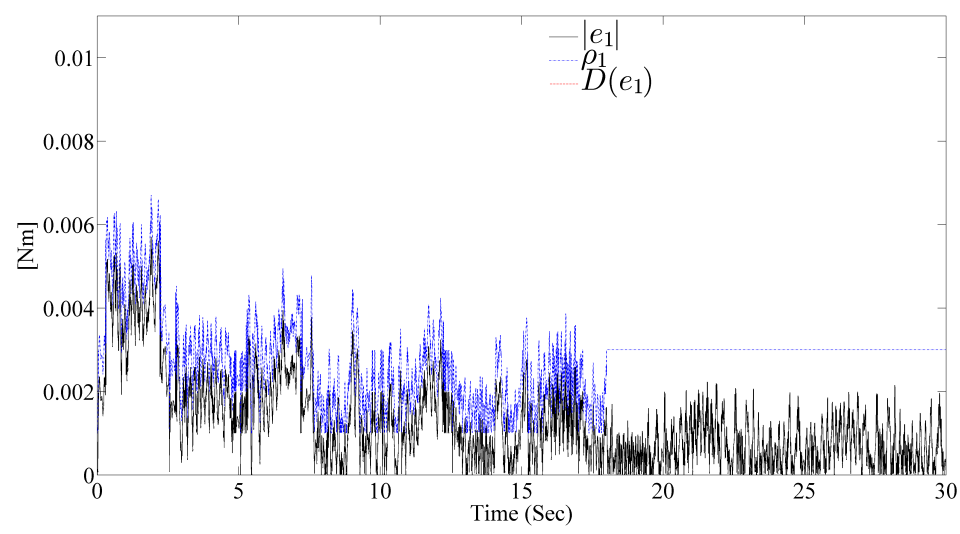

(b) Prediction error

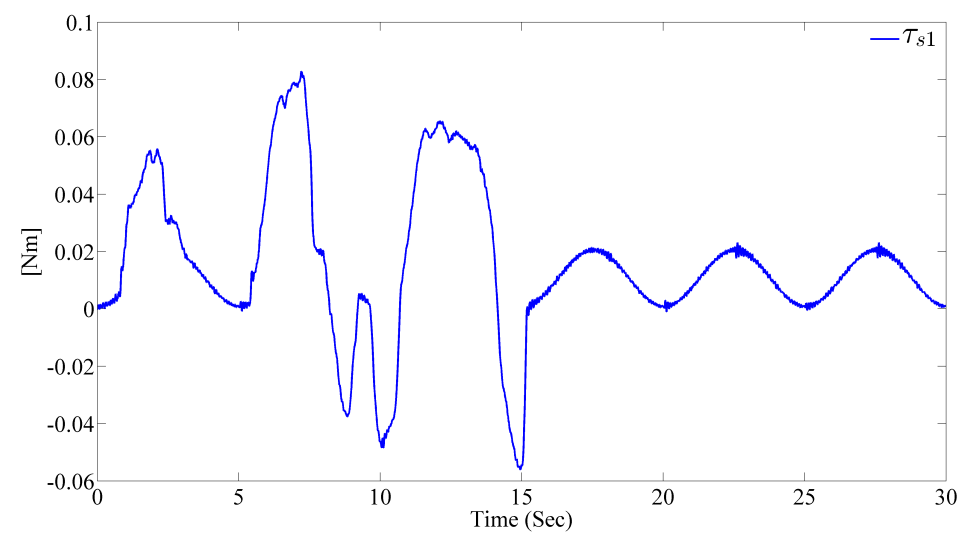

(c) Torque sensor readings

Figure 5.4: Fault free, joint 1. 


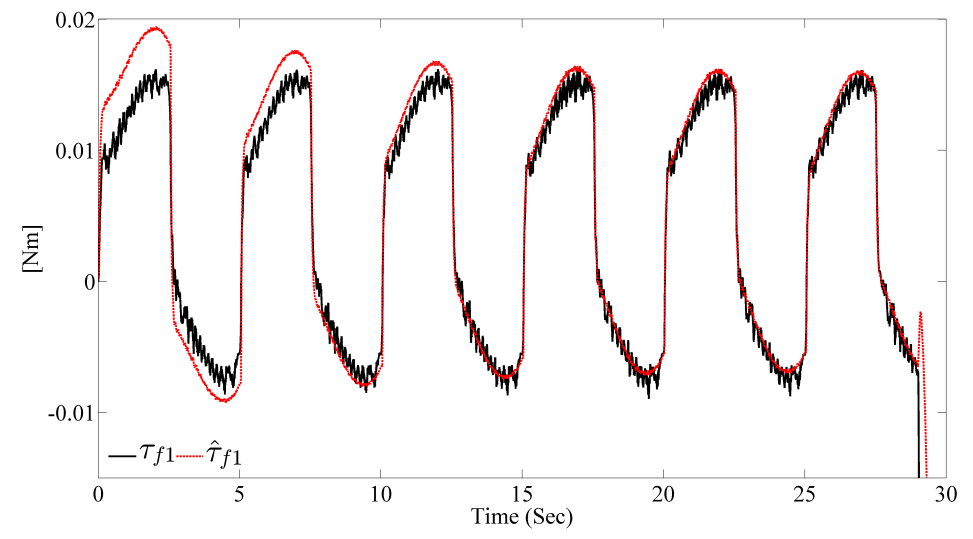

(a) Filtered torques

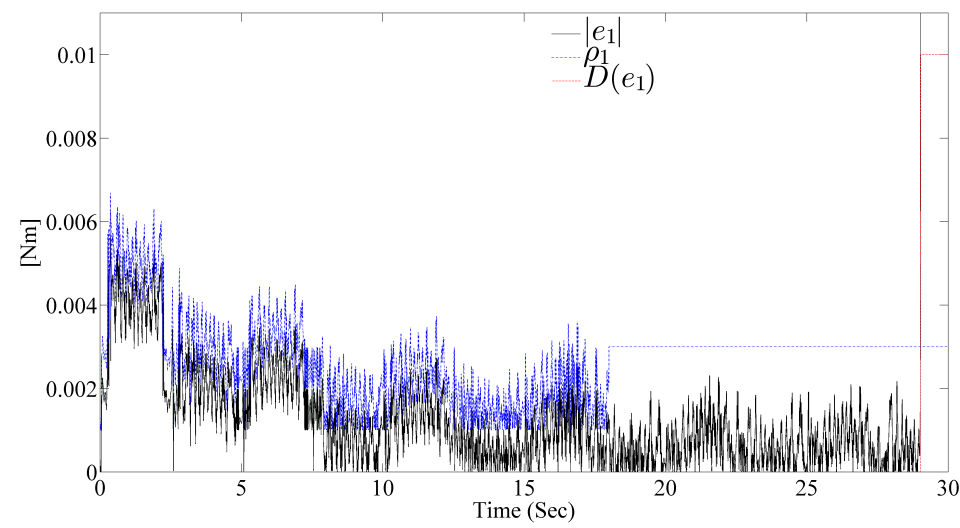

(b) Prediction error

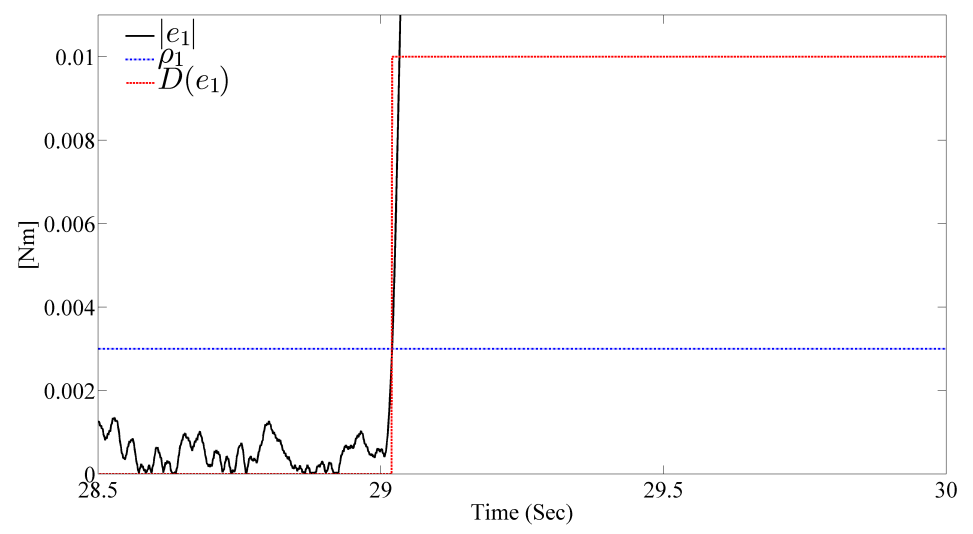

(c) Prediction error (zoom-in)

Figure 5.5: Free-swinging actuator fault, joint 1. 


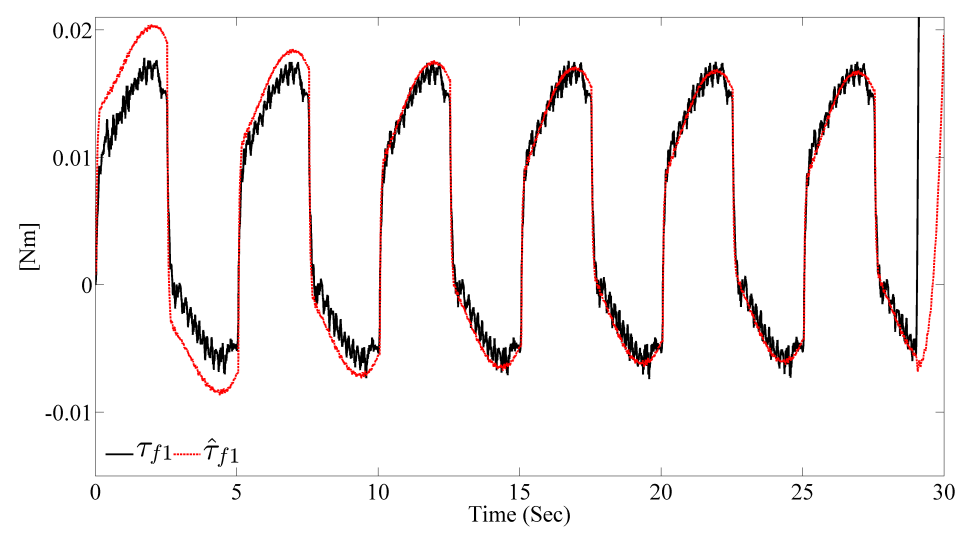

(a) Filtered torques

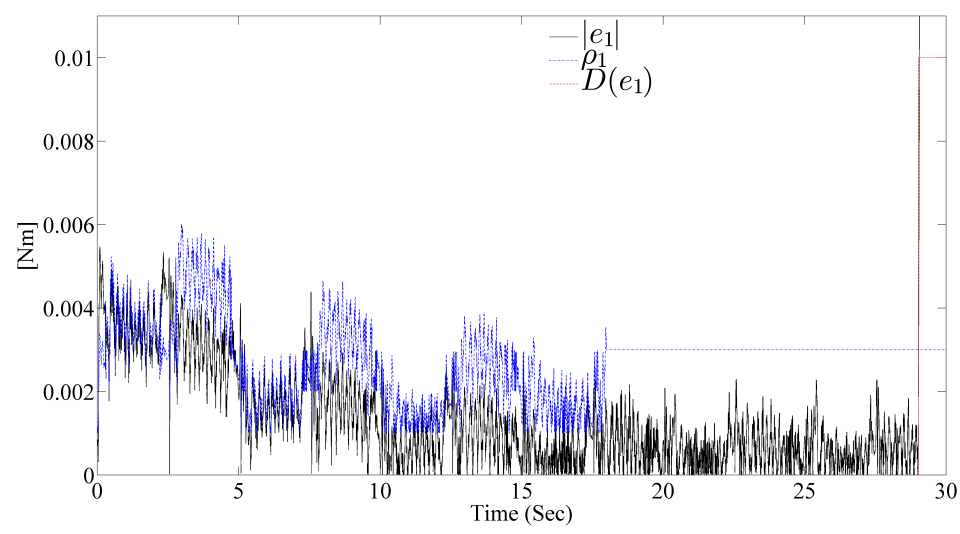

(b) Prediction error

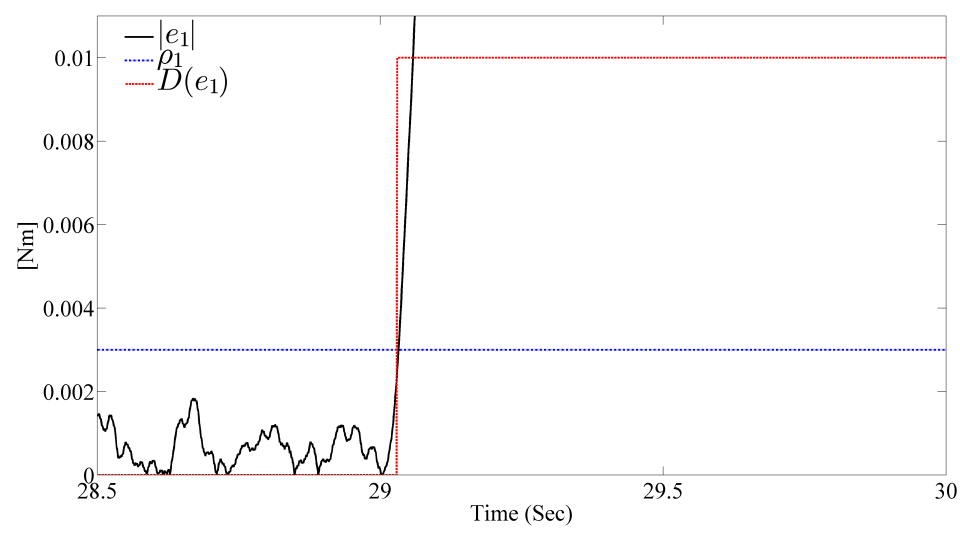

(c) Prediction error (zoom-in)

Figure 5.6: Ramp actuator fault, joint 1. 


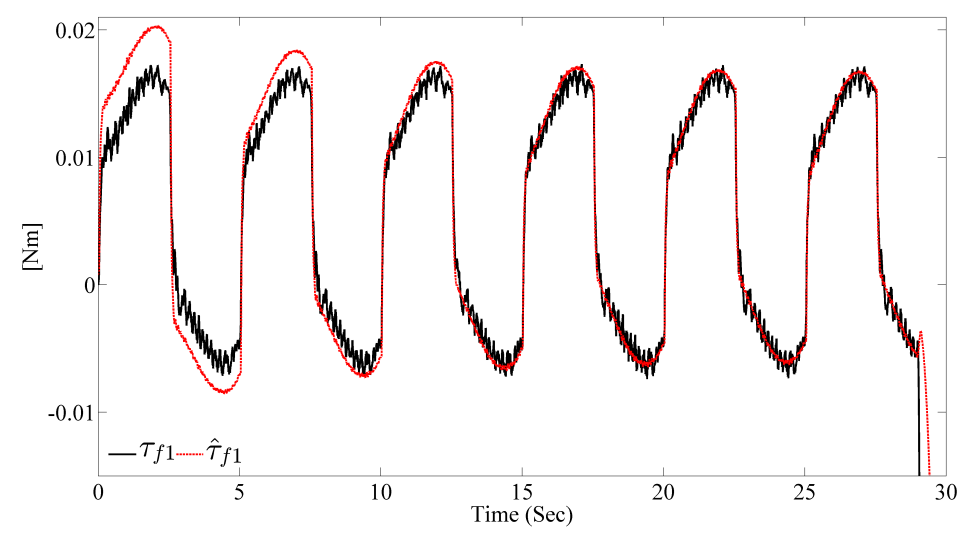

(a) Filtered torques

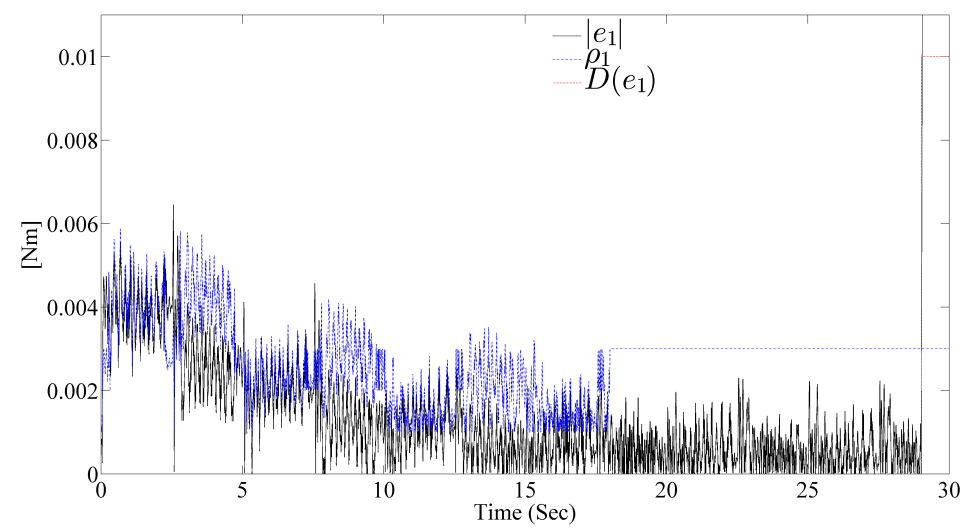

(b) Prediction error

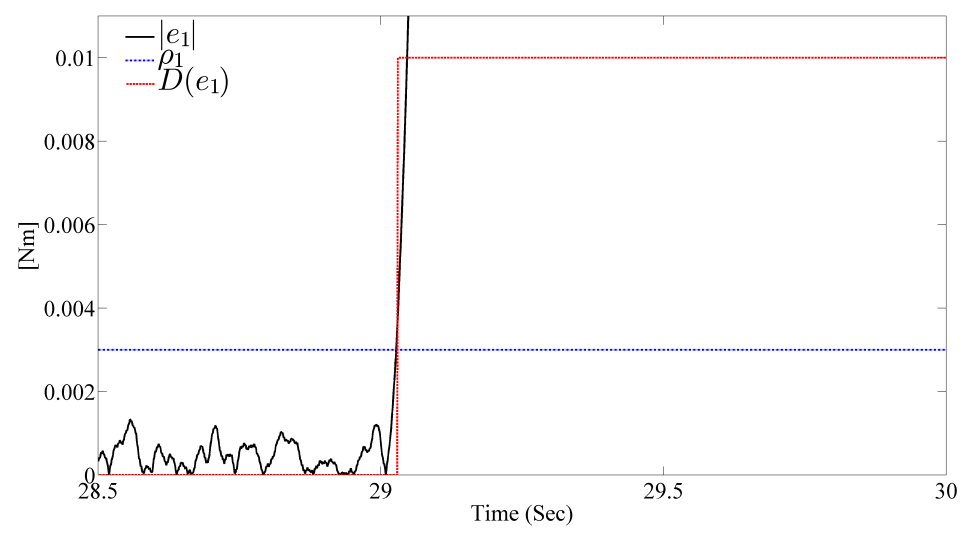

(c) Prediction error (zoom-in)

Figure 5.7: Saturated actuator fault, joint 1. 


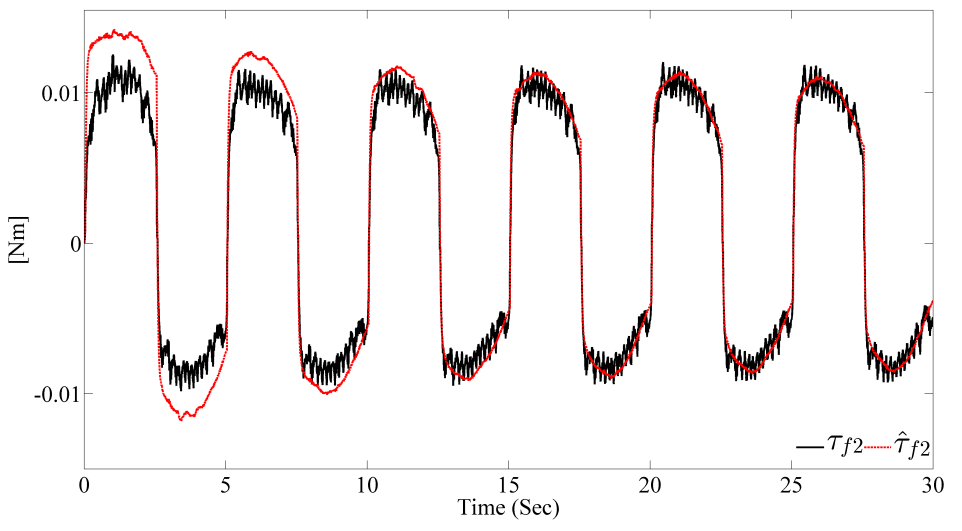

(a) Filtered torques

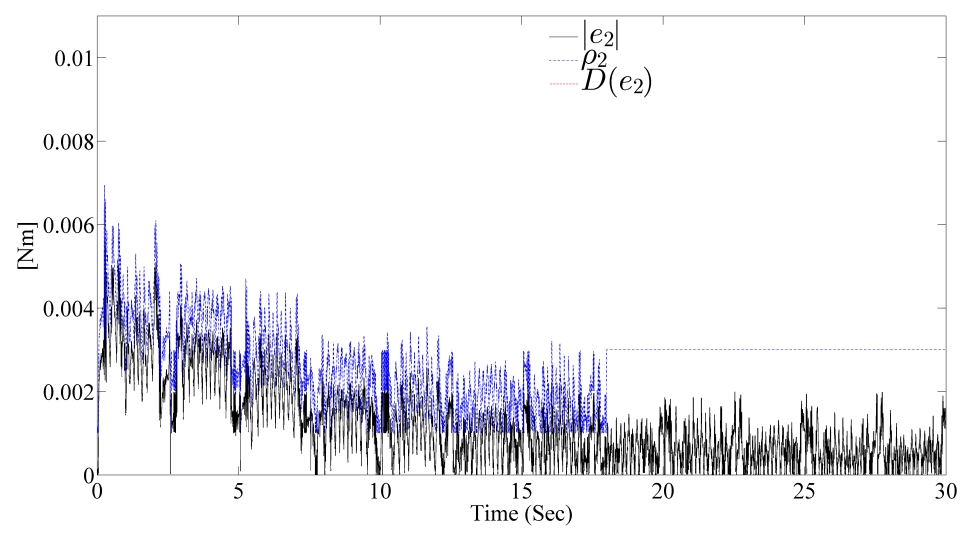

(b) Prediction error

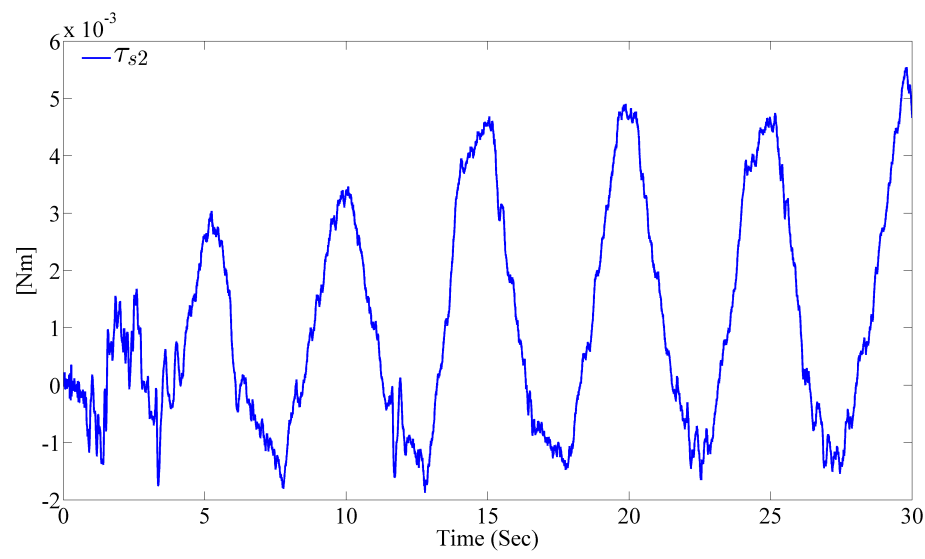

(c) Torque sensor readings

Figure 5.8: Fault free, joint 2. 


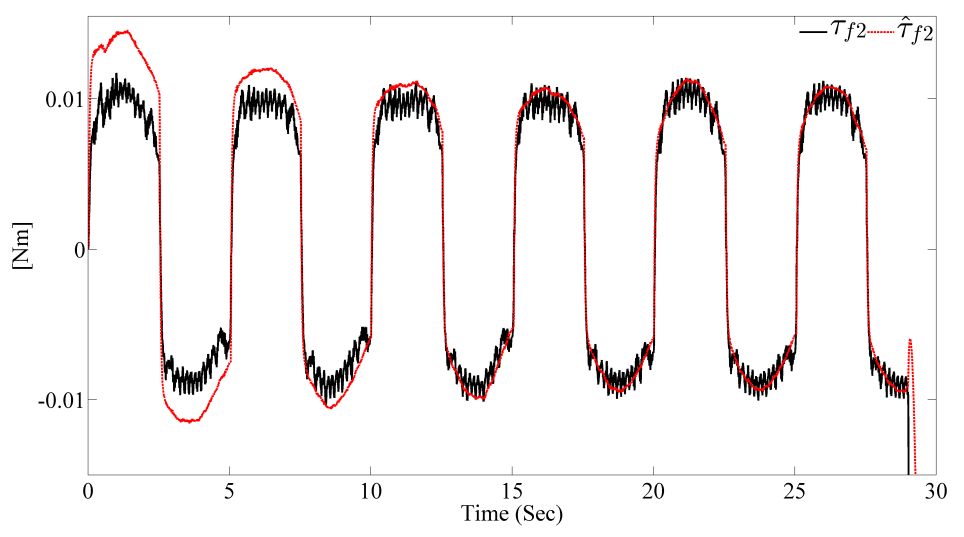

(a) Filtered torques

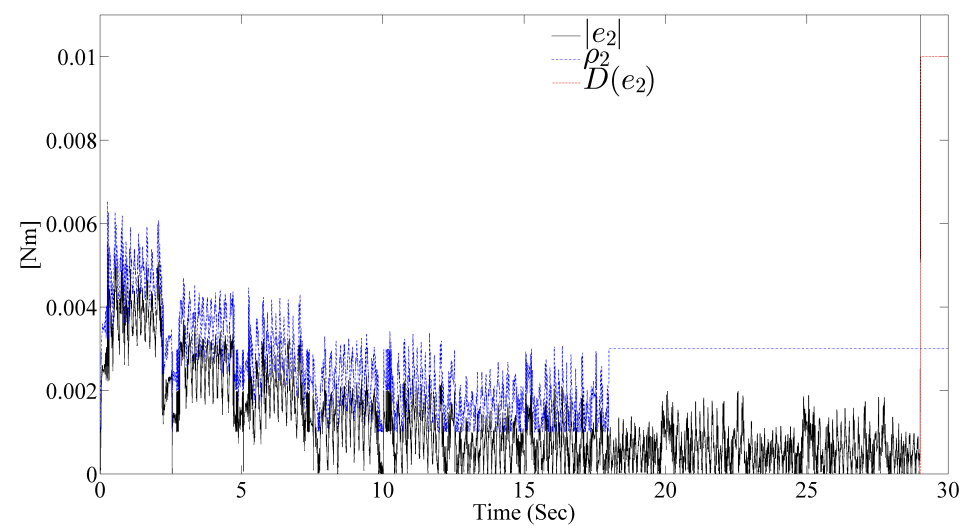

(b) Prediction error

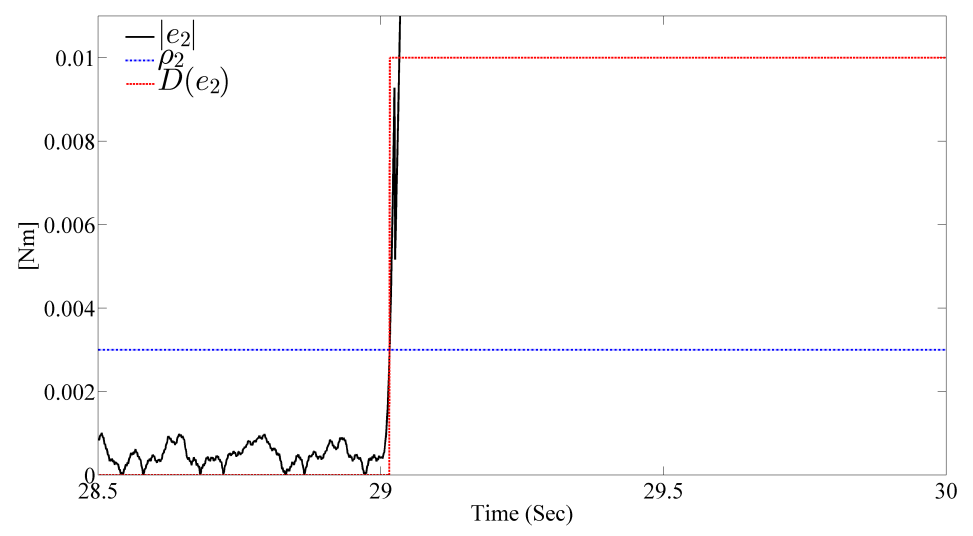

(c) Prediction error (zoom-in)

Figure 5.9: Free-swinging actuator fault, joint 2. 


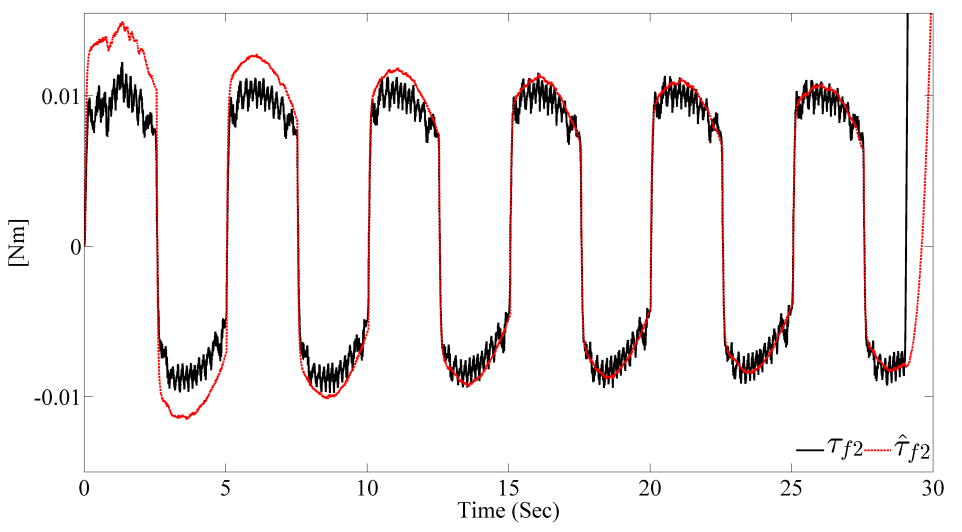

(a) Filtered torques

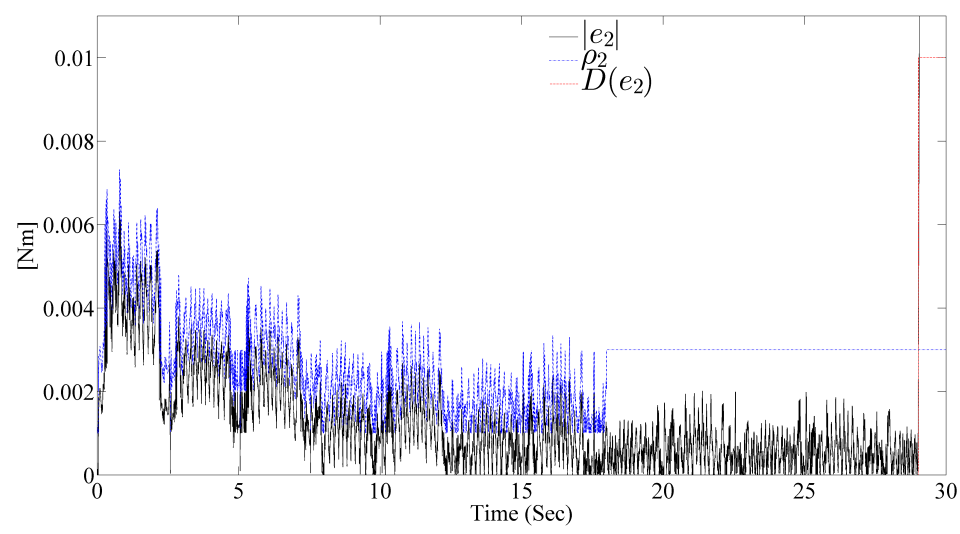

(b) Prediction error

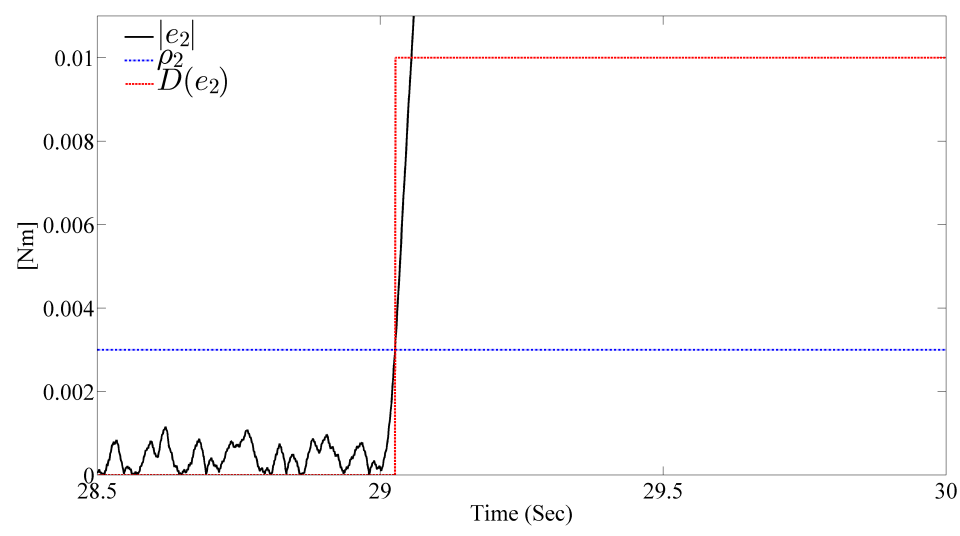

(c) Prediction error (zoom-in)

Figure 5.10: Ramp actuator fault, joint 2. 


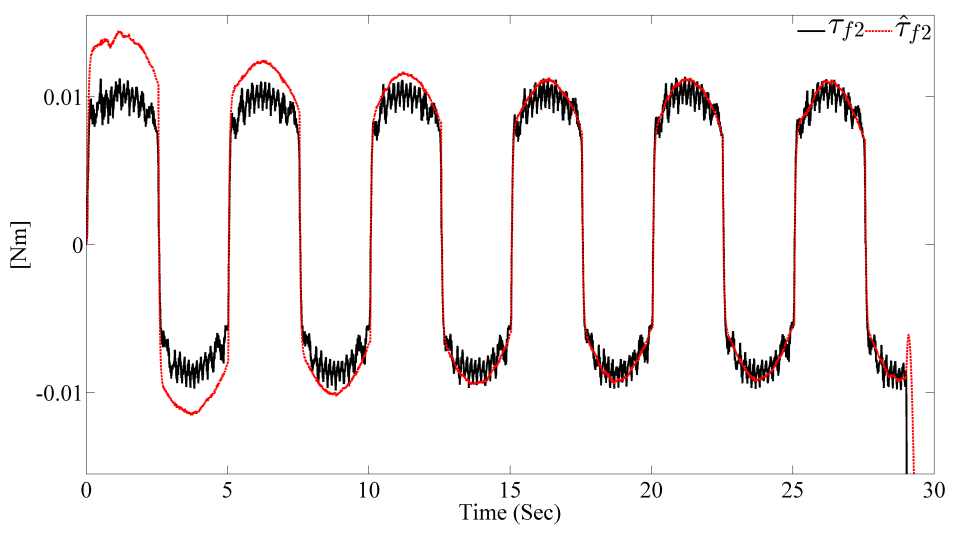

(a) Filtered torques

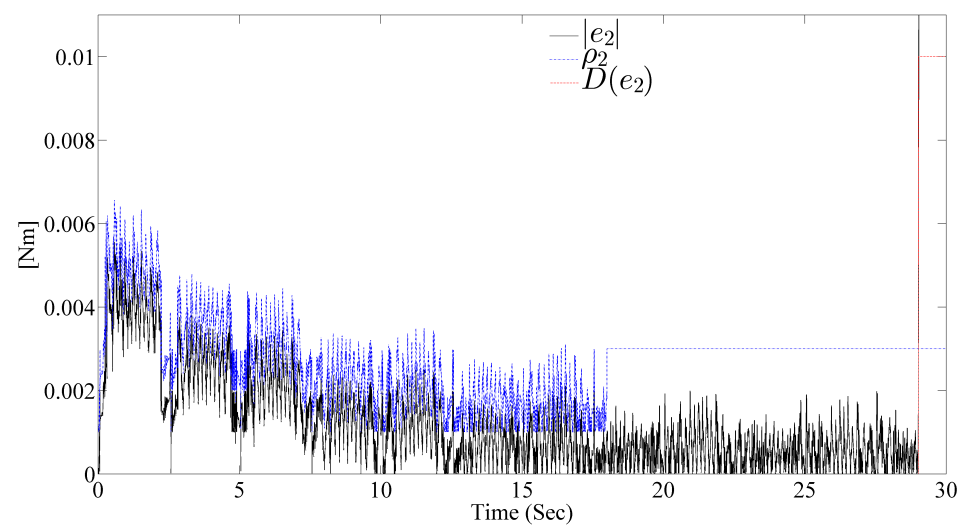

(b) Prediction error

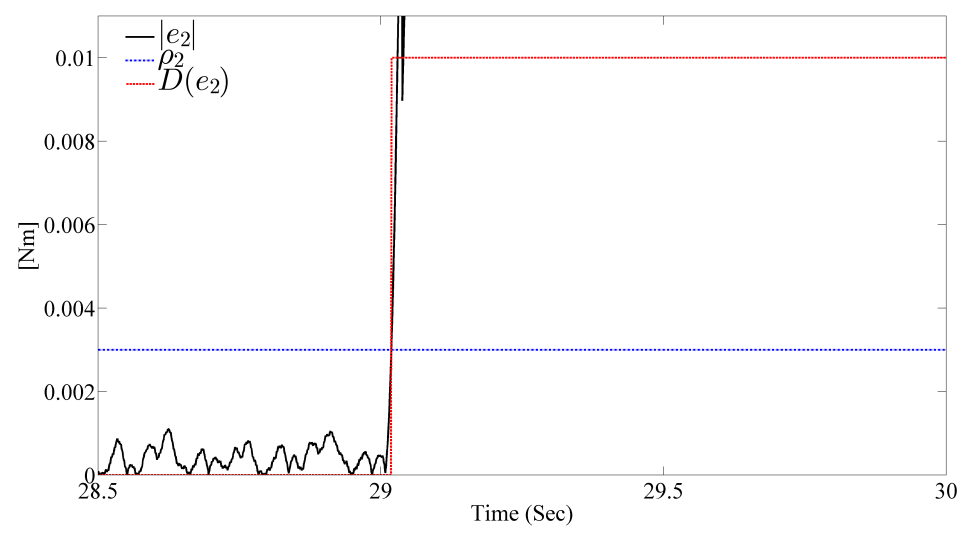

(c) Prediction error (zoom-in)

Figure 5.11: Saturated actuator fault, joint 2. 


\subsection{Concluding Remarks}

In the research work presented in this chapter, a distributed fault detection scheme that does not require motion states of any other modules is proposed for modular and reconfigurable robots with joint torque sensing. Unlike some previously proposed fault detection schemes, it's independent of measurements or estimations of joint accelerations. Since precise knowledge of the robot model and parameters may not be possible, the proposed fault detection scheme is designed to be robust to system parameters' uncertainties. Experimental results have attested the effectiveness of proposed fault detection scheme. 



\section{Chapter 6}

\section{Conclusions and Future Research}

This chapter aims to close the thesis by providing a brief overview of the contributions presented in this work, possible future research leads that stem from this research work, and an overview of the open challenges related to the proposed door opening method and the fault detection scheme.

\subsection{Conclusions}

The work presented in this thesis is twofold: i) the development of door opening method with mobile modular and reconfigurable robot based on the framework of the multiple working mode control. The presented door opening method solves a major problem of door opening without using complicated control algorithms or any special mechanical design. By switching between active and passive working modes of the MRR joint modules, the occurrence of large internal forces was prevented. Both simulation and experimental verification of the proposed door opening method was presented. The proposed door opening method can be implemented on any other robot that has joints capable of working in both active and passive modes. ii) the design of distributed fault detection scheme for MRRs. A distributed fault detection scheme that does not require motion states of any other modules is proposed for modular and reconfigurable robots with joint torque sensing. Unlike some previously proposed fault detection schemes, it's independent of measurements or estimations of joint accelerations. Since precise knowledge of the robot model and parameters may not be possible, the proposed fault detection scheme is designed to be robust to system parameters' uncertainties. Experimental results have manifested the effectiveness of the proposed fault detection scheme. 


\subsubsection{Strengths of the Proposed Approaches}

\section{Door Opening Method}

The task of opening a closed door with mobile MRR was studied in this thesis work. Opening a closed door have to be performed very often and is one of the major challenges for a mobile robot manipulator. It also represents a wide class of robotic operations in unstructured environments. The proposed door opening method utilizes the multiple working modes of the MRR modules where the passive working mode was cleverly employed to provide the mobile MRR with the flexibility to adapt to minor misalignments common in real-world unstructured environments. A prior knowledge about the door parameters is not required. For example, the door opening direction can be determined through differential force analysis by applying minute force at the handle, in an arbitrary direction after the door' latch has been unlocked. Then, the door opening direction is selected as the direction in which the force buildup is minimal. All other parameters including the door radius, the mobile base initial position, and the knob height with respect to the base of the MRR are estimated using the proposed door parameters estimation method. The proposed door opening method does not require any mechanical modification to the robot or its environment and avoids exploiting complicated control algorithms commonly employed to complete the door opening task with a mobile manipulator. Service robots, rescue, security and many other mobile robot manipulator applications will benefit from the proposed door opening approach.

\section{Distributed Fault Detection Method for MRR}

The proposed fault detection is modular. The fault detection for any joint does not require the motion states of the other joints, which is desirable for MRRs. In the proposed method, the dynamic properties of the upper links and joints connected to the $i$ th joint are fully reflected by the physical joint torque that is measured by the integrated joint toque sensor and the remaining coupling is the sum of multiples of lower joints motor inertias by lower joints velocities and/or accelerations. The coupling is in the actuator dynamics only and is small because of the high gear ratio. In the proposed method, this coupling was treated as modeling errors, and an adaptive update law based on the sigma-modification concepts to guarantee convergence of the estimated parameters and avoid parameter drift was designed. Most of the previously proposed fault detection schemes work only when the robot is moving freely in space, without consideration of unknown payload or contact with the environment. That is due to the fact that the filtered torque estimate is independent of the external forces applied to the robot and therefore, false alarms occur when even a small external force is applied to any part of the robot. In the method proposed in this work, exogenous force due to end-effector loads 
or impact disturbances are directly measured using the integrated joint torque sensors. In addition, joint torque measurements are used to emulate the friction dependence on payload which helps achieve small prediction error in the absence of fault.

\subsubsection{Limitations of the Proposed Approaches Door Opening Method}

The proposed method was devised for pulling doors open only. An expansion to the proposed method to allow the mobile MRR to push doors open is needed. A disadvantage associated with the proposed door opening method is that it requires a relatively large space around the door as the mobile robot has to turn to fully open the door. In addition, firm grasping was assumed at all times, however; experiments showed that there is minor relative motion between the robot end-effector and the door knob. This minor relative motion did not affect the door opening performance as the MRR robot is not required to follow a specific trajectory in task space. That is because the passive joints (Joints 1, 4, and 5) are free to move during the door opening phase and their motion is guided by the motion of the door itself.

\section{Distributed Fault Detection Method for MRR}

The detection of small magnitude faults is a major challenge for the proposed fault detection method with adaptive parameter estimation. This problem is caused by the fact that small fault effects may be compensated due to the compensation of modeling errors and parameter variations. Another limitation of the proposed fault detection scheme is that non-measurable external disturbances are not considered. The principle of the Unknown Input Observer (UIO) might be used to decouple the estimation error from the unknown inputs or disturbances. Measurement noise and dynamic modeling errors result in small prediction error as seen in the experimental result presented in chapter 5 , however, the performance of the proposed method need to be investigated in case of large amount of measurement noise is present.

\subsection{Future Work}

To carry on this research work to the next stages, efforts may be made to implement the technique of utilizing multiple working modes of the MRR joint modules on different mobile manipulator applications. One possibility of adding a degree of autonomy to the existing system would be to incorporate stereo-vision system for door localization and door knob grasping. In the future, work may also be directed to push open doors and opening of spring-loaded doors. 
The detection of small magnitude faults remains challenging for the proposed fault detection method with adaptive parameter estimation. This problem may be overcome by considering the effect of faults as a slowly varying parameter that can be estimated along with the friction parameters. If it is assumed that the system parameters and faults vary at different speeds, two filters with different gains can be designed to distinguish between incipient faults and parameters uncertainty. Future investigations would be concerned with the detection of incipient faults and residual processing schemes to detect faults while rejecting any false alarms caused by noise and spurious signals. Fault detection schemes for the mobile base would also be investigated. 


\section{Appendix 1}

\section{Hermite Cubic Spline Example}

An example demonstrating simple trajectory generation by connecting three points, $(1,1),(2,1)$, and $(3,2)$, using Hermite cubic polynomial is discussed below (only the $\mathrm{x}$-axis case is solved in detail). The following equation defines the Hermite cubic polynomial in the $x$-direction:

$$
X_{i}(t)=a_{i}(t-1)^{2}(2 t+1)+b_{i} t^{2}(3-2 t)+c_{i} t(t-1)^{2}-d_{i} t^{2}(1-t)
$$

The initial and final $x$-axis positions are " 1 " and " 3 " respectively, while the initial and final velocities are set to 0 . The boundary conditions are:

$$
X_{1}(1)=X_{2}(0)=p_{x_{2}}, \frac{d X_{1}}{d t}(1)=\frac{d X_{2}}{d t}(0), \frac{d^{2} X_{1}}{d t^{2}}(1)=\frac{d^{2} X_{2}}{d t^{2}}(0)
$$

Two segments, having four boundary conditions and four continuity conditions, are required to connect the three points. These conditions result in eight equations and eight unknowns. Rearranging those equations into a matrix format as in 4.13 yields:

$$
\left[\begin{array}{lll}
1 & 0 & 0 \\
0 & 4 & 0 \\
0 & 0 & 1
\end{array}\right]\left[\begin{array}{l}
\eta_{0} \\
\eta_{1} \\
\eta_{2}
\end{array}\right]=\left[\begin{array}{c}
v_{\text {init }}=0 \\
3\left(p_{x_{3}}-p_{x_{1}}\right)=6 \\
v_{\text {final }}=0
\end{array}\right]
$$

Solving for $\eta$ yields:

$$
\left[\begin{array}{l}
\eta_{0} \\
\eta_{1} \\
\eta_{2}
\end{array}\right]=\left[\begin{array}{c}
0 \\
1.5 \\
0
\end{array}\right]
$$




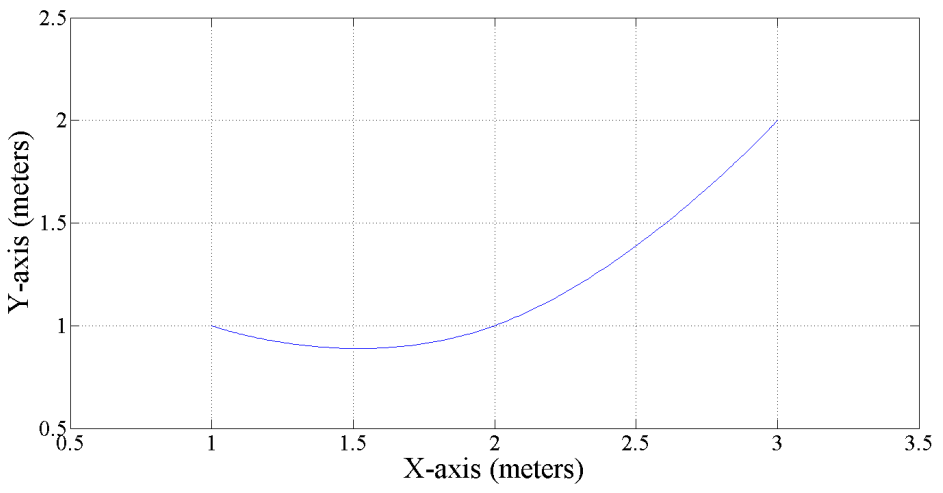

Figure 1.1: Example of two cubic splines.

The coefficients of the equations are found by the following relations:

$$
a_{i}=p_{x_{i}} ; b_{i}=p_{x_{i+1}} ; c_{i}=\eta_{i-1} ; d_{i}=\eta_{i}
$$

Therefore:

$$
\begin{aligned}
& a_{1}=1 ; \quad a_{2}=2 ; \quad b_{1}=2 ; \quad b_{2}=3 ; \\
& c_{1}=0 ; \quad c_{2}=1.5 ; \quad d_{1}=1.5 ; \quad d_{2}=0
\end{aligned}
$$

The coefficients of the y-axis is also computed using the same method:

$$
\begin{aligned}
& a_{1}=1 ; \quad a_{2}=1 ; \quad b_{1}=1 ; \quad b_{2}=2 ; \\
& c_{1}=0 ; \quad c_{2}=0.75 ; \quad d_{1}=0.75 ; \quad d_{2}=0
\end{aligned}
$$

Using these coefficients in the Hermite cubic spline equation, the path connecting the three points is shown in Fig. 1.1. 


\section{Appendix 2}

\section{Selection of the Upper Bound for the Dead-zone Residual Function}

In this Appendix, the selection of the upper bound for the dead-zone residual function in (5.18) is explained. Recall that the prediction error equation (5.16) is

$$
\mathbf{e}_{i}(t)=Y_{f i}\left(q_{i}, \dot{q}_{i}, \tau_{s i}\right) \tilde{\theta}_{i}+\zeta_{f i}
$$

Taking the vector norm of (2.1) yields:

$$
\left|\mathbf{e}_{i}(t)\right|=\left|Y_{f i}\left(q_{i}, \dot{q}_{i}, \tau_{s i}\right) \tilde{\theta}_{i}+\zeta_{f i}\right| \leq\left|Y_{f i}\left(q_{i}, \dot{q}_{i}, \tau_{s i}\right) \tilde{\theta}_{i}\right|+\left|\zeta_{f i}\right|
$$

where

$$
\begin{gathered}
Y_{f i}\left(q_{i}, \dot{q}_{i}, \tau_{s i}\right)=f(t) *\left[\begin{array}{llll}
\dot{q}_{i} & g_{l}\left(\tau_{s i}\right) \operatorname{sign}\left(\dot{q}_{i}\right) & e^{-\hat{F}_{\tau i} \dot{q}_{i}^{2}} \operatorname{sign}\left(\dot{q}_{i}\right) & -\hat{F}_{s i} \dot{q}_{i}^{2} e^{-\hat{F}_{\tau i} \dot{q}_{i}^{2}} \operatorname{sign}\left(\dot{q}_{i}\right)
\end{array}\right] \\
\tilde{\theta}_{i}=\left[\begin{array}{llll}
\hat{B}_{i}-B_{i} & \hat{F}_{c i}-F_{c i} & \hat{F}_{s i}-F_{s i} & \hat{F}_{\tau i}-F_{\tau i}
\end{array}\right]^{T}
\end{gathered}
$$

In general form and dropping the subscript $\left(q_{i}, \dot{q}_{i}, \tau_{s i}\right)$ from $Y_{f i}\left(q_{i}, \dot{q}_{i}, \tau_{s i}\right)$

$$
\begin{gathered}
Y_{f i}=\left[\begin{array}{llll}
y_{i 1} & y_{i 2} & y_{i 3} & y_{i 4}
\end{array}\right] \\
\tilde{\theta}_{i}=\left[\begin{array}{llll}
\tilde{\theta}_{i 1} & \tilde{\theta}_{i 2} & \tilde{\theta}_{i 3} & \tilde{\theta}_{i 4}
\end{array}\right]^{T} \\
Y_{f i} \tilde{\theta}_{i}=y_{i 1} \tilde{\theta}_{i 1}+y_{i 2} \tilde{\theta}_{i 2}+y_{i 3} \tilde{\theta}_{i 3}+y_{i 4} \tilde{\theta}_{i 4}
\end{gathered}
$$


Therefore

$$
\begin{aligned}
\left|Y_{f i} \tilde{\theta}_{i}\right| & =\left|y_{i 1} \tilde{\theta}_{i 1}+y_{i 2} \tilde{\theta}_{i 2}+y_{i 3} \tilde{\theta}_{i 3}+y_{i 4} \tilde{\theta}_{i 4}\right| \\
& \leq\left|\tilde{\theta}_{i 1} y_{i 1}\right|+\left|\tilde{\theta}_{i 2} y_{i 2}\right|+\left|\tilde{\theta}_{i 3} y_{i 3}\right|+\left|\tilde{\theta}_{i 4} y_{i 4}\right|
\end{aligned}
$$

If we assume that each of the friction parameters $\theta_{i}$ defined in 2.28 can be lower and upper bounded as expressed in the following inequalities

$$
\underline{\theta}_{j}<\theta_{j}<\bar{\theta}_{j}
$$

where $\theta_{j}$ denote the $j$ th component of the vector $\theta_{i}$ and $\underline{\theta}, \bar{\theta}$ denote vectors of known, constant bounds for the unknown parameters. Then the terms $\tilde{\theta}_{i j}$ will have maximum of

$$
\tilde{\theta}_{i j}=\max \left\{\left|\underline{\theta}_{i j}-\hat{\theta}_{i j}\right|,\left|\bar{\theta}_{i j}-\hat{\theta}_{i j}\right|\right\}
$$

Therefore from 2.5

$$
\left|Y_{f i} \tilde{\theta}_{i}\right| \leq \sum_{j=1}^{p}\left|Y_{f i j}\right| \max \left\{\left|\underline{\theta}_{i j}-\hat{\theta}_{i j}\right|,\left|\bar{\theta}_{i j}-\hat{\theta}_{i j}\right|\right\}
$$

where $p$ represent the number of parameters in $\theta_{i}$.

Finally, based on this derivation, $\rho_{i}(t)$ in Equation $(5.18)$ can be selected as:

$$
\rho_{i}(t)=\sum_{j=1}^{p}\left|Y_{f i j}\right| \max \left\{\left|\underline{\theta}_{i j}-\hat{\theta}_{i j}\right|,\left|\bar{\theta}_{i j}-\hat{\theta}_{i j}\right|\right\}
$$




\section{Bibliography}

Abdul, S. and G. Liu (2007, Dec.). Fault tolerant control of modular and reconfigurable robot with joint torque sensing. In Proc. IEEE Int. Conf. on Robot. and Biom., Sanya, China, pp. $1236-1241$.

Abdul, S. and G. Liu (2008, May). Decentralised fault tolerance and fault detection of modular and reconfigurable robots with joint torque sensing. In Proc. IEEE Int. Conf. on Robot. and Autom., Pasadena, CA, USA, pp. 3520-3526.

Ahmad, S. and G. Liu (2010, May). A door opening method by modular re-configurable robot with joints working on passive and active modes. In Proc. IEEE Int. Conf. Robot. Autom., Anchorage, AK, USA, pp. 1480-1485.

Ahmad, S., H. Zhang, and G. Liu (2013). Multiple working mode control of door-opening with a mobile modular and reconfigurable robot. IEEE/ASME Trans. on Mech. 18(3), $833-844$.

Basseville, M. and I. V. Nikiforov (1993). Detection of Abrupt Changes: Theory and Application. Prentice-Hall, Inc. Upper Saddle River, NJ, USA.

Bishop, C. M. (2007). Pattern Recognition and Machine Learning (1st Edition). Springer.

Biswas, G., M.-O. Cordier, J. Lunze, L. Trave-Massuyes, and M. Staroswiecki (2004). Diagnosis of complex systems: Bridging the methodologies of the FDI and DX communities. IEEE Trans. Syst. Man Cybern. Syst. Part B: Cybernetics 34(5), 2159-2162.

Blau, P. (2009). Embedding wear models into friction models. Tribol. Lett. 34, 75-79.

Bo, L. C. and D. Pavelescu (1982). The friction-speed relation and its influence on the critical velocity of Stick-slip motion. Wear 82(3), 277-289.

Brambilla, D., L. M. Capisani, A. Ferrara, and P. Pisu (2008). Fault detection for robot manipulators via second-order sliding modes. IEEE Trans. Ind. Electron. 55(11), 39543963. 
Brooks, R., L. Aryananda, A. Edsinger, P. Fitzpatrick, C. Kemp, U. M. O'Reilly, E. TorresJara, P. Varshavskaya, and J. Weber (2004). Sensing and manipulating built-for-human environments. Int. J. Hum. Robot. 1(1), 1-28.

Caccavale, F. and I. D. Walker (1997, Apr.). Observer-based fault detection for robot manipulators. In Proc. IEEE Int. Conf. on Robot. and Autom., Albuquerque, NM, USA, pp. $2881-2887$.

Chan, C., K. Cheung, Y. Wang, and W. Chan (1999, Jun.). On-line fault detection and isolation of nonlinear systems. In Proc. of the American Control Conf., San Diego, CA, USA, pp. 3980-3984.

Chung, W., C. Rhee, Y. Shim, H. Lee, and S. Park (2009). Door-opening control of a service robot using the multifingered robot hand. IEEE Trans. Ind. Electron. 56 (10), 3975-3984.

Dixon, W. E., I. D. Walker, D. M. Dawson, and J. P. Hartranft (2000). Fault detection for robot manipulators with parametric uncertainty: A prediction error based approach. IEEE Trans. Robot. Autom. 16(6), 689-699.

Dowson, D. (1998). History of Tribology. Professional Engineering Publishing, London, UK.

Dupont, P., V. Hayward, B. Armstrong, and F. Altpeter (2002). Single state elastoplastic friction models. IEEE Trans. Autom. Control 47(5), 787-792.

Eski, I., S. Erkaya, S. Savas, and S. Yildirim (2011, Feb.). Fault detection on robot manipulators using artificial neural networks. Robot Cim-Int. Manuf. 27(1), 115-123.

Frank, D. M., S. X. Ding, and B. Koppen-Selinger (2000, Jun.). Current developments in the theory of FDI. In Proc. IFAC Safeprocess, Budapest, Hungary, pp. 16-27.

Freidovich, L., A. Robertsson, A. Shiriaev, and R. Johansson (2010). Lugre-model-based friction compensation. IEEE Trans. Contr. Sys. Technol. 18(1), 194-200.

Freyermuth, B. (1991, Apr.). An approach to model-based fault diagnosis of industrial robots. In Proc. IEEE Int. Conf. on Robot. and Autom., Sacramento, CA, USA, pp. 1350-1356.

Greicius, T. (2012, Oct.). Curiosity robot geologist and chemist in one. http://www. nasa. gov/mission_pages/msl/multimedia/pia15791.html.

Gustafsson, F. (2000). Adaptive Filtering and Change Detection. Wiley.

Hahn, J. O., S. H. You, Y. M. Cho, S. Kang, and K. I. Lee (2003, Dec.). Fault diagnostics in the differential brake control system using the analytical redundancy technique. In Proc. 42nd IEEE Conf. Decis. Contr., Maui, HI, USA, pp. 2276-2281. 
Hamon, P., M. Gautier, and P. Garrec (2010, Oct.). Dynamic identification of robots with a dry friction model depending on load and velocity. In Proc. IEEE/RSJ Int. Conf. on Intell. Robot. and Syst., Taipei, Taiwan, pp. 6187-6193.

Harnoy, A., B. Friedland, and S. Cohn (2008). Modeling and measuring friction effects. IEEE Control Syst. Mag. 28(6), 82-91.

Hensen, R., M. J. G. V. de Molengraft, and M. Steinbuch (2002). Frequency domain identification of dynamic friction model parameters. IEEE Trans. Contr. Sys. Technol. 10(2), 191-196.

Hibbeler, R. C. (2007). Engineering Mechanics (11 ed.). Prentice Hall.

Hwangand, I., S. Kimand, Y. Kimand, and C. Seah (2010, May). A survey of fault detection, isolation, and reconfiguration methods. IEEE Trans. Control Syst. Technol. 18(3), 636653.

Imran, W. and F. Reza (2009). Trajectory and temporal planning of a wheeled mobile robot on an uneven surface. Robotica 27(04), 481-498.

Ioannou, P. A. and K. S. Tsakalis (1986, Nov.). A robust direct adaptive controller. IEEE Trans. Autom. Control 31(11), 1033-1043.

Isermann, R. (2006). Fault-Diagnosis Systems - An Introduction from Fault Detection to Fault Tolerance. Springer.

Izumikawa, Y., K. Yubai, and J. Hirai (2005). Fault-tolerant control system of flexible arm force sensor fault by using reaction force observer. IEEE/ASME Trans. on Mech. 10(4), 391-396.

Jacobson, B. (2003). The stribeck memorial lecture. Tribol. Int. 36(11), 781-789.

Jain, A. and C. C. Kemp (2010, May). Pulling open doors and drawers: Coordinating an omni-directional base and a compliant arm with equilibrium point control. In Proc. IEEE Int. Conf. Robot. Autom., Anchorage, Ak, USA, pp. 1807-1814.

Katsura, S., Y. Matsumotoand, and K. Ohnishi (2007). Modeling of force sensing and validation of disturbance observer for force control. IEEE Trans. Ind. Electron. 54(1), 530-538.

Kermani, M., R. Patel, and M. Moallem (2007). Friction identification and compensation in robotic manipulators. IEEE Trans. Instrum. Meas..

Kim, D., J. Kang, C. Hwang, and K. Park (2004). Mobile robot for door opening in a house. Lect. Notes. Comput. Sc. 3215(LNAI. New York: Springer-Verlag), 596-602. 
Kim, H. M., S. H. Park, and S. I. Han (2009). Precise friction control for the nonlinear friction system using the friction state observer and sliding mode control with recurrent fuzzy neural networks. Mechatronics 19(6), 805-815.

Klingbeil, E., A. Saxena, and A. Ng (2008, Jul.). Learning to open new doors. In Proc. AAAI 17th Annu. Robot Workshop Exhib., Chicago, IL, USA.

Kobayashi, S., Y. Kobayashi, Y. Yamamoto, T. Watanabe, Y. Ohtsubo, T. Inoue, M. Yasuda, and Y. Takamori (2008, Aug.). Development of a door opening system on rescue robot for search 'UMRS-2007'. In Proc. SICE Annu. Conf., Tokyo, Japan, pp. 4981-4986.

Kragic, D., L. Petersson, and H. Christenseni (2002). Visually guided manipulation tasks. Rob. Autom. Syst. 31(2-3), 193-203.

Lansdown, A. R., A. L. Price, and J. Larsen-Basse (1987). Materials to resist wear: A guide to their selection and use. J. Tribol. 109(2), 379-380.

Lawrence, P. and S. Erik (2008, Mar.). Door breaching robotic manipulator. In Proc. SPIE Defence and Security Conf., Orlando, FL, USA.

Li, W. and J. J. E. Slotine (1989, Jun.). A unified approach to compliant motion control. In Proc. of the American Control Conf., Pittsburgh, PA, USA, pp. 1944-1949.

Liu, G. (2001, May). Control of robot manipulators with consideration of actuator performance degradation and failures. In Proc. IEEE Int. Conf. on Robot. and Autom., Seoul, Korea, pp. 2566-2571.

Liu, G. (2002, Jun.). Decomposition-based friction compensation of mechanical systems. Mechatronics 12(5), 755-769.

Liu, G., S. Abdul, and A. A. Goldenberg (2008a). Distributed control of modular and reconfigurable robot with torque sensing. Robotica 26, 75-84.

Liu, G., S. Abdul, and A. A. Goldenberg (2008b). Stabilizing modular and reconfigurable robot joint by joint using torque sensing. Robotica $26(1), 75-84$.

Liu, G., A. A. Goldenberg, and Y. Zhang (1996). Uncertainty decomposition-based robust control of robot manipulators. IEEE Trans. Contr. Sys. Technol. 4(4), 384-393.

Liu, G., A. A. Goldenberg, and Y. Zhang (2004, Sep.). Precise slow motion control of a directdrive robot arm with velocity estimation and friction compensation. Mechatronics 14 (7), $821-834$.

Liu, G., X. He, J. Yuan, S. Abdul, and A. A. Goldenberg (2008, May). Development of modular and reconfigurable robot with multiple working modes. In Proc. IEEE Int. Conf. Robot. Autom., Pasadena, CA, USA, pp. 3502-3507. 
Liu, G., Y. Liu, and A. A. Goldenberg (2011). Design, analysis, and control of a springassisted modular and reconfigurable robot. IEEE/ASME Trans. on Mech. 16(4), 695-706.

Liu, X.-Q., H.-Y. Zhang, J. Liu, and J. Yang (2000). Fault detection and diagnosis of permanent-magnet dc motor based on parameter estimation and neural network. IEEE Trans. Ind. Electron. 47(5), 1021-1030.

Magill, S. (1999, July). Study of a direct measuring skin friction gage with rubber compounds for damping. Master of science, Virginia Polytechnic Institute and State University, Blacksburg, Virginia.

McIntyre, M. L., W. E. Dixon, D. M. Dawson, and I. D. Walker (2004, Apr.). Fault detection and identification for robot manipulators. In Proc. IEEE Int. Conf. on Robot. and Autom., New Orleans, LA, USA, pp. 4981-4986.

McIntyre, M. L., W. E. Dixon, D. M. Dawson, and I. D. Walker (2005). Fault identification for robot manipulators. IEEE Trans. Robot. 21(5), 1028-1034.

Mu, D.-J., H. rui Wang, X. Wang, and L. xin Wei (2004, Aug.). Observer based friction compensation in robot control. In Proc. Int. Conf. Mach. Learn. Cybern, Nice, France, pp. 401-405.

Nagatani, K. and S. Yuta (1994, Sep.). Designing a behavior to open a door and to pass through a door-way using a mobile robot equipped with a manipulator. In Proc. IEEE/RSJ Int. Conf. Intell. Robots Syst., Munich, Germany, pp. 847-853.

Nagatani, K. and S. Yuta (1995, Aug.). An experiment on opening-door-behavior by an autonomous mobile robot with a manipulator. In Proc. IEEE/RSJ Int. Conf. Intell. Robots Syst., Pittsburgh, PA, USA, pp. 45-50.

Nagatani, K. and S. Yuta (1996, Apr.). Designing strategy and implementation of mobile manipulator control system for opening door. In Proc. IEEE Int. Conf. Robot. Autom., Minneapolis, MN, USA, pp. 2828-2834.

Namvar, M. and F. Aghili (2009). Failure detection and isolation in robotic manipulators using joint torque sensors. Robotica 28(04), 1-13.

Nandi, S., H. Toliyat, and L. Xiaodong (2005). Condition monitoring and fault diagnosis of electrical motors-a review. IEEE Trans. Energy Convers. 20(4), 719-729.

Niemeyer, G. and J. J. E. Slotine (1997, Apr.). A simple strategy for opening an unknown door. In Proc. IEEE Int. Conf. Robot. Autom., Albuquerque, NM, USA, pp. 1448-1453.

Paviglianiti, G., F. Pierri, F. Caccavale, and M. Mattei (2010). Robust fault detection and isolation for proprioceptive sensors of robot manipulators. Mechatronics 20(1), 162-170. 
Petersson, L., D. Austin, and D. Kragic (2000, Nov.). High-level control of a mobile manipulator for door opening. In Proc. IEEE/RSJ Int. Conf. Intell. Robots Syst., Takamatsu, Japan, pp. 2333-2338.

Prassler, E. (2013, Jan.). DESIRE: Germany service robotics initiative. http://www. service-robotik-initiative.de/uebersicht/uebersicht/?lang=en.

Ray, L. and J. Remine (1998, Jun.). Machine friction estimation for modeling, diagnostics, and control. In Proc. of the American Control Conf., pp. 2737-2741.

Ryew, S. and H. Choi (2001). Double active universal joint (DAUJ): Robotic joint mechanism for human-like motions. IEEE Trans. Robot. Autom. 17(3), 290-300.

Schneider, H. and P. M. Frank (1996). Observer-based supervision and fault detection in robots using nonlinear and fuzzy logic residual evaluation. IEEE Trans. Control Syst. Technol. $4(3), 274-282$.

Shin, J.-H. and J.-J. Lee (1999, May). Fault detection and robust fault recovery control for robot manipulators with actuator failures. In Proc. IEEE Int. Conf. on Robot. and Autom., Detroit, MI, USA, pp. 861-866.

Söderström, T. (1981). Identification of stochastic linear systems in presence of input noise. Automatica 17(5), 713-725.

Strang, G. (1986). Introduction to Applied Mathematics. Wellesley-Cambridge Press, Wellesley, MA.

Tien, L. L., A. Albu-Schaffer, A. D. Luca, and G. Hirzinger (2008, Sep.). Friction observer and compensation for control of robots with joint torque measurement. In Proc. IEEE/RSJ Int. Conf. on Intel. Robot. and Sys., Nice, France, pp. 3789-3795.

Vemuri, T. and M. M. Polycarpou (2004). A methodology for fault diagnosis in robotic systems using neural networks. Robotica 22(04), 419-438.

Venkatasubramanian, V., R. Rengaswamy, and S. N. Kavuri (2003). A review of process fault detection and diagnosis: Part II: Qualitative models and search strategies. Comput. Chem. Eng. 27(3), 313-326.

Waarsing, B. J. W., M. Nuttin, and H. V. Brussel (2003, Mar.). Behaviour-based mobile manipulation: The opening of a door. In 1stInt. Workshop on Advances in Service Robotics, Bardolino, Italy, pp. 170-175.

Wang, M., S. Ma, B. Li, and Y. Wang (2009). Reconfiguration of a group of wheelmanipulator robots based on MSV and CSM. IEEE/ASME Trans. on Mech. 14(2), 229239. 
Woydt, M. and R. Wäsche (2010). The history of the stribeck curve and ball bearing steels: The role of Adolf Martens. Wear 268(11-12), 1542-1546.

Yongli, Z., H. Limin, and L. Jinling (2006). Bayesian networks-based approach for power systems fault diagnosis. Power Delivery, IEEE Transactions on 21(2), 634-639.

Yuan, J., G. Liu, and B. Wu (2011, Oct.). Power efficiency estimation-based health monitoring and fault detection of modular and reconfigurable robot. IEEE Trans. Ind. Electron. 58(10), 4880-4887.

Zeigler, B. (1992). Systems formulation of a theory of diagnosis from first principles. IEEE Trans. Rel. $41(1), 38-48$.

Zhang, X. (2011, May). Sensor bias fault detection and isolation in a class of nonlinear uncertain systems using adaptive estimation. IEEE Trans. Autom. Control 56(5), 12201226.

Zhong, B., L. Sun, L. Chen, and Z. Wang (2011, Aug.). The dynamics study of the stick-slip driving system based on lugre dynamic friction model. In Proc. Int. Conf. Mech. Autom., pp. $584-589$.

Zhu, W.-H., E. Dupuis, and M. Doyon (2007, Mar.). Adaptive control of harmonic drives. J. Dyn. Sys., Meas., Control 129(2), 182-193. 

Chapter 2 
